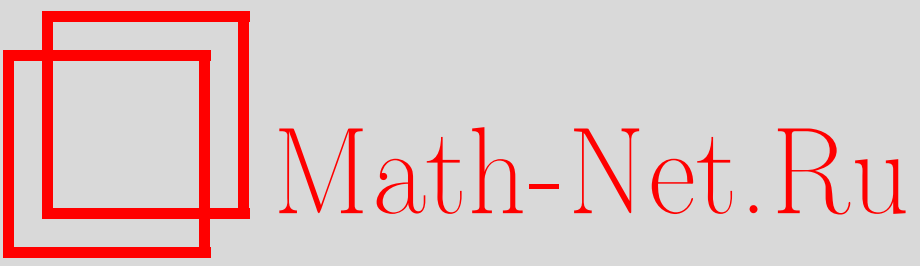

А. И. Дегтярев, В. М. Харламов, Топологические свойства вещественных алгебраических многообразий: du côté de chez Rokhlin, УМH, 2000, том 55, выпуск 4, 129-212

DOI: https://doi.org/10.4213/rm315

Использование Общероссийского математического портала Math-Net.Ru подразумевает, что вы прочитали и согласны с пользовательским соглашением

http://www . mathnet.ru/rus/agreement

Параметры загрузки:

IP: 54.174 .149 .18

26 апреля 2023 г., 18:03:10 


\section{ТОПОЛОГИЧЕСКИЕ СВОЙСТВА ВЕЩЕСТВЕННЫХ АЛГЕБРАИЧЕСКИХ МНОГООБРАЗИЙ: DU CÔTÉ DE CHEZ ROKHLIN}

А. И. ДЕГТЯРЕВ, В.М. ХАРЛАМов

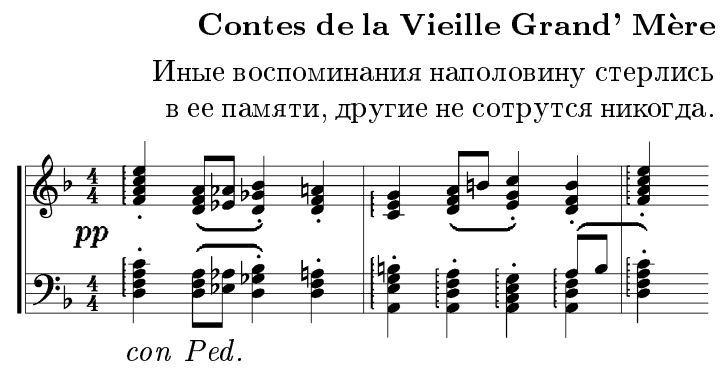

С. С. ПРОКОФЬЕВ. Соч. 31 (1918)

Предлагаемый обзор охватывает резултаты, достигнутые в топологии вещественных алгебраических многообразий в направлении исследований, развернутых в начале 70-х годов В.И. Арнольдом и В.А. Рохлиным. Мы сделали попытку систематизировать основные достижения в этой области. Представив общие технические средства и результаты, мы уделяем особое внимание поверхностям и кривым на поверхностях.

Библиографоия: 131 название.

\section{СОДЕРЖАНИЕ}

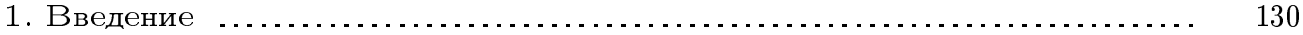

2. Технические средства и общие результаты $\ldots \ldots \ldots \ldots \ldots \ldots \ldots \ldots \ldots \ldots . . \ldots \ldots \ldots$

3. Поверхности ..................................................... 150

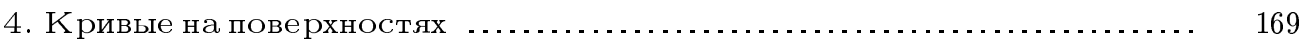

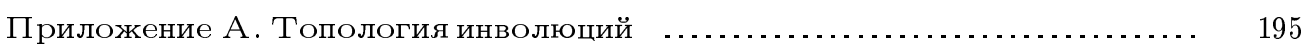

Приложение В. Целочисленные решетки и квадратичные формы $\ldots . . . . .203$

Приложение С. Форма Рохлина-Гийю-Марена ......................... 206

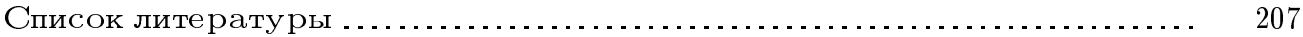




\section{1. Введение}

Поворотный этап. Интерес, проявленный Владимиром Абрамовичем Рохлиным к топологии вещественных алгебраических многообразий, был в значительной степени обусловлен результатами, полученными в конце 60-х гг. Д. А. Гудковым [45], и последовавшей за ними работой В. И. Арнольда [4], которые внесли значительньй вклад в решение 16-й проблемы Гильберта. Гудков опроверг одну из гипотез Гильберта о расположении овалов - двусторонних компонент - плоских $M$-секстик (т.е. секстик с максимальным числом овалов). Он исправил гипотезу, доказал ее для степени 6 и предложил в [40] распространение своего результата на $M$-кривые любой четной степени в качестве новой гипотезы. (Напомним, что $M$-кривая рода $g$ - это кривая с максимальным числом связных компонент своей вещественной части; согласно неравенству Харнака это максимальное число есть $M=g+1$.)

Чтобы сформулировать гипотезу Гудкова, напомним, что овал плоской кривой называется четным (нечетным), если он лежит внутри четного (соответственно нечетного) числа других овалов. Число четных (нечетных) овалов обозначается через $p$ (соответственно через $n$ ). В этих обозначениях гипотеза Гудкова утверждает, что $p-n=k^{2} \bmod 8$ для любой $M$-кривой степени $2 k$.

В замечательной работе В. И. Арнольда [4] была найдена связь вешественных плоских кривых с топологией четырехмерных многообразий и арифметикой целочисленных квадратичных форм и, среди других результатов, доказана ослабленная гипотеза Гудкова $\left(p-n=k^{2} \bmod 4\right)$. События после этого стали быстро развиваться. Во-первых, Рохлин предложил доказательство гипотезы Гудкова, основанное на своей формуле, связывающей сигнатуру четырехмерного многообразия с Arf-инвариантом характеристической поверхности (см. приложение C). Затем после того, как В.М. Харламов [55] обобщил результат Арнольда на случай поверхностей, Рохлин [100] нашел другое доказательство, не использующее никакой специфики четырехмерной топологии, и распространил этот результат на многообразия любой размерности. На этом этап революционных достижений, начатьй Арнольдом, был завершен, и началось систематическое изучение предмета. (Другие детали и краткий обзор дальнейшей истории вопроса имеются в [41], [126] и [6].)

Наследие Рохлина. Рохлин опубликовал шесть работ по топологии вещественных алгебраических многообразий (см. [97]-[102]). Это число невелико, но каждая из этих работ дала начало целому новому направлению в этой области. (Единственным исключением, возможно, является короткая заметка [101], которая распространяет ряд приложений некоторых предыдущих результатов с полных пересечений на произвольные вещественные алгебраические многообразия.) В этом обзоре мы более или менее подробно обсуждаем все эти работы, кроме [102], в которой для изучения алгебраических кривых используются неалгебраические накрытия; другие примеры такого использования см. в [35].

Первая работа Рохлина [97] содержит ошибку в доказательстве гипотезы Гудкова. Однако предложенньй подход, а именно, использование характеристических поверхностей в четырехмерном многообразии для вычисления его сигнатуры $\bmod 16$, стал

\footnotetext{
Заглавие имитирует трудно переводимое название романа Марселя Пруста, ср. "По направлению к Свану" и "Swan's way". (См. также аналогичную игру слов в кн. B поискаx утраченной топологии. Под ред. Л. Гийу и А. Марена. М.: Мир, 1989.)
} 
мошным методом изучения вешественных алгебраических кривых. Он был использован Мареном, которьй вместе с Гийю (см. [46]) распространил сигнатурную формулу Рохлина на неориентируемые характеристические поверхности и тем самым исправил указанную ошибку. (Формула Рохлина-Гийю-Марена и связанная с ней квадратичная форма рассматриваются в приложении С.)

Другим фундаментальным результатом, которьй трудно переоценить, является формула Рохлина для комплексных ориентаций. Понятие комплексной ориентации разбиваюшей вешественной кривой (см. ниже), как, впрочем, и сама формула Рохлина и ее доказательство, представляются невероятно прозрачными. Однако эта формула устанавливает, например, справедливость двух гипотез Гильберта об 11 овалах плоских секстик, которые сам Гильберт пытался доказать чрезвычайно сложньм путем и включил их в свой знаменитый список проблем (в качестве 16-й проблемы). В настояшее время формула Рохлина является главным средством в изучении топологии вещественных кривых на поверхностях. Она относится к тем немногим свойствам кривых, которые до сих пор не имеют удовлетворительного обобщения на многообразия старших размерностей. (Фактически, даже для кривых на поверхностях некоторые фундаментальные вопросы до сих пор не вполне выяснены, cp. 4.2.)

Стоит упомянуть, что первым начал изучать разбивающие кривые $\Phi$. Клейн. Он открыл некоторые их замечательные свойства и указал, как в случае таких кривых можно улучшить и обобшить неравенство Харнака для числа компонент связности кривой. Однако он не заметил комплексных ориентаций.

Формула Рохлина для комплексных ориентаций и 16-я проблема Гильберта. Неприводимая над $\mathbb{R}$ неособая вешественная кривая $A$ (т.е. либо неприводимая неособая комплексная кривая с антиголоморфной инволюцией conj: $A \rightarrow A$, либо неособая комплексная кривая, состоящая из двух неприводимых компонент, переставляемых антиголоморфной инволюцией) назьвается разбивающей или кривой mипа I, если ее вешественная часть $\mathbb{R} A=$ Fix conj разбивает $A$ на две половины две связные поверхности $A_{+}$и $A_{-}$с обшим краем $\mathbb{R} A$. (Заметим, что если кривая неприводима над $\mathbb{R}$, но приводима над $\mathbb{C}$, то она разбиваюшая.) Комплексное сопряжение conj: $A \rightarrow A$ переставляет поверхности $A_{ \pm}$, стандартные ориентации которых индуцируют две противоположные ориентации на $\mathbb{R} A$, называемые комплексными ориентачиями. Приводимая или особая кривая называется разбивающей, если нормализация каждой неприводимой над $\mathbb{R}$ компоненты этой кривой принадлежит типу I. Ясно, что кривая $A$ является разбивающей тогда и только тогда, когда фактор-пространство $A /$ conj есть ориентируемая поверхность, и если это условие выполнено, комплексные ориентации индуцируются ориентацией края поверхности $A /$ conj. Основньм примером служат $M$-кривые, которые все являются разбиваюшими.

Пусть $A$ - неособая разбивающая плоская кривая. Говорят, что два ее овала образуют инбективную пару, если они ограничивают кольцо в $\mathbb{R P}^{2}$. Инъективная пара назьвается положительной, если комплексные ориентации овалов индуцируются некоторой ориентацией кольца, и отрицательной в противном случае. Обозначим через $\Pi^{+}$и $\Pi^{-}$числа положительных и соответственно отрицательных инъективных пар и положим $\Pi=\Pi^{+}+\Pi^{-}$. 
1.1. ФормУлА РохлИна. Для неособой разбивающей плоской кривой четной степени $2 k \quad c \quad l$ вещественными компонентами справедливо равенство $2\left(\Pi^{+}-\Pi^{-}\right)=l-k^{2}$.

Формула Рохлина имеет многочисленные применения. Например, ее непосредственными следствиями являются сравнения Арнольда и Слепяна: для разбивающей кривой степени $2 k$ согласно Арнольду $p-n=k^{2} \bmod 4$, а если непосредственно внутри каждого нечетного овала лежит нечетное число четных овалов (т.е. компонента дополнения кривой, ограниченная снаружи этим нечетным овалом, имеет четную эйлерову характеристику), то согласно Слепяну $p-n=k^{2} \bmod 8$. Другим следствием формулы Рохлина является то, что $\Pi \geqslant\left|l-k^{2}\right|$ и $l \geqslant k$ для любой разбивающей кривой степени $2 k$. Последнее неравенство вместе со сравнением Клейна $l=k \bmod 2$, которое также следует из формулы Рохлина, дает полньй набор ограничений на число компонент неособой разбивающей плоской кривой степени $2 k$; для кривых степени $2 k-1$ из соответствующего варианта формулы Рохлина (формулы Рохлина-Мишачева, см., например, [99]) следует неравенство $l \geqslant k$ и аналог предыдушего утверждения. ${ }^{1}$

Для заданной разбивающей кривой $A$ ее вещественная схема (т.е. топологический тип пары $\left.\left(\mathbb{R P}^{2}, \mathbb{R} A\right)\right)$ и комплексная ориентация определяют $A \cup \mathbb{R} \mathbb{P}^{2}$ с точностью до гомеоморфизма. (Если кривая не разбивающая, $A \cup \mathbb{R P}^{2}$ определяется вещественной схемой кривой $A$.) Одна из связанных с этим проблем, предложенная Рохлиным, состоит в изучении комплексных ориентаций, возникающих на кривых данной степени; она занимает промежуточное положение между более тонким вопросом об эквивариантной топологии пары $\left(\mathbb{P}^{2}, A\right)$ и более грубым - о топологии пары $\left(\mathbb{R} \mathbb{P}^{2}, \mathbb{R} A\right)$ (исходной формулировкой 16-й проблемы Гильберта). Стоит отметить и две другие связанные с этим проблемы: изучение фундаментальной группы $\pi_{1}\left(\mathbb{P}^{2} \backslash\left(\mathbb{R} \mathbb{P}^{2} \cup \mathbb{R} A\right)\right)$ и изучение топологии пространства $\left(\mathbb{R} \mathbb{P}^{2} \cup A\right) \subset \mathbb{P}^{2} /$ conj $=S^{2}$.

Школа Рохлина. Влияние Рохлина на топологию вещественных алгебраических многообразий было гораздо шире его собственных результатов. В этой области он руководил работой нескольких своих учеников (Виро, Звонилова, Мишачева, Слепяна, Фидлера, Финашина, Харламова, Чепонкуса) и шедро делился своими идеями и обширньми знаниями. К сожалению, Владимир Абрамович не смог осуществить все свои планы: безвременная кончина прервала его работу над целым рядом проектов.

Еше в середине 70-х гг. у Рохлина была идея написать два подробных обзора результатов, полученных к тому времени: один - по вещественным плоским кривым, другой - по многообразиям старших размерностей. В самом начале к этой работе был привлечен В. М. Харламов, а позже - О. Я. Виро. Были разработаны детальные планы, Рохлин написал краткий конспект первых глав книги о плоских кривых, а Харламов - первых глав второй книги. Рохлин планировал прочитать соответствующие лекционные курсы в Ленинградском государственном университете; к сожалению, он был вынужден уйти на пенсию, и курс о плоских кривых был впервые прочитан Виро (в сокращенном варианте), а затем Виро и Харламовым. Виро и Харламов продолжили работу над первым проектом после кончины Рохлина. Несколько раз работа прерывалась по различньм политическим и географическим причинам. Однако черновик книги был написан и значительная его часть была отредактирована. Несколько

\footnotetext{
${ }^{1}$ Как мы узнали от А. Габара (A. Gabard), вопрос о числе компонент разбивающей кривой данной степени был впервые поставлен $\Phi$. Клейном.
} 
студентов использовали предварительный вариант книги в качестве введения в предмет. К сожалению, эта книга, скорее всего, никогда не появится (отчасти потому, что развитие этой области происходит быстрее написания текста). Введение в обзоре Виро [117] может дать представление о первых главах книги. (Обзор Виро, посвященньй, в основном, другим вопросам в этой области - вопросам построения, шире охватывает основные понятия и результаты, в то время как книга содержит тонкости, уместные лишш в учебном тексте.) Настоящий обзор отражает идеи второго проекта; изначально он возник как приложение к книге о кривых (что, естественно, также оказало влияние на его содержание: значительная часть утверждений в 4.6 и 4.7 является непосредственным обобщением соответствующих результатов для кривых, имеющихся в книге).

Что есть и чего нет в обзоре. Мы не пытаемся полностью охватить все известные результаты в топологии вешественных алгебраических многообразий: предмет настолько развит и разнообразен, что, по-видимому, невозможно изложить его в пределах разумного объема. Вместо этого мы стараемся отобрать результаты, теснее связанные с тем, что открыл и изучил в этой области Рохлин и его последователи (конечно, даже этот выбор не является исчерпьваюшим и, в основном, определяется нашим личным вкусом), и показать, что могут дать предложенные подходы и как работает развитая техника. Большинство доказательств либо опушены, либо лишш намечены. Ссылки также неполны; во многих случаях мы ссылаемся на те работы и учебники, в которых лучше изложены соответствующие идеи и доказательства, а не на оригинальные работы.

Исторически, в топологии вешественных алгебраических многообразий особое внимание уделялось случаю плоских кривых; мы адресуем читателя к прекрасным обзорам [41], [126], [117] и сосредоточиваемся на результатах для старших размерностей. Однако в конце мы возврашаемся к поверхностям и кривым на поверхностях и обсуждаем некоторые последние достижения, особенно те, которые связаны с комплексными ориентациями. (Заметим, что топологические свойства абстрактных, не вложенных вешественных кривых просты и были известны еше во времена $\Phi$. Клейна, см., например, [99], [87].) Отдельная тема - трехмерные вешественные многообразия. Долгое время они оставались за рамками предмета; недавно благодаря Я. Коллару положение начало меняться, и в настоящее время трехмерные многообразия заслуживают отдельного разговора.

Большинство известных результатов для плоских кривых следует из соответствующих результатов для некоторых вспомогательных поверхностей; более того, во многих случаях общие формулировки для старших размерностей более удобны, так как дают лучшее понимание связи между топологией вешественной части многообразия и его комплексификацией. Однако имееется несколько исключений, которые до сих пор не распространены на старшие размерности; наиболее замечательными из них являются вьшеупомянутая формула Рохлина для комплексных ориентаций и, среди недавних достижений, результаты С. Ю. Оревкова, основанные на систематическом изучении пучков прямых. (Результаты Оревкова, за исключением его формулы комплексных ориентаций 4.5.1, относятся к кривым малых степеней; их обшее понимание и связь с другими подходами до сих пор не вполне выяснены даже для плоских кривых.)

Топология вещественных алгебраических многообразий имеет два направления развития: запреты и построения. В настоящей работе мы полностью игнорируем 
последнее направление (обзор его методов и результатов см., например, в [117], [52], [96]) и сосредоточиваемся на результатах, относяшихся к запретам, т.е. ограничениям на топологию множества вещественных точек вешественного алгебраического многообразия, накладываемым топологией множества его комплексных точек. Последняя обычно считается известной; типичньй пример - изучение алгебраических многообразий в заданном комплексном деформационном семействе.

Перечислим несколько других связанных с этой областью важных тем, развиваюшихся в настояшее время, но не затрагиваемых в обзоре: специальные многочлены, малочлены, сложность вычислений, особенности и особые многообразия, аппроксимации, метрические свойства, принцип Акса, торические многообразия, алгебраические циклы, пространства модулей, минимальные модели, связи с симплектической геометрией.

Хотя наш основной предмет исследования - вешественные алгебраические многообразия, многие результаты справедливы для более широких категорий объектов. В частности, вместо алгебраического многообразия, определенного над $\mathbb{R}$, мы часто рассматриваем замкнутое комплексно-аналитическое многообразие с действующей на нем антиголоморфной инволюцией. Во многих случаях комплексная структура может быть заменена не обязательно интегрируемой почти комплексной. (Наиболее интригуюшее исключение - обобщенное неравенство Комессатти-Петровского-Олейник 2.4.1, которое имеет топологическое доказательство для поверхностей, в то время как известное доказательство для многообразий старших размерностей использует интегрируемость комплексной структуры и, более того, кэлеровость.) Более того, многие запреты являются по своей природе топологическими и, следовательно, справедливы для произвольных гладких многообразий с инволющией; иногда приходится дополнительно предполагать, что множество неподвижных точек инволюции локально устроено как множество вещественных точек вещественного алгебраического многообразия, т.е. его кокасательное и нормальное расслоения антиизоморфны. Указанные явления не очень удивляют, если учесть современные взаимосвязи между дифференциальной и алгебраической геометриями. Кроме того, можно обратить внимание на единство комплексной и вешественной алгебраических геометрий, основанное, например, на соответствии $X \mapsto X \times \bar{X}$ (где $\bar{X}$ - многообразие с комплексно сопряженной структурой) или $X \mapsto X \sqcup \bar{X}$; в последнем случае существует биекция между вешественными структурами $f: X \rightarrow \bar{X}$ и инволютивными автоморфизмами многообразия $X \sqcup \bar{X}$, имеющими вид $f \sqcup \bar{f}$.

Обозначения. Вещественная структура на комплексно-аналитическом многообразии $X$ есть антиголоморфная инволюция. (В случае алгебраических многообразий, определенных над $\mathbb{R}$, это инволюция Галуа.) Обычно мы обозначаем ее через conj: $X \rightarrow X$. Множество неподвижных точек вешественной структуры называется вещественной частью многообразия и обозначается через $\mathbb{R} X$. Фактор-пространство $X /$ conj обычно обозначается через $X^{\prime}$.

Как было упомянуто выше, многие результаты имеют топологическую природу и потому справедливы для более широких классов многообразий. Несмотря на то, что довольно трудно соединить все необходимые условия в одну формулировку, в большинстве случаев следующее определение очень удобно: гибким вещественным. многообразием называется замкнутое гладкое многообразие $X$ вместе с гладкой инволюцией conj: $X \rightarrow X$ и (не обязательно интегрируемой) почти комплексной струк- 
турой $J$ в окрестности вещественной части Fix conj такими, что инволюция соnј антиголоморфна относительно $J$. (В случае, если речь идет о применениях теоремы Безу, полезно добавить симплектическую структуру, согласованную с conj и $J$.)

Замкнутое комплексное (не обязательно неприводимое или приведенное) подмногообразие $A \subset X$ называется вещественны.м, если оно сопј-инвариантно. Чтобы не путать conj-инвариантные комплексные подмногообразия с вещественными подмногообразиями в обычном смысле, мы иногда называем первые вещественнылми гладкими ииклами (или вещественными гладкими дивизорами, если их комплексная коразмерность равна 1). Кривой в комплексном многообразии $X$ называется приведенньй эффективньй цикл (дивизор, если $X$ есть поверхность).

В алгебраической геометрии многие понятия имеют различньй смысл над $\mathbb{C}$ и над $\mathbb{R}$; для обозначения вешественного варианта мы используем префикс $\mathbb{R}$. Так, подмногообразие $A$ называется $\mathbb{R}$ - неприводимымм, если оно либо неприводимо над $\mathbb{C}$, либо имеет две компоненты, переставляемые инволюцией соnj; $\mathbb{R}$-компонентой подмногообразия $A$ назьвается либо сопј-инвариантная компонента, либо пара компонент, переставляемых инволюцией соnj. (Осознавая неуклюжесть этой терминологии, мы используем ее, не найдя лучшей. Термин "вещественньй" может вызвать путаницу этих понятий с их топологическими омонимами, а выражение “над $\mathbb{R}$ ” не всегда приемлемо грамматически или, того хуже, семантически, поскольку, вообще говоря, нашш многообразия не являются алгебраическими многообразиями, определенными над $\mathbb{R}$.)

На комплексном многообразии $X$ с вещественной структурой для линейной системы $|D|$ (см. 2.1) мы обозначаем через $\mathbb{R}|D|$ ее вешественную часть, которая состоит из вешественных дивизоров, линейно эквивалентных дивизору $D$, а через $\Delta_{|D|}$ и $\mathbb{R} \Delta_{|D|}$ - дискриминант системы $|D|$ и соответственно его вешественную часть. Если же $X=\mathbb{P}^{n}$, мы используем более употребительные обозначения $\mathscr{C}_{q}, \Delta_{q}$ для пространства гиперповерхностей степени $q$ и соответственно его дискриминанта, а также $\mathbb{R} \mathscr{C}_{q}$, $\mathbb{R} \Delta_{q}$ для их вещественных частей.

Мы пользуемся стандартными скобочными обозначениями для кодировки частично упорядоченных множеств. Типичная ситуация - кодировка множества овалов вещественной кривой на вещественной поверхности. Для каждого класса кривых используемьй частичный порядок явно указьвается; например, для двух овалов $C_{1}, C_{2}$ плоской кривой по определению $C_{1} \succ C_{2}$, если $C_{2}$ лежит в круге, ограниченном овалом $C_{1}$.

Все группы гомологий и когомологий имеют $\mathbb{Z}_{2}$-коэффициенты, если последние не указаны явно. Через $b_{i}(\cdot)$ и $\beta_{i}(\cdot)$ мы обозначаем числа Бетти над $\mathbb{Q}$ и над $\mathbb{Z}_{2}$, а через $b_{*}(\cdot)$ и $\beta_{*}(\cdot)$ - соответствующие тотальные числа Бетти. Для топологического пространства $X$ с инволюцией $c$ через $\left({ }^{r} E_{*}(X),{ }^{r} d_{*}\right)$ (или просто $\left.\left({ }^{r} E_{*},{ }^{r} d_{*}\right)\right)$ мы обозначаем спектральную последовательность Калинина, через bv * - гомоморфизмы Виро и через $\mathscr{F}^{*}(X)=\mathscr{F}^{*}$ - фильтрацию Калинина на $H_{*}($ Fix $c)$ (см. А.2).

Для многообразия $X$ через $w_{i}=w_{i}(X)$ обозначаются его классы Штифеля-Уитни, через $u_{i}=u_{i}(X)$ - его классы Ву, а если $X$ - комплексное многообразие, через $c_{i}=$ $c_{i}(X)$ - его классы Черна. Если $X$ замкнуто, через $\mathrm{D}=\mathrm{D}_{X}: H^{*}(X) \rightarrow H_{*}(X)$ обозначается изоморфизм двойственности Пуанкаре; при этом для гомологических классов, двойственных по Пуанкаре характеристическим классам, часто используются те же обозначения $w_{i}, u_{i}, c_{i}$. 
Благодарности. Мы признательны нашим многочисленным коллегам, с которыми мы совместно работали в этой области и которые делились с нами своими знаниями. Среди тех, кто во время нашей работы над обзором помогал нам выяснить некоторые детали или сообшал о своих неопубликованных результатах, - Б. Шевалье, С. М. Финашин, И. В. Итенберг, А. Марен, С. Ю. Оревков, О. Я. Виро, Ж.-И. Вельшингер. Наша особая признательность - А. М. Вершику, который вдохновил нас написать этот обзор.

\section{2. Технические средства и общие результаты}

2.1. Дивизоры и линейная эквивалентность. Напомним, что дивизором на неособом компактном комплексном многообразии $X$ назьвается формальная конечная линейная комбинация $A=\sum m_{i} D_{i}$ с $m_{i} \in \mathbb{Z}$, в которой $D_{i}$-неприводимые (возможно, особые) компактные подмногообразия многообразия $X$, имеющие коразмерность 1 . Если все $m_{i} \geqslant 0$, дивизор $A$ назьвается әффективным. Дивизоры образуют группу относительно формального сложения линейных комбинаций. Два дивизора $D_{1}, D_{2}$ назьваются линейно әквивалентными, $D_{1} \sim D_{2}$, если их разность есть главныи $\partial и в и з о р$, т.е. если сушествует мероморфная функция на $X$, нули и полюсы которой являются компонентами дивизора $D_{1}-D_{2}$ с учетом их кратностей. Все эффективные дивизоры, линейно эквивалентные данному дивизору $D$, образуют проективное пространство; оно называется линейной системой и обозначается через $|D|$. Ясно, что необходимьм условием линейной эквивалентности дивизоров является реализуемость ими одного и того же класса в $H^{2}(X ; \mathbb{Z})$; если $X$ односвязно (или, более общо, $H^{1}(X ; \mathbb{Z})=0$ ), это условие также и достаточно (см., например, [49] или [47]).

Дивизор называется очень обильным, если его можно представить в виде гиперплоского сечения при некотором вложении многообразия $X$ в проективное пространство. Дивизор $D$ называется обильным. если некоторое его положительное кратное $m D$ является очень обильным. (Некоторые полезные критерии обильности можно найти в [47].)

Если $A$ - вешественшый дивизор на вешественном многообразии ( $X$, conj), класс дивизора $A$ в $H^{2}(X ; \mathbb{Z})$ является косоинвариантным относительно соnj* . Если $H^{1}(X)=0$, каждьй дивизор, класс которого косоинвариантен относительно соnj*, линейно эквивалентен вешественному. Вешественные дивизоры, эквивалентные над $\mathbb{C}$, эквивалентны над $\mathbb{R}$, если $\mathbb{R} X \neq \varnothing$.

2.1.1. ТЕОРЕМА ЛЕФШЕЦА О ГИПЕРПЛОСКОМ СЕЧЕНИИ (см., например, [86]). Если $A$ - обильный дивизор на неособом компактном комплексном $n$-мерном многообразии $X$, то $X \backslash A$ имеет гомотопический тип п-мерного клеточного пространства. В частности, $H_{r}(X, A ; G)=H^{r}(X, A ; G)=0$ для любой абелевой группь $G$ и любого $r<n$.

2.2. Двулистные накрытия. Двулистные накрытия играют особую роль в вешественной алгебраической геометрии: расположение подмногообразия $A \subset X$ в большой степени отражается на топологических свойствах двулистного накрытия многообразия $X$, разветвленного над $A$ (согласно принципу В. И. Арнольда понятие двулистного накрытия является комплексификацией понятия многообразия с краем; по утверждению Арнольда именно благодаря этому наблюдению он нашел доказательство ослабленного сравнения Гудкова и других замечательных результатов ра- 
боты [2]). Таким образом, как только получен некоторьй результат для абсолютного случая, его можно применить к разветвленным двулистньм накрытиям и тем самьм получить некоторые запреты в относительном случае на расположение подмногообразия.

Дивизор $A \subset X$ называется четным, если фундаментальный класс $[A]$ равен нулю в $H_{2 n-2}(X)$, где $n=\operatorname{dim} X$. В этом случае сушествует двулистное накрьваюшее $Y$ многообразия $X$ с ветвлением над $A$, причем $Y$ также является комплексньм многообразием, неособьм, если $A$ неособо. Ясно, что с точностью до изоморфизма накрытие определяется классом $\omega \in H^{1}(X \backslash A)$, образ которого при $H^{1}(X \backslash A) \rightarrow$ $H_{2 n-1}(X, A) \rightarrow H_{2 n-2}(A)$ равен $[A]$. С другой стороны, равенство нулю гомологического класса равносильно тому, что $A \sim 2 E$ для некоторого дивизора $E$ на $X$, и классы изоморфных двулистных накрытий находятся в каноническом взаимно однозначном соответствии с такими классами $E \in \operatorname{Pic} X$, что $2 E \sim A$, см. [48]. Для конкретно выбранного накрытия мы используем обозначение $Y(E)$.

Следующее утверждение сразу следует из 2.1.1:

2.2.1. ТЕОРЕМА ЛЕФШЕЦА ДЛЯ ДВУЛИСТНЫХ НАКРЫТИЙ. Пусть $A-$ четный обильный дивизор на неособом компактном комплексном многообразии $X, u$ пусть $Y$ - двулистное разветвленное накрывающее многообразия $X$ с ветвлением над А. Тогда для любой абелевой группы $G$

$$
H_{r}(Y, A ; G)=H^{r}(Y, A ; G)=0, \quad \text { əде } \quad r<n=\operatorname{dim} X
$$

2.2.2. С ЛЕДСТВИЕ ИЗ 2.1.1 и 2.2.1. Пусть $A \subset X$ и $Y$ - maкuе жсе, как в 2.2.1, и $p: Y \rightarrow X-$ проекиия накрытия. Тогда для любой абелевой группы $G$ индуцированное отображсение $p_{*}: H_{r}(Y ; G) \rightarrow H_{r}(X ; G)$ есть изоморфизм для $r<n u$ эпиморфизм для $r=n$; индуцированное отображсение $p^{*}: H^{r}(X ; G) \rightarrow H^{r}(Y ; G)$ есть изоморфизм для $r<n$ и мономорфизм для $r=n$. Более того, если $G-$ поле $c$ char $G \neq 2$, то $p_{*}$ индуцирует изоморфизм межсду $H_{*}(X ; G)$ и подгруппой $H_{*}^{+}(Y ; G)$ классов, инвариантных относительно автоморфизма накрытия (соответственно $p^{*}$ индуиирует изоморфизм межсду $H^{*}(X ; G)$ и $H_{+}^{*}(Y ; G)$ ).

Заметим, что 2.2.1 и 2.2.2 остаются справедливыми, если $A$ и, следовательно, $Y$ имеют особенности. Если дивизор $A$ гладкий, но не является четным, можно попытаться найти другой гладкий дивизор $H$ на $X$, пересекающий $A$ трансверсально и такой, что $[H]+[A]=0$ в $H_{2 n-2}(X)$. Тогда существует двулистное накрывающее $Y_{H}$ многообразия $X$ с ветвлением над $H \cup A$, и можно изучать его раздутие $\widetilde{Y}_{H}$ с центpoм $S=H \cap A$. (Типичньй пример - когда $X \subset \mathbb{P}^{N}$ и $A$ высекается на $X$ гиперплоскостью нечетной степени: взяв в качестве $H$ общее гиперплоское сечение, получим информацию об аффинной части пары $(X, A)$.) Результат будет тем же, если раздуть $S$ на $X$, получив неособое многообразие $\widetilde{X}$, и взять в качестве $\widetilde{Y}_{H}$ двулистное разветвленное накрьвающее многообразия $\widetilde{X}$ с ветвлением над собственньм прообразом дивизора $A+H$. Если $H$ обилен, то $a H+A$ обилен для $a \gg 0$, и поскольку $H_{r}(\widetilde{X})=H_{r}(X) \oplus H_{r-2}(S)$, следствие 2.2.2 принимает следуюший вид:

2.2.3. ПРЕДЛОЖЕНИЕ. Пусть $A \subset X-$ гладкий дивизор, $H \subset X-$ обильный дивизор, трансверсальный $\kappa A$, и $\widetilde{Y}_{H}$ - указанное выше многообразие. Тогда 
для любого поля $F$ c char $F \neq 2$ и для любого $r \in \mathbb{Z}$ имеются канонические изоморфизмы $H_{r}^{+}\left(\widetilde{Y}_{H} ; F\right)=H_{r}(X ; F) \oplus H_{r-2}(H \cap A ; F)$ u $H_{+}^{r}\left(\widetilde{Y}_{H} ; F\right)=H^{r}(X ; F) \oplus$ $H^{r-2}(H \cap A ; F)$. Более того, $H_{r}^{-}\left(\widetilde{Y}_{H} ; F\right)=H_{-}^{r}\left(\widetilde{Y}_{H} ; F\right)=0$ для $r \neq n=\operatorname{dim} X$. (Здесь $H_{*}^{ \pm}$и $H_{ \pm}^{*}$ - подгруппь классов, инвариантных и косоинвариантных относительно автоморфизма накрытия.)

2.2.4. Пусть теперь $X$ наделено вешественной структурой и $A$ - четный вещественньй дивизор. Пусть, далее, дивизор $E$ с $2 E \sim A$ выбран также вещественным. Положим $Y=Y(E)$. (Такой выбор дивизора $E$ возможен, если, например, $H^{1}(X)=0$; в обшем случае возможность выбора зависит от того, является ли класс дивизора $A$ в $H^{2}(X ; \mathbb{Z})$ удвоенным косоинвариантным классом.) Если $\mathbb{R} X \neq \varnothing$, инволюция сопј на $X$ накрывается двумя антиголоморфными инволюциями $T_{ \pm}: Y \rightarrow Y$, коммутирующими друг с другом, причем $\tau=T_{+} \circ T_{-}$, где $\tau: Y \rightarrow Y$ - автоморфизм накрытия (см. А.2.5). Соответствуюшие вешественные части многообразия $Y$ обозначаются через $\mathbb{R} Y_{ \pm}$, а их проекции на $\mathbb{R} X$ - через $\mathbb{R} X_{ \pm}$. Очевидно, $\mathbb{R} X_{+} \cup \mathbb{R} X_{-}=\mathbb{R} X$ и $\mathbb{R} X_{+} \cap \mathbb{R} X_{-}=\mathbb{R} A$. Характеристические классы $\omega_{ \pm} \in H^{1}\left(\mathbb{R} X_{ \pm}\right)$двойственны по Пуанкаре соответствуюшим образам $\operatorname{rel}[\mathbb{R} E] \in H_{n-1}\left(\mathbb{R} X_{ \pm}, \mathbb{R} A\right)$ при релятивизации.

2.3. Неравенства Смита. Результаты этого пункта, полученные из неравенства Смита и его относительного варианта (см. А.1.3(2) и (А.1.9)), обобщают неравенство Харнака для кривых.

2.3.1. ТеоремА. Если $X$ - неособое многообразие с вещественной структурой, то $\beta_{*}(\mathbb{R} X) \leqslant \beta_{*}(X)$ u $\beta_{*}(\mathbb{R} X)=\beta_{*}(X) \bmod 2 . \quad$ Если, кроме того, $A \subset X$ - вещественное неособое подмногообразие, то $\beta_{*}(\mathbb{R} X, \mathbb{R} A) \leqslant \beta_{*}(X, A)$ u $\beta_{*}(\mathbb{R} X, \mathbb{R} A)=\beta_{*}(X, A) \bmod 2$.

В 2.3.1 легко угадываются привычные соотношения между числом вешественных корней вешественного многочлена и его степенью, если $\operatorname{dim} X=0$, и классическое неравенство Харнака, которое утверждает, что число вешественных компонент кривой $\mathbb{R} X$ не превосходит род $(X)+1$, если $\operatorname{dim} X=1$. Оценка, которую дает 2.3.1, точна для гиперповерхностей любой степени в проективном пространстве любой размерности (см. [118] и [53]); для гиперповерхности $X \subset \mathbb{P}^{q}$ степени $m$ эта оценка имеет вид $\beta_{*}(X) \leqslant \frac{1}{m}\left((m-1)^{q+1}+(-1)^{q}\right)+q-(-1)^{q}$, см. [113].

2.3.2. ДОПОЛНИТЕЛЬНОЕ НЕРАВЕНСТВО ДЛЯ ЧЕТНЫХ ПАР. Пусть $A-$ четный обильный вещественный гладкий дивизор на неособом $n$-мерном многообразии $X$ с вещественной структурой. Пусть, далее, $E$-вещественный дивизор на $X с$ $2 E \sim A u \omega_{ \pm} \in H^{1}\left(\mathbb{R} X_{ \pm}\right)$- класс, двойственный по Пуанкаре классу $\operatorname{rel}[\mathbb{R} E] \in$ $H_{n-1}\left(\mathbb{R} X_{ \pm}, \mathbb{R} A\right)$, где $\mathbb{R} X_{ \pm}$- две половинь пространства $\mathbb{R} X \backslash \mathbb{R} A$ (см. 2.2.4). Тогда

$$
2 \beta_{*}\left(\mathbb{R} X_{ \pm}\right)-2\left[\operatorname{dim} \operatorname{Ker} \partial-\operatorname{dim} \operatorname{Ker}\left(\omega_{ \pm} \oplus \partial\right)\right] \leqslant \beta_{*}(X)+(-1)^{n}[\chi(X)-\chi(A)]
$$

где $\omega_{ \pm} \oplus \partial: H_{*}\left(\mathbb{R} X_{ \pm}, \mathbb{R} A\right) \rightarrow H_{*-1}\left(\mathbb{R} X_{ \pm}, \mathbb{R} A\right) \oplus H_{*-1}(\mathbb{R} A)$ - граничныи гомоморфизм точной последовательности Смита А.1.1.

ДоказАТЕльство. Это утверждение есть неравенство Смита, примененное к одной из инволюций $T_{ \pm}$двулистного разветвленного накрывающего $Y \rightarrow X$ с ветвлением над $A$ (см. 2.2.4). Его левая часть есть $\beta_{*}\left(\mathbb{R} Y_{ \pm}\right)$, как это сразу следует из точной 
последовательности Смита для автоморфизма накрытия, точной последовательности пары $(\mathbb{R} X, \mathbb{R} A)$ и двойственности Пуанкаре. Его правая часть равна $\beta_{*}(Y)$. Действительно, из 2.2.2 и из симметричности чисел Бетти следует, что $\beta_{r}(Y)=\beta_{r}(X)$ для всех $r \neq n$, и для нахождения оставшегося числа Бетти $\beta_{n}(Y)$ достаточно сравнить соответствующие эйлеровы характеристики (используя, например, формулу Римана-Гурвища А.1.3(6)).

ЗАмечание. Левая часть неравенства в 2.3.2 равна $\beta_{*}(\mathbb{R} A)+2 \operatorname{dim} \operatorname{Ker}\left(\omega_{ \pm} \oplus \partial\right)$. В случае проективной гиперповерхности это неравенство можно упростить, см. 2.8.3; в этом случае $\omega_{ \pm}$есть $k$-кратная образующая группы $H^{1}\left(\mathbb{R P}^{n}\right)$, где $2 k=\operatorname{deg} A$. (По странной ошибке в ранних работах на эту тему был указан другой класс.)

В отличие от случая кривых, в общем случае оценка отдельного числа $\beta_{i}(\mathbb{R} X)$ (и даже $\left.\beta_{0}(\mathbb{R} X)\right)$ - трудная проблема; точная граница неизвестна даже для поверхностей степени 5 в $\mathbb{P}^{3}$, см. 3.5. Заметим также, что 2.3.1 и 2.3.2 дают, вообще говоря, разные ограничения на топологию пары $(\mathbb{R} X, \mathbb{R} A)$. Конечно, условие обильности в 2.3.2 необходимо только для нахождения чисел Бетти многообразия $Y$; без него правую часть неравенства следует заменить на $\beta_{*}(Y)$.

Согласно 2.3.1 разность $\beta_{*}(X)-\beta_{*}(\mathbb{R} X)$ есть неотрицательное четное число. Если эта разность равна $2 d$, то $X$ назьвается $(M-d)$-многообразием (а conj назьвается $(M-d)$-инволюиией). Подобно случаю кривых и классическому неравенству Харнака, именно $M$ - (и близкие к $M$-) многообразия удовлетворяют соответствуюшим запретам типа сравнений (см. 2.7).

2.4. Неравенства Комессатти-Петровского-Олейник. Напомним, что если на неособом компактном комплексном многообразии $X$ существует кэлерова метрика (что справедливо для проективных неособых многообразий, так как кэлерова метрика на них индуцируется из объемлющего проективного пространства), то определено каноническое разложение Ходжа (структура Ходжа)

$$
H^{r}(X, \mathbb{C})=\bigoplus_{p+q=r} H^{p, q}(X), \quad 0 \leqslant p, q \leqslant \operatorname{dim}_{\mathbb{C}} X
$$

где $H^{p, q}(X)=H^{q}\left(X ; \Omega^{p}(X)\right)$ есть одновременно пространство классов, реализуемых $(p, q)$-формами, и пространство $q$-когомологий пучка $\Omega^{p}(X)=\bigwedge^{p} T^{*}(X)$ голоморфных $p$-форм на $X$. Числа Ходжса $h^{p, q}(X)=\operatorname{dim} H^{p, q}(X)$ инвариантны относительно деформаций многообразия $X$. Фиксированная кэлерова метрика дает дальнейшее разложение $H^{p, q}(X)=\bigoplus \Omega^{k} \wedge P^{p-k, q-k}(X)$, где $\Omega \in H^{1,1}(X)$ - фундаментальный класс метрики и $P^{a, b} \subset H^{a, b}$ - подпространства так называемых примитивных классов (см., например, [16]). Если многообразие $X$ вещественное, на нем существует инвариантная кэлерова метрика (например, полученная усреднением) и для такой метрики справедливо равенство соnj* $\Omega=-\Omega$.

Следуюший результат содержит неравенства Петровского [94], Петровского-Олейник [95], [91] и Комессатти [17] (будучи примененным соответственно к двулистньм накрытиям плоскости, проективным гиперповерхностям двулистным накрытиям поверхностей и поверхностям). Другие обобщения неравенств Петровского-Олейник можно найти в [3], [65], [66], [58], [116].

Приведенное ниже доказательство взято из [56]. 
2.4.1. Теорема. Если $X$ - неособое компактное комплексное кәлерово многообразие четной размерности $n=2 k$, наделенное вещественной структурой, mo

$$
|\chi(\mathbb{R} X)-1| \leqslant h^{k, k}(X)-1
$$

Eсли, кроме того, $A \subset X-$ такой четный вещественный дивизор, что $A \sim 2 E$ для вещественного дивизора $E$, то

$$
\begin{gathered}
\left|\chi\left(\mathbb{R} X_{-}\right)-\chi\left(\mathbb{R} X_{+}\right)\right| \leqslant h_{-}^{k, k}(Y) \quad u \\
\left|2 \chi\left(\mathbb{R} X_{ \pm}\right)-1\right| \leqslant h^{k, k}(Y)-1,
\end{gathered}
$$

где $Y=Y(E), \tau$ - автоморфизм накрытия $и h_{-}^{p q}(Y)=h^{p, q}(Y)-h^{p, q}(X)$ - размерность косоинвариантной относительно $\tau$ части $H_{-}^{p q}(Y) \subset H^{p, q}(Y)$.

ДокАЗАтЕльство. Докажем первое утверждение, два других доказываются аналогично. По теореме Лефшеца о неподвижных точках

$$
\chi(\mathbb{R} X)=\sum_{r}(-1)^{r} \operatorname{Trace}\left(\operatorname{conj}^{*}, H^{r}(X)\right)
$$

Так как $\operatorname{conj}_{*} H^{p, q}(X)=H^{q, p}(X)$ и соnj* $\Omega=-\Omega$ (для инвариантной кэлеровой метрики), то при разложении пространства $H^{*}(X ; \mathbb{C})$ на примитивные классы вклад в $\chi(\mathbb{R} X)$ могут дать только члены, содержащиеся в $H^{k, k}(X)=\bigoplus \Omega^{j} \wedge P^{k-j, k-j}(X)$ :

$$
\chi(\mathbb{R} X)=\sum_{j=0}^{k} \operatorname{Trace}\left(\operatorname{conj}^{*}, P^{k-j, k-j}(X)\right) .
$$

Остается заметить, что conj* $=$ id на $P^{0,0}(X)$ и что $\mid$ Trace $\mid \leqslant \operatorname{dim}$.

Неравенства, указанные в этой теореме, точны для кривых на $\mathbb{P}^{2}$ (см. [94]) и для поверхностей в $\mathbb{P}^{3}$ (см. [118]); в этих случаях они являются соответственно неравенствами Петровского и Петровского-Олейник. Насколько нам известно, в старших размерностях вопрос их точности до сих пор открыт.

Числа Ходжа. Если $A$ обилен, последние два неравенства в 2.4 .1 можно сделать эффективньми. Следуя $\Phi$. Хиребруху, рассмотрим обобщенный род Тодда (или просто $T_{y}$-род) $T_{y}(X)=\sum(-1)^{q} h^{p, q}(X) y^{p}$. По теореме Римана-Роха-Хирцебруха (см. [49]) $T_{y}(X)$ равен значению на $[X]$ некоторого многочлена $T_{n}\left(y ; c_{1}, \ldots, c_{n}\right)$ степени $n$ от классов Черна $c_{i}=c_{i}(X)$. Более того, для любых $u_{1}, \ldots, u_{r} \in H^{2}(X)$ можно определить виртуальный род Тодда $T_{y}^{r}\left(u_{1}, \ldots, u_{r}\right)$, являюшийся многочленом от $c_{i}$ и $u_{j}$ со следующим свойством: если классы, двойственные по Пуанкаре классам $u_{i}$, реализованы неособыми подмногообразиями $U_{i}$ коразмерности 1 , пересекающимися трансверсально, то $T_{y}^{r}\left(u_{1}, \ldots, u_{r}\right)=T_{y}\left(U_{1} \cap \cdots \cap U_{r}\right)$.

Следуюшее утверждение следует из 2.2.2 (с $G=\mathbb{C})$ : 
2.5.1. ПРЕДЛОЖЕНИЕ (см. [56]). Пусть $A \subset X u Y=Y(E)-$ maкuе же, как $u$ в 2.4.1. Если А обилен, то $h^{p, q}(Y)=h^{p, q}(X)$ для всех $p, q$ с $p+q \neq n u$

$$
\sum_{i=0}^{n}(-1)^{i}\left(h^{n-i, i}(Y)-h^{n-i, i}(X)\right) y^{i}=\sum_{r \geqslant 0} a_{r}(y) T_{y}^{r}(e, \ldots, e)_{X}-T_{y}(X),
$$

где $a_{r}(y)$ - коэффициент при $x^{r}$ в формальном степенном ряде

$$
\frac{(1+y x)^{2}-(1-x)^{2}}{(1+y x)^{2}+y(1-x)^{2}} \cdot \frac{1}{x}=\sum_{r=0}^{\infty} a_{r}(y) x^{r}
$$

и е $=c_{1}(E)$. (Этот результат не зависит от выбора дивизора $E$ с $2 E \sim A$, так как вычисления происходят в рациональных гомологиях.)

ДокАЗАТЕЛЬСТво. Из 2.2.2, естественности разложения Ходжа и двойственности Серра следует, что $h^{p, q}(Y)=h^{p, q}(X)$ для $p+q \neq n$, и для нахождения оставшихся чисел Ходжа в средней размерности достаточно знать род Тодда многообразия $Y$, который, согласно [56], есть $T_{y}(Y)=\sum_{r \geqslant 0} a_{r}(y) T_{y}^{r}(e, \ldots, e)_{X}$.

Непосредственные вычисления для малых размерностей дают следующие значения $T_{y}(X)=\sum_{i=0}^{n} T_{n, i} y^{i}$ (где $\left.n=\operatorname{dim} X\right)$ :

2.5.3. СлЕДСТвИЕ. Пусть $c_{i}=c_{i}(X)$. Если $\operatorname{dim} X=2, m o$

$$
\begin{gathered}
T_{2,0}=T_{2,2}=\frac{1}{12}\left(c_{1}^{2}+c_{2}\right)[X], \quad T_{2,1}=\frac{1}{6}\left(c_{1}^{2}-5 c_{2}\right)[X], \\
h^{1,1}(Y)=h^{1,1}(X)-T_{2,1}-\left(e c_{1}-3 e^{2}\right)[X] .
\end{gathered}
$$

Ecлu $\operatorname{dim} X=4, m o$

$$
\begin{gathered}
T_{4,0}=T_{4,4}=\frac{1}{720}\left(-c_{1}^{4}+4 c_{1}^{2} c_{2}+3 c_{2}^{2}+c_{1} c_{3}-c_{4}\right)[X], \\
T_{4,1}=T_{4,3}=\frac{1}{180}\left(-c_{1}^{4}+4 c_{1}^{2} c_{2}+3 c_{2}^{2}-14 c_{1} c_{3}-31 c_{4}\right)[X], \\
T_{4,2}=\frac{1}{120}\left(-c_{1}^{4}+4 c_{1}^{2} c_{2}+3 c_{2}^{2}-19 c_{1} c_{3}+79 c_{4}\right)[X], \\
h^{2,2}(Y)=h^{2,2}(X)+T_{4,2}+\frac{1}{12}\left(115 e^{4}-46 e^{3} c_{1}-5 e^{2} c_{1}^{2}+31 e^{2} c_{2}+e c_{1} c_{2}-12 e c_{3}\right)[X] .
\end{gathered}
$$

Если $X$ - регулярное полное пересечение в $\mathbb{P}^{N}$, его числа Ходжа и, следовательно, правые части неравенств в 2.4.1 можно найти по рекуррентной формуле. Они зависят только от полистепени многообразия $X$. Определим многочлены $\chi_{s}^{q}\left(m_{1}, \ldots, m_{s} ; y\right)$ следующим образом:

$$
\begin{aligned}
\chi_{0}^{0}(y) & =1, \quad \chi_{s}^{0}\left(m_{1}, \ldots, m_{s} ; y\right)=0 \text { для } s>0 \text { и } \\
\chi_{s}^{q}\left(\ldots, m_{s} ; y\right)= & m_{s} \chi_{s-1}^{q-1}(\ldots ; y) \\
& +\sum_{\mu=1}^{m_{s}}\left[(y-1) \chi_{s}^{q-1}(\ldots, \mu-1 ; y)-y \chi_{s+1}^{q-1}(\ldots, \mu-1, \mu ; y)\right] .
\end{aligned}
$$

(Заметим, что $\chi_{0}^{q}(y)=\sum_{r=0}^{q}(-1)^{r} y^{r}, \quad \chi_{q}^{q}\left(m_{1}, \ldots, m_{q} ; y\right)=m_{1} \cdots m_{q}$ и $\chi_{s}^{q}=0$ для $s>q$.) 
2.5.5. ПРЕДЛОЖЕНИЕ. Если неособое многообразие $X$ четной размерности $n=2 l$ есть регулярное полное пересечение в $\mathbb{P}^{n+s}$ полистепени $\left(m_{1}, \ldots, m_{s}\right)$, то

$$
T_{y}(X)=\chi_{s}^{n+s}\left(m_{1}, \ldots, m_{s} ; y\right)
$$

В частности, $(-1)^{l} h^{l, l}(X)$ равно коэффичиенту при $y^{l}$ в $\chi_{s}^{n+s}\left(m_{1}, \ldots, m_{s} ; y\right)$.

Далее, если $A \subset X$-неособое подмногообразие, высекаемое на $X$ гиперповерхностью степени $2 k$, и $Y$-двулистное накрывающее многообразия $X$ с ветвлением над $A$, то

$$
T_{y}(Y)=\sum_{r=0}^{n} a_{r}(y) \chi_{r+s}^{n+s}\left(m_{1}, \ldots, m_{s}, k, \ldots, k ; y\right)
$$

(Числа $a_{r}(y)$ определены в (2.5.2).) В частности, $(-1)^{l} h^{l, l}(Y)$ равно коэффициенту при $y^{l}$ в әтом многочлене.

ДокАЗАТЕльство (см. [56]). Это утверждение следует непосредственно из функционального уравнения для $T_{y}$-рода [ 49 ; теорема 11.3 .1$]$ и хорошо известного свойства чисел Ходжа: $h^{p, q}(X)=0$, если $p \neq q$ и $p+q \neq n$.

В малых размерностях (поверхности в $\mathbb{P}^{q}$ и гиперповерхности в $\mathbb{P}^{5}$ ) предложение 2.5.5 дает следующий результат (для упрощения формул $i$-й элементарный симметрический многочлен от $m_{1}, \ldots, m_{s}$ мы обозначаем через $\mu_{i}$; ясно, что $\mu_{0}=1$ и $\mu_{i}=0$ для $\left.i>s\right)$.

2.5.6. СЛЕДСТВИЕ. Если $X \subset \mathbb{P}^{q} u \operatorname{dim} X=2, m o$

$$
\begin{gathered}
h^{1,1}(X)=\frac{1}{12} \mu_{q-2}\left(8 \mu_{1}^{2}-10 \mu_{2}-6(q+1) \mu_{1}+(q+1)(3 q-2)\right), \\
h^{1,1}(Y)=\mu_{q-2} k\left(3 k+\mu_{1}-(q+1)\right)+2 h^{1,1}(X) .
\end{gathered}
$$

Ecлu $X \subset \mathbb{P}^{5} u \operatorname{dim} X=4, m o$

$$
\begin{aligned}
& s=0: \quad h^{2,2}(Y)=\frac{115}{12} k^{4}-\frac{115}{6} k^{3}+\frac{185}{12} k^{2}-\frac{35}{6} k+2, \\
& s=1: \quad h^{2,2}(X)=\frac{11}{20} m_{1}^{5}-\frac{11}{4} m_{1}^{4}+\frac{23}{4} m_{1}^{3}-\frac{25}{4} m_{1}^{2}+\frac{37}{10} m_{1} \text {, } \\
& h^{2,2}(Y)=\frac{115}{12} m_{1} k^{4}+\left(\frac{23}{6} m_{1}-23\right) m_{1} k^{3} \\
& +\left(\frac{13}{6} m_{1}^{2}-\frac{21}{2} m_{1}+\frac{95}{4}\right) m_{1} k^{2} \\
& +\left(\frac{11}{12} m_{1}^{3}-5 m_{1}^{2}+\frac{43}{4} m_{1}-\frac{25}{2}\right) m_{1} k+2 h^{2,2}(X) \text {. }
\end{aligned}
$$

ЗАмечАниЕ. В формулировках Петровского-Олейник вместо чисел Ходжа использовался подсчет числа некоторых мономов. Совпадение двух оценок было доказано В.И. Звониловьм [128] с использованием 2.5.1. Этот результат связан со следующим красивым правилом: если $X \subset \mathbb{P}^{2 k+1}$ - гиперповерхность с $\operatorname{deg} X=d$, то $h^{k, k}(X)-1$ есть число целых точек в слое $k d<\sum\left(x_{i}+1\right)<(k+1) d$ куба $[0, d-2]^{2 k+1} \subset \mathbb{R}^{2 k+1}$. Другие слои куба дают другие числа Ходжа. Эта формула распространяется на гиперповерхности в пространствах четной размерности. Соотношения такого типа были впервые замечены, по-видимому, Стинбринком [108], 
который нашел в явном виде мономиальный базис для исчезающей структуры Ходжа полуквазиоднородной особенности. Дальнейшие обобшения см. в [22] и [115].

2.6. Типы инволюций. В теории вешественных кривых понятие разбивающей кривой (или кривой типа I, см. введение) является одним из основных. В старших размерностях, где вешественная часть $\mathbb{R} X$ имеет (вещественную) коразмерность больше 1 , она не может разбивать многообразие $X$, но гомологическое условие $[\mathbb{R} X]=0 \in$ $H_{n}(X)$ по-прежнему имеет смысл. В различных приложениях свойства пространства $\mathbb{R} X$ могут зависеть от того, гомологичен ли его фундаментальньй класс некоторому классу $u \in H_{n}(X)$, обычно обладаюшему некоторым свойством естественности (скажем, характеристическому классу многообразия $X$ ) и не обязательно нулевому. Таким образом, для $u \in H_{n}(X)$ будем говорить, что вещественная структура conj на $X$ (или просто само $X$, или $\mathbb{R} X$ ) имеет $m u n \mathrm{I}_{u}$, если $[\mathbb{R} X]$ гомологичен классу $u$. Следующие специальные случаи используются наиболее часто.

(1) $\mathrm{I}_{0}$ или $\mathrm{I}_{\mathrm{abs}} \mathrm{c} u=0$. Тип $\mathrm{I}_{0}$ иногда называют абсолютнымм типом $\mathrm{I}$.

(2) $\mathrm{I}_{\text {wu}}$, где $u=u_{n}(X)$ есть $n$-й класс Ву многообразия $X$. (Напомним, что $u_{n}(X)$ есть характеристический элемент формы пересечений на $X$. По формуле Ву $u_{n}(X)$ есть некоторый многочлен от классов Штифеля-Уитни многообразия $\left.X^{2}\right)$.

(3) $\mathrm{I}_{\mathrm{hp}}$ c $u$, равным $(n / 2)$-й степени гиперплоского сечения. (Конечно, это имеет смысл только для фиксированного вложения $X \hookrightarrow \mathbb{P}^{N}$.)

В случае проективных многообразий понятие типов $\mathrm{I}_{0}$ и $\mathrm{I}_{\mathrm{hp}}$ появилось в [59]. В [117] типы $\mathrm{I}_{0}$ и $\mathrm{I}_{\mathrm{hp}}$ называются соответственно $\mathrm{I}_{\mathrm{abs}}$ и $\mathrm{I}_{\mathrm{rel}}$, а гиперповерхность, не относяшаяся ни к одному из этих типов, относится к типу II. Однако эта терминология не представляется обшепризнанной. Обычно в зависимости от конкретной проблемы является разумным выделить класс $u \in H_{n}(X)$ и относить многообразия к типам $\mathrm{I}_{\mathrm{abs}}$ и $\mathrm{I}_{u}=\mathrm{I}_{\mathrm{rel}}$, заменяя последним тип II. Например, ниже в большинстве результатов существенную роль играет тип $\mathrm{I}_{\mathrm{wu}}$.

2.7. Сравнения. Следуюшие две теоремы являются прямьми обобщениями сравнений Гудкова, Арнольда, Рохлина, Харламова и Крахнова.

2.7.1. ЭКСТРЕМАЛЬНЫЕ СРАВНЕНИЯ. Пусть $X-$ четномерное $(M-d)$-многообразие с вещественной структурой. Тогда:

(1) если $d=0, \operatorname{mo} \chi(\mathbb{R} X)=\sigma(X) \bmod 16$;

(2) если $d=1, \operatorname{mo} \chi(\mathbb{R} X)=\sigma(X) \pm 2 \bmod 16$;

(3) если $d=2$ и $\chi(\mathbb{R} X)=\sigma(X)+8 \bmod 16$, mо $X$ имеет mun $\mathrm{I}_{\mathrm{wu}}$.

2.7.2. ОБОБШЕННОЕ СРАВНЕНИЕ АРНОЛЬДА. Пусть $X$ - неособое многоббрзие с вещественной структурой типа $\mathrm{I}_{\mathrm{wu}}$. Если размерность многообразия $X$ четна, то $\chi(\mathbb{R} X)=\sigma(X) \bmod 8$.

2.7.3. ПреДЛОЖЕнИЕ. $М$-многообразие $с$ вещественной структурой имеет mun $\mathrm{I}_{\mathrm{wu}}$.

Сигнатура комплексно-аналитическогомногообразия $X$ равна $T_{1}(X)$ (см. 2.4). Поэтому если $X$ - поверхность, то $\sigma(X)=\frac{1}{3}\left(c_{1}^{2}-2 c_{2}\right)[X]$. Если $X-$ регулярное полное

\footnotetext{
${ }^{2}$ Так как $X$ - комплексное многообразие, то $w_{2 i+1}(X)=0$ и $w_{2 i}(X)=c_{i}(X) \bmod 2$. Заметим, что $\operatorname{conj}_{*} c_{i}=(-1)^{i} c_{i}$; в частности, все характеристические классы $\bmod 2$ многообразия $X$ являются сопj ${ }_{*}$-инвариантными.
} 
пересечение в $\mathbb{P}^{q}$, его сигнатуру можно найти, используя предложение 2.5.5 и формулу (2.5.4). В частности, получаются следуюшие результаты:

2.7.4. СИГНАТУРА ПОЛНОГО ПЕРЕСЕЧЕНИЯ. Пусть $X$ - регулярное полное пересечение в $\mathbb{P}^{q}$ полистепени $\left(m_{1}, \ldots, m_{s}\right)$ и четной размерности $n=q-s$.

(1) $\operatorname{Ecли~} n=2$, то $\sigma(X)=-\frac{1}{3} \mu_{q-2}\left(\mu_{1}^{2}-2 \mu_{2}-q-1\right)$, где $\mu_{i}-i$-й әлементарный симметрический многочлен от $m_{1}, \ldots, m_{s}$.

(2) Eсли $s=q+2 \bmod 4 u m_{1}+\cdots+m_{s}=q+1 \bmod 2, \operatorname{mo} \sigma(X)=0 \bmod 16$.

(3) Eсли $s=q \bmod 4$ и все $m_{i}$, кроме, может быть, одного $\left(\right.$ скажем, $\left.m_{s}\right)$, нечетнй, то $\sigma(X)=m_{1} \cdots m_{s} \bmod 16$.

(4) Если $X$ - гиперповерхность (т.е. $s=1)$, то $\sigma(X)=m_{1}(\bmod 16)$ npu $n=0 \bmod 4$; $\sigma(X)=0 \bmod 16$ при $n=2 \bmod 4$ и $m_{1}$ четном; $\sigma(X)=1-m_{1}\left(m_{1}-1\right) \bmod 16$ при $n=2 \bmod 4$ и $m_{1}$ нечетном.

ДокАЗАТЕльство. Воспользуемся равенством $\sigma(X)=\chi_{s}^{q}\left(m_{1}, \ldots, m_{s} ; 1\right)$. $\mathrm{У}_{\mathrm{T}-}$ верждение (1) доказьвается по индукции с использованием (2.5.4). Утверждение (2) следует из того, что сигнатура $(8 k+4)$-мерного спинорного многообразия делится на 16 (см. [90]). Сравнение (3) доказьвается индукцией по $s$ и $m_{s}$ с использованием функционального уравнения для $T_{y}$-рода [49; теорема 11.3.1)], согласно которому

$(2.7 .5) \chi_{s}^{q}\left(\ldots, m_{s} ; 1\right)=\chi_{s}^{q}\left(\ldots, m_{s}-1 ; 1\right)+\chi_{s-1}^{q-1}(\ldots ; 1)-\chi_{s+1}^{q-1}\left(\ldots, m_{s}-1, m_{s} ; 1\right)$.

Из условий (3) в силу (2) последнее слагаемое в (2.7.5) сравнимо с нулем.

Утверждения (2) и (3) охватывают первые два случая в (4). В последнем случае мы опять используем $(2.7 .5)$, где $\chi_{1}^{q}(m-1 ; 1)=0 \bmod 16$ согласно $(2), \chi_{0}^{q-1}(1)=$ $\sigma\left(\mathbb{P}^{q-1}\right)=1$ и $\chi_{2}^{q-1}(m-1, m ; 1)=(m-1) m \bmod 16$ согласно $(3)$.

Доказательства утверждений 2.7.1-2.7.3 похожи друг на друга; ключевой вклад дает лемма 2.7.6 (см. ниже).

Пусть $n=\operatorname{dim} X$. Положим $H=H_{n}(X ; \mathbb{Z}) /$ Tors. Пусть $H^{ \pm 1} \subset H-$ подгруппы

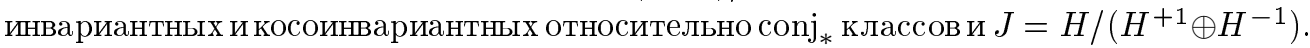
Обозначим через $B: H \otimes H \rightarrow \mathbb{Z}$ форму пересечений $(x, y) \mapsto x \circ y$ многообразия $X$ и через $B^{\text {conj }}$ - скрученную форму пересечений $(x, y) \mapsto x \circ \operatorname{conj}_{*} y$, см. А.3. Пусть $q^{ \pm}$- дискриминантная группа (квадратичное пространство) формы, ассоциированной с $\left.B\right|_{H^{ \pm}}$. Как известно (см. В.3), $J$ и $q^{ \pm}-\mathbb{Z}_{2}$-векторные пространства, изоморфные как группы.

2.7.6. Лемма. Пусть, как и выше, $X$ есть $(M-d)$-многообразие с вещественной структурой $u \operatorname{dim} X=n$. Тогда:

(1) $[\mathbb{R} X]$ как әлемент в $H_{n}(X)$ является характеристическим для скрученной формы пересечений $B^{\mathrm{conj}}$;

(2) $u_{n}(X) u[\mathbb{R} X] \in H_{n}(X)$ - челочисленные классьи, т.е. принадлежсат образу әруппьь $H_{n}(X ; \mathbb{Z})$;

(3) $d \geqslant \operatorname{dim}$ Coker $\left[\left(1+\operatorname{conj}_{*}\right): H_{*}(X) \rightarrow H_{*}(X)\right] \geqslant \operatorname{dim} J$.

Пусть, кроме того, размерность $n=2 k$ четна. Положим $\epsilon=(-1)^{k+1}$. Тогда

(4) $\chi(\mathbb{R} X)=(-1)^{k+1} \sigma\left(B^{\text {conj }}\right)=\sigma(X)-2 \sigma\left(H^{\epsilon}\right)$;

(5) $H^{\epsilon}$ - четная решетка;

(6) $\operatorname{dim} J=d \bmod 2$. 
ДокАЗАтЕльство. Утверждение (1) есть лемма Арнольда А.3.3; (4) сразу следует из А.3.2; $(5)$ следует из того, что $u_{n}(X)$, как и любой характеристический класс многообразия $X$, реализуется формальной линейной комбинацией вешественных циклов размерности $k$ (см., например, [101]) и, следовательно, принадлежит подгруппе $H^{-\epsilon}$.

Чтобы доказать (2), заметим, что как стандартная, так и скрученная $\mathbb{Z}_{2}$-формы пересечений равны нулю на образе кручения $\operatorname{Tors} H_{n}(X ; \mathbb{Z})$ (что справедливо и для соответствуюших $\mathbb{Z}$-форм). Следовательно, их характеристические классы аннулируют Tors $H_{n}(X ; \mathbb{Z})$ и потому целочисленны.

Утверждение (3) следует из А.1.3(1) и из геометрической конструкции точной последовательности Смита (см. А.1.5). Действительно, Ker pr ${ }_{*}$ в А.1.1 состоит из геометрически инвариантных и, следовательно, conj $_{*}$-инвариантных циклов, которые, таким образом, принадлежат $\operatorname{Ker}\left(1+\operatorname{conj}_{*}\right)$. Это доказьвает первое неравенство в (3); второе неравенство очевидно, так как $\operatorname{dim} J$ равна $\operatorname{dim} \operatorname{Ker}\left(1+\operatorname{conj}_{*}\right)$ для инволюции, рассматриваемой на $H \otimes \mathbb{Z}_{2}$.

Доказательство утверждения (6) основано на следующих двух леммах:

2.7.7. ЛЕмма. Если замкнутое многообразие $M$ имеет четную вещественную размерность $n=2 k$, то $\beta_{*}(M)=(-1)^{k} \chi(M) \bmod 4$.

ДокАЗАТЕЛЬСТво. Если $k=2 l$ четно, то в силу двойственности Пуанкаре

$$
\beta_{*}(M)-\chi(M)=2 \sum_{r=0}^{k-1} \beta_{2 r+1}(M)=4 \sum_{r=0}^{l-1} \beta_{2 r+1}(M) .
$$

Доказательство для нечетного $k$ аналогично.

2.7.8. ЛЕМмА. Если $X$-неособое замкнутое комплексное многообразие четной комплексной размерности $n=2 k$, mо $\sigma(X)=(-1)^{k} \chi(X) \bmod 4$.

ДокАЗАТЕльство. Эта лемма доказывается аналогично предыдущей с использованием $T_{y}$-характеристики (см. 2.4), равенств $\sigma(X)=T_{1}(X)$ и $\chi(X)=T_{-1}(X)$ и симметрии $T_{n, i}=(-1)^{n} T_{n, n-i}$ коэффишиентов $T_{n, i}$ в $T_{y}(X)=\sum T_{n, i} y^{i}$ (см. [49; п. 1.8]; заметим, что $T_{n, i}$ принимают целые значения на любом, не обязательно кэлеровом, комплексном многообразии).

Леммы 2.7.7 и 2.7.8, примененные к многообразию $X$, дают сравнение $\beta_{*}(X)=$ $(-1)^{k} \sigma(X) \bmod 4$. Поэтому $(4)$ преврашается в $(-1)^{k} \beta_{*}(\mathbb{R} X)=(-1)^{k} \beta_{*}(X)-2 \sigma\left(H^{\epsilon}\right)$ $\bmod 4$ и (6) следует из сравнения $\sigma\left(H^{\epsilon}\right)=\operatorname{dim} q^{\epsilon} \bmod 2$ (эта решетка четна согласно (5)) и равенства $\operatorname{dim} J=\operatorname{dim} q^{ \pm}$.

ЗАмечАнИЕ. Лемму 2.7.8 можно распространить на почти комплексные многообразия, воспользовавшись формулой Атийи-Дюпона [8] (см. [126]) или кобордизмами.

ДОКАЗАТЕЛЬСТВо ТЕОРЕмы 2.7.1. Из (3) и (6) в 2.7.6 следует, что $\operatorname{dim} q^{\epsilon}=\operatorname{dim} J$ $=0$ или 1 (где по-прежнему $\epsilon=(-1)^{k+1}$ ), если вьполнены условия соответственно (1) или (2) в 2.7.1. Поэтому 2.7.1(1) и (2) следуют из В.2.2, примененного к $H^{\epsilon}$ в 2.7.6(4).

Аналогичные соображения показывают, что в условиях 2.7.1(3) дискриминантные формы $q^{ \pm}$, размерности которых не превосходят 2, четны и согласно В.3.1 разность $[\mathbb{R} X]-u_{n}(X)$ аннулирует все целочисленные классы в $H_{n}(X)$, т.е. принадлежит 
образу кручения Tors $H_{n}(X ; \mathbb{Z})$. Поэтому, опять используя 2.7.6(3), где оба неравенства обрашаются в равенства, заключаем, что каждый элемент группы $H_{n}(X) / H_{n}(X ; \mathbb{Z}) \otimes \mathbb{Z}_{2}$ имеет conj ${ }_{*}$-инвариантного представителя. Следовательно, $B$ и $B^{\text {conj }}$ совпадают на таких классах и разность $u_{n}(X)-[\mathbb{R} X]$ равна нулю.

ДокАЗАТЕЛЬСТВо ТЕОРЕМЫ 2.7.2. Так как $[\mathbb{R} X]$ и $u_{n}(X)$ совпадают, то из В.3.1 следует, что $q^{ \pm}$- четные дискриминантные решетки. Следовательно, $\mathrm{Br} q^{ \pm}=0 \bmod 4$ и применимо утверждение 2.7.6(4).

ДОКАЗАТЕЛЬСТВО ПРЕДЛОЖЕНИЯ 2.7.3. Утверждение следует из 2.7.6(1) и

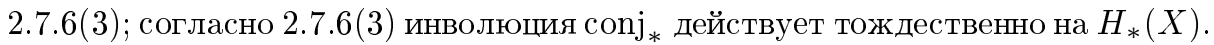

\section{8. Дополнительные запреты.}

2.8.1. ОБОБЩЕННОЕ НЕРАВЕНСТВо АРНОЛЬДА. Пусть $X$ - неособое замкнутое комплексное кәлерово многообразие четной размерности $n=2 k$, наделенное вещественной структурой. Тогда число $p_{-}$ориентируемых компонент многообразия $\mathbb{R} X$ с неотрицательной эйлеровой характеристикой удовлетворяет неравенству

$$
p_{-} \leqslant \frac{1}{4}\left(b_{n}(X)-(-1)^{k} \sigma(X)\right)+\frac{1}{2} \sum_{j=1}^{k}(-1)^{j} h^{k-j, k-j}(X)+\frac{1}{2}\left((-1)^{k}-1\right) .
$$

ДокАЗАТЕльСтво. Это неравенство получается точно так же, как в [2], из неравенства $p_{-} \leqslant \sigma_{\epsilon}^{+1}$, где $\epsilon=(-1)^{k+1}$, а $\sigma_{ \pm}^{+1}-$ индексы инерции формы пересечений многообразия $X$, суженной на $H_{n}^{+1}(X ; \mathbb{Z})$. Для нахождения $\sigma_{\epsilon}^{+1}$ воспользуемся равенствами

$$
\begin{aligned}
& \sigma_{+}^{+1}+\sigma_{-}^{+1}+\sigma_{+}^{-1}+\sigma_{-}^{-1}=b^{n}(X), \\
& \sigma_{+}^{+1}-\sigma_{-}^{+1}+\sigma_{+}^{-1}-\sigma_{-}^{-1}=\sigma(X) \\
& \sigma_{+}^{+1}-\sigma_{-}^{+1}-\sigma_{+}^{-1}+\sigma_{-}^{-1}=(-1)^{k} \chi(\mathbb{R} X), \\
& \sigma_{+}^{+1}+\sigma_{-}^{+1}-\sigma_{+}^{-1}-\sigma_{-}^{-1}=\operatorname{Trace}\left(\operatorname{conj}^{*}, H^{n}(X ; \mathbb{Z})\right) .
\end{aligned}
$$

В правой части последнего равенства группу $H^{n}(X ; \mathbb{Z})$ можно заменить на $H^{k, k}(X)$, и мы получим оценку для $\sigma_{\epsilon}^{+1}$, сравнив

$$
\operatorname{Trace}\left(\operatorname{conj}^{*}, H^{k, k}(X)\right)=\sum_{j=0}^{k}(-1)^{j} \operatorname{Trace}\left(\operatorname{conj}^{*}, P^{k-j, k-j}(X)\right)
$$

с (2.4.2) и использовав очевидное неравенство

$$
\text { - Trace }\left(\operatorname{conj}^{*}, P^{i, i}(X)\right) \leqslant \operatorname{dim} P^{i, i}(X)=h^{i, i}(X)-h^{i-1, i-1}(X) .
$$

(При нечетном $k$ можно также воспользоваться тем, что - Trace $\left(\right.$ conj $\left.^{*}, P^{0,0}(X)\right)$ дает вклад в $\sigma_{\epsilon}^{+1}$, и тем, что conj ${ }^{*}=$ id на $P^{0,0}(X)$.) 
2.8.2. Для подмножества $S \subset \mathbb{R P}^{N}$ положим

$$
\ell(S)=\max \left\{-1, i \mid \text { in }^{*}: H^{i}\left(\mathbb{R P}^{N}\right) \rightarrow H^{i}(S) \text { ненулевой }\right\}
$$

где in: $S \hookrightarrow \mathbb{R P}^{N}$ - включение. Если $A \subset \mathbb{P}^{N}$ - вещественное проективное алгебраическое многообразие, положим $\ell(A)=\ell(\mathbb{R} A)$. Ясно, что in* $H^{i}\left(\mathbb{R} \mathbb{P}^{N}\right) \rightarrow H^{i}(\mathbb{R} A)-$ ненулевой гомоморфизм для всех $i \leqslant \ell(A)$ и что $\ell(A)=n=\operatorname{dim} A$, если степень $\operatorname{deg} A$ четна. (Напомним, что степенью многообразия $A$ назьвается число его точек пересечения с общей $(N-n)$-мерной плоскостью в $\mathbb{P}^{N}$.) Следующее простое утверждение сразу следует из двойственности Пуанкаре и точности стандартных когомологических последовательностей.

2.8.3. ПРЕДЛОЖЕНИЕ. Если $\operatorname{deg} A$ четна, mо $\ell\left(\mathbb{R P}_{+}\right)=\ell(A) \leqslant \ell\left(\mathbb{R P}_{-}\right)=$ $n-l(A)$ и, в частности, $\ell(A) \leqslant \frac{n}{2}$.

ЗАмечАниЕ. Если $\operatorname{deg} A$ четна, из 2.8.3 и замечания после 2.3 .2 следует, что двулистное накрывающее пространства $\mathbb{P}^{n+1}$ есть $M$-многообразие (при соответствующем выборе вешественной структуры накрываюшего) тогда и только тогда, когда $A$ есть $(M-d)$-многообразие, где для $n$ четного

$$
d= \begin{cases}n-2 \ell(\mathbb{R} A), & \text { если } m=0 \bmod 4, \\ 1, & \text { если } m=2 \bmod 4 \text { и } n>2 \ell(\mathbb{R} A), \\ 0, & \text { если } m=2 \bmod 4 \text { и } n=2 \ell(\mathbb{R} A),\end{cases}
$$

и для $n$ нечетного

$$
d= \begin{cases}n-2 \ell(\mathbb{R} A)-1, & \text { если } m=0 \bmod 4, \\ 0, & \text { если } m=2 \bmod 4 .\end{cases}
$$

Другое следствие тех же вычислений - улучшенная оценка Смита-Тома: многообразие $A$ не может бить $(M-l)$-многообразием при $l<d$ для указанных выше $d$.

Инвариант $\ell(\mathbb{R} A)$ был введен в [59]; результаты работы [59] были позже улучшены И. О. Калининым [54].

2.8.4. ДОПОЛНИТЕЛЬНЫЕ ЭКСТРЕМАЛЬНЫЕ СРАВНЕНИЯ ДЛЯ $(M-d)$-ГИПЕРПОВеРХНОСТЕЙ. Пусть $A \subset \mathbb{P}^{n+1}$ - вещественная $(M-d)$-гиперповерхность степени $m$ и четной размерности $n$. В случае, когда $m= \pm 2 \bmod 8$ u $n / 2-\ell(A)$ нечетно,

(1) если $d=n / 2-\ell(A)$, mo $\chi(\mathbb{R} A)=\sigma(A) \mp 2 \bmod 16$;

(2) если $d=n / 2-\ell(A)+1$, mo $\chi(\mathbb{R} A)=\sigma(A), \sigma(A) \mp 4 \bmod 16$.

В случае, когда $m=0 \bmod 8$ и $\ell(A)<n / 2$,

(3) если $d=n-2 \ell(A), \operatorname{mo} \chi(\mathbb{R} A)=\sigma(A) \bmod 16$

(4) если $d=n-2 \ell(A)+1, \operatorname{mo} \chi(\mathbb{R} A)=\sigma(A) \pm 2 \bmod 16$.

ДОКАЗАТЕЛЬСТво ТЕОРЕмЫ 2.8.4 (см. [54]). Не вдаваясь глубоко в детали, мы лиш наметим основные идеи. Как и другие экстремальные сравнения, 2.8.4 выводится из 2.7.6(4) и (5) с использованием В.2.2. В решающий момент вместо 2.7.6(3) и (6) используется следующая лемма, дающая оценку для $\operatorname{dim} J=\operatorname{dim} q^{ \pm}$. 
2.8.5. Лемма (см. [54]). Пусть $A \subset \mathbb{P}^{n+1}$ - вещественная $(M-d)$-гиперповерхность размерности $n$ и степени $m$.

(1) $\operatorname{Ecлu~} \ell(A) \geqslant[(n-1) / 2]$, mo $\operatorname{dim} J=d$.

(2) $\operatorname{Ecлu~} \ell(A)<[(n-1) / 2]$, mo

$$
\operatorname{dim} J=d- \begin{cases}2([(n-1) / 2]-\ell(A)) & \text { при } m=0 \bmod 4, \\ 2[(n / 2-\ell(A)) / 2] & \text { nри } m=2 \bmod 4 \text { и } n \text { четном, } \\ (n-1) / 2-\ell(A) & \text { nри } m=2 \bmod 4 \text { и п нечетном. }\end{cases}
$$

(Здесь $[x]$ обозначает челую часть числа $x$.

Лемма 2.8.5 доказывается с использованием спектральной последовательности Калинина $\left({ }^{r} E^{*},{ }^{r} d^{*}\right)$, см. А.2. Если $A$ - регулярное полное пересечение в $\mathbb{P}^{N}$, разность $d-\operatorname{dim} J$ равна числу нетривиальных дифференциалов ${ }^{r} d^{i}$ с $r>1$. Лемма 2.8 .5 получена в [54] явньм выгислением членов ${ }^{r} E^{*}\left(\mathbb{P}^{N}\right)$ и ${ }^{r} E^{*}\left(\mathbb{P}^{n+1} \backslash A\right)$. Это вычисление дает также еше один ключевой вклад в доказательство теоремы 2.8.4 (см. следующую лемму). Обозначим через $h$ образующую группы $H^{2}\left(\mathbb{P}^{N}\right)$ и положим $\delta_{h}(A)=0$, если $n=\operatorname{dim} A$ нечетно или $n$ четно и in* $h^{n / 2} \in \operatorname{Im}\left(1+\operatorname{conj}^{*}\right)$ в $H^{n}\left(A ; \mathbb{Z}_{2}\right)$, и $\delta_{h}(A)=1$ в противном случае.

2.8.6. Лемма (см. [54]). Пусть $A \subset \mathbb{P}^{n+1}$ - вещественная гиперповерхность четной размерности $n$ и степени $m$. При $m=2 \bmod 4$

$$
\delta_{h}(A)= \begin{cases}0, & \text { если } \ell(A)<n / 2 \text { и } \ell(A)=n / 2-1 \bmod 2, \\ 1 & \text { в противном случае; }\end{cases}
$$

nрu $m=0 \bmod 4$

$$
\delta_{h}(A)= \begin{cases}0, & \text { если } \ell(A)<n / 2, \\ 1, & \text { если } \ell(A) \geqslant n / 2 .\end{cases}
$$

В условиях теоремы 2.8.4 из этой леммы следует, что in* $h^{n / 2} \in \operatorname{Im}\left(1+\right.$ conj $\left.^{*}\right)$. Это дает дополнительную информацию о $\operatorname{Br} q^{ \pm}$, когда $\operatorname{dim} q^{ \pm}=\operatorname{dim} J$ мала (см. В.1.1).

2.9. Структуры, наследуемые вещественной частью. В оставшейся части этого раздела мы кратко обсудим несколько конструкций, в какой-то степени обобщаюших понятие комплексной ориентации разбивающей вешественной кривой.

2.9.1. СПИНОРНЫЕ ОРИЕНТАЦИИ. Пусть $Х$ - неособое многообразие с вещественной структурой сопј. Тогда любая сопј-инвариантная спинорная структура на $X$ естественным образом определяет полуориентачию (т.е. пару противоположсных ориентаций) на $\mathbb{R} X$.

ЗАмЕчАниЕ. Если $X$ спинорное и $H_{1}(X)=0$, то единственная спинорная структура на $X$, очевидно, сопј-инвариантна и потому $\mathbb{R} X$ имеет каноническую полуориентацию. В частности, $\mathbb{R} X$ ориентируемо (cp. 3.4.2), что справедливо и в более широком случае инволюций на произвольных многообразиях и известно как теорема Эдмондca $[31]$. 
Конструкция. Сравним ориентации в двух точках $x_{1}, x_{2} \in \mathbb{R} X$. Соединим $x_{1}, x_{2}$ путем $\gamma$ в $X$, представим ориентации в них касательными $n$-реперами $\Xi_{1}, \Xi_{2}$ (где $\left.n=\operatorname{dim}_{\mathbb{C}} X=\operatorname{dim}_{\mathbb{R}} \mathbb{R} X\right)$, продолжим $\left(\Xi_{1}, \sqrt{-1} \Xi_{1}\right)$ и $\left(\Xi_{2}, \sqrt{-1} \Xi_{2}\right)$ до поля $2 n$-реперов $\left(\xi_{1}, \ldots, \xi_{2 n}\right)$ на $\gamma$ и найдем значение выбранной спинорной структуры на петле $\gamma \circ \operatorname{conj}_{*} \gamma$, где conj $_{*} \gamma$ оснащена полем реперов

$$
\left(\operatorname{conj}^{*} \xi_{1}, \ldots, \operatorname{conj}^{*} \xi_{n},-\operatorname{conj}^{*} \xi_{n+1}, \ldots,-\operatorname{conj}^{*} \xi_{2 n}\right) .
$$

Будем считать эти ориентации согласованньми, если в результате получится 0. Прямая проверка показывает, что если спинорная структура conj-инвариантна, результат не зависит от сделанного выбора.

2.9.2. Штифелевы ориентации. Напомним, что ориентацию гладкого многообразия $Y$ можно определить как гомотопический класс поднятий в $B S O$ классифицирующего отображения $f_{Y}: Y \rightarrow B O$ касательного расслоения многообразия $Y$. Расслоение $B S O \rightarrow B O$ можно, в свою очередь, рассматривать как $K\left(\mathbb{Z}_{2}, 0\right)$-расслоение, убивающее $w_{1} \in H^{1}(B O)$. Обобщая этот подход, можно зафиксировать характеристический класс $\omega \in H^{i+1}(B O)$ и определить $\omega$-структуру на $Y$ как гомотопический класс поднятий отображения $f_{Y}$ в пространство $K\left(\mathbb{Z}_{2}, i\right)$-расслоения $B O_{\omega} \rightarrow$ $B O$, убиваюшего $\omega$. Легко проверить, что $Y$ допускает $\omega$-структуру тогда и только тогда, когда $\omega(Y)=0$, и что для непустого $Y$ множество $\omega$-структур на $Y$ является аффинным пространством над $H^{i}(Y)$ (см., например, [24]). Таким образом, ориентации и спинорные (точнее, $\mathrm{Pin}^{+}-$) структуры являются соответственно $w_{1}$ - и $w_{2}$-структурами.

Если $\omega=w_{i+1}-$ класс Штифеля-Уитни, можно заменить $B O_{\omega} \rightarrow B O$ соответствующим ассоциированным расслоением Штифеля и показать, что $w_{i}$-структуру можно рассматривать как $\mathbb{Z}_{2}$-значную функцию на гомотопических классах $i$-циклов, оснащенных полями $(n-i)$-реперов (где $n=\operatorname{dim} Y)$. Это приводит к следующему обобшению понятия комплексной ориентации разбивающей кривой (см. [120] или более формальньй подход - в [23]). Пусть $X$ - неособое комплексное многообразие с вешественной структурой, $\operatorname{dim} X=n$ и $[\mathbb{R} X]$ равно нулю в $H_{n}(X)$. Тогда $\mathbb{R} X$ обладает канонической $w_{n}$-структурой, значение которой на $(n-1)$-цикле $\gamma$, оснашенном полем 1-реперов, определяется как коэффициент зацепления с $\mathbb{R} X$ цикла $\gamma$, сдвинутого вдоль умноженного на $\sqrt{-1}$ оснашения. (Точнее, это частичная структура, определенная на ядре гомоморфизма включения $H_{n-1}(\mathbb{R} X) \rightarrow H_{n-1}(X)$.) Условие $[\mathbb{R} X]=0$ обеспечивает корректность определения коэффициента зацепления. Эта конструкция допускает дальнейшее обобшение. Пусть $\gamma$ есть $(n-i)$-цикл и $\left(\xi_{1}, \ldots, \xi_{i}\right)$ - его оснащение. Действуя так же, как при геометрическом построении гомоморфизмов Виро (см. А.2), включим $\gamma$ в $(n-1)$-цикл $\gamma^{\prime}$, лежаший в $X$ и касаюшийся оснашения $\sqrt{-1} \xi_{1}, \ldots, \sqrt{-1} \xi_{i-1}$; затем сдвинем $\gamma^{\prime}$ вдоль некоторого продолжения поля $\sqrt{-1} \xi_{i}$ и вычислим коэффишиент зацепления сдвинутого цикла с $\mathbb{R} X$. Детальньй анализ показьвает, что эта конструкция дает частичную $w_{n-i+1}$-структуру на $\mathbb{R} X$, определенную на ядре гомоморфизма $\mathrm{bv}_{n-1}: H_{n-i}(\mathbb{R} X) \rightarrow{ }^{i} E_{n-1}(X)$ при условии, что $\mathrm{bv}_{n+i-1}[\mathbb{R} X]$ равен нулю в ${ }^{i} E_{n+i-1}(X)$. В частности, из последнего условия следует, что

$$
w_{n}(X)=\cdots=w_{n-i+1}(X)=0 .
$$




\section{3. Поверхности}

3.1. Основные результаты. В этом разделе под комплексной поверхностью мы подразумеваем неособое замкнутое комплексно-аналитическое двумерное многообразие. Сначала мы переформулируем некоторые результаты раздела 2 в более топологических терминах.

3.1.1. ТЕОРЕМА. Пусть $X$ - комплексная поверхность с вещественной структурой. Тогда

$$
\beta_{*}(\mathbb{R} X) \leqslant \beta_{*}(X) \quad u \quad \beta_{*}(\mathbb{R} X)=\beta_{*}(X) \bmod 2 .
$$

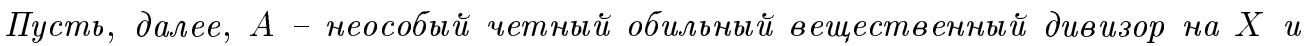
$Y \rightarrow X-$-вулистное накрытие с ветвлением над A. Тогда

$$
\begin{aligned}
\beta_{*}\left(\mathbb{R} X_{-}\right)+\beta_{*}\left(\mathbb{R} X_{+}\right) & \leqslant \beta_{2}(X)+\beta_{*}(A) \quad u \\
2 \beta_{*}\left(\mathbb{R} X_{ \pm}\right)-4 c_{0}\left(\mathbb{R} X_{ \pm}\right) & \leqslant 2 \beta_{2}(X)+\beta_{*}(A),
\end{aligned}
$$

где $\mathbb{R} X_{ \pm}$- две половинь множества $\mathbb{R} X \backslash \mathbb{R} A$ (см. 2.2) и с ${ }_{0}\left(\mathbb{R} X_{ \pm}\right)$- число замкнутых компонент поверхности $\mathbb{R} X_{ \pm}$, нетривиально накрываемых компонентами поверхности $\mathbb{R} Y_{ \pm}$.

Согласно критерию Накая-Мойшезона (см., например, [10]) эффективный дивизор $A$ на неособой кэлеровой поверхности $X$ обилен тогда и только тогда, когда $A \circ D>0$ для любой неприводимой кривой $D \subset X$. В общем случае условие обильности в 3.1.1 можно ослабить, потребовав, чтобы множество комплексных точек дивизора $A$ было связным и гомоморфизм включения $H_{1}(A) \rightarrow H_{1}(X)$ был эпиморфизмом. При этих предположениях из точной последовательности Смита следует, что $\beta_{1}(Y)=\beta_{1}(X)$.

3.1.2. ТЕОРемА. Пусть $X-$ комплексная кәлерова поверхность с вещественной структурой. Тогда

$$
|\chi(\mathbb{R} X)-1| \leqslant \sigma^{-}(X),
$$

əде $\sigma^{-}(X)=\frac{1}{2}\left[b_{2}(X)-\sigma(X)\right]$ - отрицательньи индекс инериии формы пересечений поверхности $X$. Пусть, далее, $A \subset X$ - неособый обильный четный вещественный дивизор на $X$, для которого существует вещественный дивизор $E$ на $X$ c $2 E \sim A$. Тогда

$$
\begin{gathered}
\left|\chi\left(\mathbb{R} X_{-}\right)-\chi\left(\mathbb{R} X_{+}\right)\right| \leqslant \sigma^{-}(X)-b_{1}(X)+1+\frac{1}{4}[A]^{2}-\frac{1}{2} \chi(A) \\
\left|2 \chi\left(\mathbb{R} X_{ \pm}\right)-1\right| \leqslant 2 \sigma^{-}(X)-b_{1}(X)+1+\frac{1}{4}[A]^{2}-\frac{1}{2} \chi(A)
\end{gathered}
$$

Заметим, что здесь, как и во многих других утверждениях о поверхностях (по крайней мере в этом обзоре), свойство кэлеровости необходимо лишш для того, чтобы инволюция соnj ${ }_{*}$ имела нулевой след на $H_{1}(X ; \mathbb{R})$. Поэтому его можно заменить, например, условием $b_{1}(X)=0$. Из более сильного условия $\beta_{1}(X)=0$ следовало бы, кроме того, сушествование дивизора $E$, о котором говорится в формулировке. Заметим также, что в формулировке теоремы 3.1 .2 не участвует никакое накрытие; однако мы не 
знаем, остается ли утверждение справедливым, если не будет существовать вещественного двулистного накрытия с ветвлением над $A$.

Условие обильности в последних двух неравенствах можно заменить условием $b_{1}(Y)=b_{1}(X)$; в противном случае к правой части каждого неравенства надо прибавить $b_{1}(Y)-b_{1}(X)$. Заметим, что, в отличие от 2.4.1, все эти утверждения можно доказать топологически по схеме, указанной в [2]. (Для доказательства второго неравенства надо рассмотреть обе инволюции $T_{ \pm}$на $Y$ и выгислить индексы инерции $\sigma_{ \pm}^{ \pm 1, \pm 1}$ сужений формы пересечений на собственные подпространства $H_{2}^{ \pm 1, \pm 1}$ пары инволюций. Неравенство тогда будет следовать из того, что $\sigma_{-}^{ \pm 1, \mp 1} \geqslant 0$.) Более того, рассматривая различные классы, реализуемые ориентируемыми компонентами поверхностей $\mathbb{R} X$ и $\mathbb{R} Y$, можно получить усиленные неравенства и их экстремальные свойства. Вот один из примеров:

3.1.3. ТЕОРемА. Пусть $X$ - комплексная кәлерова поверхность с вещественной структурой. Тогда

$$
-\sigma^{-}(X) \leqslant 1-\chi(\mathbb{R} X) \leqslant \sigma^{-}(X)-2 p_{+},
$$

где $p_{+}$- число ориентируемых компонент поверхности $\mathbb{R} X$ с положстельной эйлеровой характеристикой. Если $A \subset X-$ неособый обильный четный вещественныи дивизор на $X$, для которого существует вещественный дивизор $E$ на $X$ $c 2 E \sim A, m o$

$$
\chi\left(\mathbb{R} X_{-}\right)-\chi\left(\mathbb{R} X_{+}\right) \leqslant \sigma^{-}(X)-b_{1}(X)+1+\frac{1}{4}[A]^{2}-\frac{1}{2} \chi(A)-2 q_{+},
$$

əде $q_{+}$- число ориентируемых компонент поверхности $\mathbb{R} Y_{+}$с положительной эйлеровой характеристикой (соответствующих, очевидно, компонентам поверхности $\mathbb{R} X_{+}$с положительной әйлеровой характеристикой, для которых сужсене проекиии накрьтия $\mathbb{R} Y_{+} \rightarrow \mathbb{R} X_{+}$есть ориентирующее двулистное накрытие).

Экстремальные свойства сравнений 2.7.1, сравнение Арнольда 2.7.2 и предложение 2.7.3 (так же как и лемма 2.7.6) переносятся на случай поверхностей без изменений. Обобщенное неравенство Арнольда 2.8.1 принимает более простую форму:

3.1.4. НЕРАВЕНСТВО АРНОЛЬДА ДЛЯ ПОВЕРХНОСТЕЙ. Пусть X - кәлерова поверхность с вещественной структурой. Тогда число р- ориентируемых компонент поверхности $\mathbb{R} X$ с отрицательной эйлеровой характеристикой удовлетворяет неравенству

$$
p_{-} \leqslant \frac{1}{2}\left(\sigma^{+}(X)-1\right),
$$

əде $\sigma^{+}(X)=\frac{1}{2}\left[b_{2}(X)+\sigma(X)\right]$.

Ниже приводится другой пример усиленного утверждения, справедливого для поверхностей (см. [89], где, фактически, доказьвается, что класс $[\mathbb{R} X] \in H_{2}(X ; \mathbb{Z})$ делится на $2^{r+1}$ ). Другие примеры усиленных экстремальных сравнений, учитьвающие дополнительные специфические свойства поверхностей, приведены в 3.4. 
3.1.5. СРАВНЕНИЕ НИКУЛИНА. Пусть $Х$ есть $M$-поверхность с вещественной структурой и с $H_{1}(X)=0$. Если поверхность $\mathbb{R} X$ ориентируема и әйлерова характеристика каждой ее компоненты кратна $2^{r}$ для некоторого $r \geqslant 1$, то $\chi(\mathbb{R} X)=0 \bmod 2^{r+3}$.

Если $X$ - регулярное полное пересечение в $\mathbb{P}^{q}$, то комплексные характеристики, участвуюшие в 3.1.1-3.1.4 (т.е. топологические инварианты многообразий $X$ и $A$ ), можно легко найти по индукции, используя 2.5.5.

3.1.6. Если поверхность $X$ является регулярным полным пересечением в $\mathbb{P}^{q}$ полистепени $\left(m_{1}, \ldots, m_{q-2}\right)$, то

$$
\begin{gathered}
b_{1}(X)=0, \quad b_{2}(X)=\chi(X)-2, \\
\chi(X)=\mu_{q-2}\left(\mu_{1}^{2}-\mu_{2}-(q+1) \mu_{1}+\frac{1}{2} q(q+1)\right), \\
\sigma(X)=-\frac{1}{3} \mu_{q-2}\left(\mu_{1}^{2}-2 \mu_{2}-q-1\right),
\end{gathered}
$$

где $\mu_{i}-i$-й әлементарньй симметрический многочлен от $m_{1}, \ldots, m_{q-2}$. Если, кроме того, $A$ - неособая кривая, высекаемая на $X$ гиперповерхностью степени $m$, mo

$$
\chi(A)=-m \mu_{q-2}\left(m+\mu_{1}-q-1\right) .
$$

3.2. Фактор-пространство по комплексному сопряжению. Специфическим свойством поверхностей является то, что множество неподвижных точек инволюции комплексного сопряжения имеет коразмерность 2. Следовательно, фактор-пространство $X /$ conj есть многообразие; более того, легко проверить, что с точностью до изотопии сушествует единственная гладкая структура на $X /$ conj такая, что проекция $X \rightarrow X /$ conj является двулистньм накрытием с ветвлением над $\mathbb{R} X$.

Полученные четырехмерные многообразия $X /$ conj образуют очень интересньй класс. С одной стороны, они тесно связаны с алгебраическими поверхностями; с другой стороны, во многом их свойства совершенно противоположны свойствам алгебраических поверхностей. По гипотезе С. Акбулата инварианты Зайберга-Виттена такого фактор-многообразия равны нулю, если положительный индекс инерции его формы пересечений не меньше 2. Сильным основанием для нее является то, что если $\mathbb{R} X$ имеет компоненту рода $\geqslant 2$, равенство нулю инвариантов сразу следует из “гладкого варианта" неравенства присоединения, примененного к образу этой компоненты в факторе (указанный вариант этого неравенства, восходящий к Кронхаймеру и Мровке, см. в [110]); для случая $\mathbb{R} X=\varnothing$ гипотеза была доказана Ш. Вонгом [123]. (Если $\mathbb{R} X=\varnothing$, проекция $X \rightarrow X /$ conj является настоящим (неразветвленным) накрытием и потому можно легко проконтролировать поведение решений уравнения Зайберга-Виттена.) Более того, во многих случаях (см. ниже) фактор-многообразие $X /$ conj вполне разложимо, т.е. представляется в виде связной суммы копий многообразий $\mathbb{P}^{2}, \overline{\mathbb{P}^{2}}$ и $S^{2} \times S^{2}$. (Напомним, что минимальные алгебраические поверхности неприводимы.) Таким образом, во многих случаях можно утверждать, что $X /$ conj не допускает ни комплексной, ни симплектической структуры. Имеется замечательное исключение: если $X$ есть $K 3$-поверхность, многообразие $X /$ conj диффеоморфно рациональной поверхности или поверхности Энриквеса, см. ниже.

Согласно Арнольду следуюший результат принадлежит Максвеллу: 


\subsection{1. Теорема. Фактор-пространство $\mathbb{P}^{2} / \operatorname{conj}$ диффеоморфно $S^{4}$.}

Приведенное ниже доказательство имеется, например, в [76]. Оно немного короче других, более прямых доказательств, опубликованных в [72], [78], [4]. (Возможно, за исключением доказательства в [78]; как объясняется в [5], это красивое явное доказательство было, по-существу, известно Максвеллу; обобшение этого утверждения на высшие размерности также дано в [5].) В основе доказательства лежит следующая теорема Серфа (см. [14]): группа диффеоморфизмов трехмерной сферы совпадает c группой диффеоморфизмов четырехмерного иара (или, короче, $\left.\Gamma_{4}=0\right)$. Главным достоинством этого доказательства является то, что его можно обобшить на другие вешественные поверхности, часто с использованием теоремы Лауденбаха-Поэнару [74], распространяюшей теорему Серфа на тела с ручками.

ДокаЗАтельство. Рассмотрим вешественный флаг $\mathbb{P}^{0} \subset \mathbb{P}^{1} \subset \mathbb{P}^{2}$. Факторизуя его по инволюции соnj, получим клеточное разбиение фактор-пространства: четырехмерньй шар приклеен к кругу. Фактор-пространство замкнутого четырехмерного шара с центром в начале координат пространства $\mathbb{P}^{2} \backslash \mathbb{P}^{1}$ есть четырехмерньй шар, а замькание его дополнения есть замкнутая регулярная окрестность круга $\mathbb{P}^{1} /$ conj. Kaк известно, замкнутая регулярная окрестность гладкого круга в гладком четырехмерном многообразии есть четырехмерный шар. Таким образом, два четырехмерных шара приклеиваются друг к другу и согласно теореме Серфа получаюшееся пространство диффеоморфно $S^{4}$.

Большинство обобщений теоремы 3.2.1 утверждает, что при некоторых условиях, наложенных на поверхность (обычно поверхность должна быть явно построена таким образом, чтобы можно было проконтролировать топологию фактора), фактор-пространство вполне разложимо. Однако имеется также несколько примеров поверхностей, фактор-пространства которых односвязны, но не вполне разложимы. Ниже мы даем краткий перечень известных результатов.

Для непустых квадрик и кубик в $\mathbb{P}^{3}$ разложимость доказал М. Летиция [75]. (Ввиду 3.2.1 раздутие вешественной точки не меняет фактор-пространство; поэтому для квадрик и связных кубик этот результат следует из 3.2.1.) Для непустых квартик и, более общо, для непустых $K 3$-поверхностей разложимость была доказана С. Дональдсоном [30]. (Любопытно, что некоторые $K 3$-поверхности, так называемые поверхности Френеля-Куммера, связаны с электромагнитной теорией Максвелла.) Подход Дональдсона основан на изменении комплексной структуры в семействе твисторов и преобразовании вешественной структуры в голоморфную инволюцию, умножающую голоморфные формы на -1 ; при этом фактор-пространство становится рациональной поверхностью, диффеоморфной, очевидно, $\mathbb{P}^{2} \# k \overline{\mathbb{P}^{2}}$ или $S^{2} \times S^{2}$.

То, что фактор является вполне разложимым, доказано для двулистных накрытий плоскости, разветвленных над кривыми степени $2 k$, вешественная часть которых состоит из единственного гнезда глубины $k$ (С. Акбулат [1]), или над кривыми, полученными мальм возмущением объединения вещественных прямых (С. Финашин [36]). Финашин распространил эти результаты на двулистные накрытия квадрик с ветвлением над малыми возмущениями объединений образующих и на некоторые регулярные полные пересечения в $\mathbb{P}^{N}$, рекурсивно получаемые возмушением объединений. Для более широкого класса плоских кривых он доказал, что факторы соответствующих 
двулистных накрытий плоскости становятся вполне разложимьми после добавления нескольких копий многообразия $\overline{\mathbb{P}^{2}}$.

Свойство фактора быть вполне разложимым справедливо для всех вешественных структур на рациональных поверхностях (доказательство основано на классификации вещественных рациональных поверхностей, см. 3.6.1) и для всех вещественных поверхностей Энриквеса (использование модифицированного трюка Дональдсона, см. конец п. 3.7, позволяет свести случай поверхностей Энриквеса к случаю рациональных поверхностей). Подробности см. в [37].

Пример комплексной поверхности с вешественной структурой, фактор которой не является вполне разложимым, построен С. Финашиным и Е. Шустиным [39]. Этот фактор является односвязным спинорным многообразием с ненулевой сигнатурой (и, следовательно, не диффеоморфным многообразию $\left.k\left(S^{2} \times S^{2}\right)\right)$. В настоящее время неизвестно, сушествуют ли поверхности, факторы которых по комплексному сопряжению не раскладьваются в связную сумму копий многообразий $\mathbb{P}^{2}, \overline{\mathbb{P}^{2}}, S^{2} \times S^{2}$ и $K 3$-поверхностей (добавление $K 3$-поверхностей позволяет получать спинорные многообразия с ненулевой сигнатурой).

3.3. Комплексное разбиение и квадратичная форма Понтрягина-Виро. Пусть $X$ - комплексная поверхность с вещественной структурой, $H_{1}(X)=0$, и пусть $A$-вешественная кривая на $X$. (Мы не исключаем случай $A=\varnothing$.) Говорят, что пара $(X, A)$ относится к характеристическому типу, если $[\mathbb{R} X]+[A]=w_{2}(X)$ в $H_{2}(X)$. Обозначим через $\mathrm{pr}: X \rightarrow X^{\prime}$ проекцию на фактор-пространство $X^{\prime}=X /$ conj. Из точности последовательности Смита для автоморфизма этого накрытия и формулы проекции $w_{2}(X)=\operatorname{pr}^{*} w_{2}\left(X^{\prime}\right)+\mathrm{D}^{-1}[\mathbb{R} X]$ следует, что rel $w_{2}\left(X^{\prime}\right)=\left[A^{\prime}\right]$ в $H_{2}\left(X^{\prime}, \mathbb{R} X\right)$ (напомним, что $A^{\prime}=A /$ conj). Таким образом, класс $[\mathbb{R} A]=\partial w_{2}\left(X^{\prime}\right)$ равен нулю в $H_{1}(\mathbb{R} X)$, и потому существует такая поверхность $\mathbb{R} X_{+}^{w} \subset \mathbb{R} X$, что $\partial \mathbb{R} X_{+}^{w}=\mathbb{R} A$, причем $\mathfrak{A}_{+}^{w}=\mathbb{R} X_{+}^{w} \cup A^{\prime}$ реализует класс $w_{2}\left(X^{\prime}\right)$. Пусть $\mathbb{R} X_{-}^{w}$ - замыкание множества $\mathbb{R} X \backslash \mathbb{R} X_{+}^{w}$. Так как $\mathbb{R} X$ гомологично нулю в $X^{\prime}$, поверхность $\mathfrak{A}_{-}^{w}=\mathbb{R} X_{-}^{w} \cup A^{\prime}$ также реализует класс $w_{2}\left(X^{\prime}\right)$. Разложение $\mathbb{R} X=\mathbb{R} X_{+}^{w} \cup \mathbb{R} X_{-}^{w}$, удовлетворяюшее этим условиям, единственно, поскольку опять же в силу точности последовательности Смита $\operatorname{dim} \operatorname{Ker}\left[H_{2}(\mathbb{R} X) \rightarrow H_{2}\left(X^{\prime}\right)\right]=1$. Оно называется комплексным разбиением пары $(X, A)$.

Так как $H_{1}(X)=0$, поверхности Арнольда $\mathfrak{A}_{ \pm}^{w}$, являясь характеристическими, обладают каноническими $\mathrm{Pin}^{-}$-структурами и соответствующими формами Рохлина-Гийю-Марена, см. приложение С. Обозначим эти формы через $\mathfrak{q}_{ \pm}^{w}$, а их сужения на $\mathbb{R} X_{ \pm}^{w}$ - через $q_{ \pm}^{w}$. Легко проверить, что $q_{ \pm}^{w}$ равны нулю на ядрегомоморфизма включения $H_{1}\left(\mathbb{R} X_{ \pm}^{w}\right) \rightarrow H_{1}(\mathbb{R} X)$.

Следующий результат принадлежит Г.Б. Михалкину [81].

3.3.1. ЛЕмма. При сделанных предположениях

$$
\chi\left(\mathbb{R} X_{-}^{w}\right)=\frac{1}{4}[\chi(\mathbb{R} X)-\sigma(X)]+\frac{1}{2}[A]^{2}+\operatorname{Br} \mathfrak{q}_{-}^{w} \bmod 8
$$

3.3.2. Теорема. Для указанньх выше $X, A$ пусть форма $q_{-}^{w}$ равна нулю на образе группь $H_{1}(\mathbb{R} A)$. Положим $R=\frac{1}{4}\left(\chi(\mathbb{R} X)-\sigma(X)+[A]^{2}\right)+\operatorname{Br} q_{-}^{w}$.

(1) Если $A$ есть $M$-кривая, то $\chi\left(\mathbb{R} X_{-}^{w}\right)=R \bmod 8$.

(2) Если $A$ есть $(M-1)$-кривая, то $\chi\left(\mathbb{R} X_{-}^{w}\right)=R \pm 1 \bmod 8$. 
(3) Если $A$ есть $(M-2)$-кривая и $\chi\left(\mathbb{R} X_{-}^{w}\right)=R+4 \bmod 8$, mо A принадлежит muny I.

(4) Если А принадлежит типу I, то $\chi\left(\mathbb{R} X_{-}^{w}\right)=R \bmod 4$

Заметим, что лемма 3.3.1 и теорема 3.3.2 имеют топологическую природу и потому справедливы для гибких кривых на гибких поверхностях; в последнем случае $A$ может быть даже неориентируемой.

ДоКАЗАТЕЛЬСТВо УТВЕРЖДЕНИЙ 3.3.1 и 3.3.2. Лемма 3.3.1 следует из сравнения Рохлина-Гийю-Марена [46] и непосредственного вычисления индекса пересечения: $\left[\mathfrak{A}_{-}^{w}\right] \circ\left[\mathfrak{A}_{-}^{w}\right]=\frac{1}{2}[A]^{2}-2 \chi\left(\mathbb{R} X_{-}^{w}\right)$. Доказательство теоремы 3.3.2 повторяет одно из доказательств классического экстремального сравнения для плоских кривых (см. [77]); оно основано на лемме 3.3.1, аддитивности инварианта Брауна и следуюших очевидных фактах: если $A$ есть $(M-d)$-кривая, то $\operatorname{dim} H_{1}\left(A^{\prime}\right)=d$; поверхность $A^{\prime}$ ориентируема тогда и только тогда, когда $A$ принадлежит типу I.

Следуюшее предложение сразу следует из определения формы Рохлина-Гийю-Марена и теоремы 3.3.2:

3.3.3. ПРЕДЛОЖЕНИЕ. Сужсения форм $\mathfrak{q}_{ \pm}^{w}$ на $H_{1}\left(A^{\prime}\right)$ отличаются друг от друга на $2 \omega$, где $\omega$ - характеристический класс накрытия $A \backslash \mathbb{R} A \rightarrow A^{\prime} \backslash \mathbb{R} A$. Следовательно, формы $q_{ \pm}^{w}$ могут обращаться в нуль на образе группьи $H_{1}(\mathbb{R} A)$ только одновременно, и в этом случае

$$
\operatorname{Br} q_{+}^{w}+\operatorname{Br} q_{-}^{w}=\frac{1}{2}\left(\chi(\mathbb{R} X)+\sigma(X)-[A]^{2}\right) \bmod 8
$$

а если А принадлежит типу I, то

$$
\chi\left(\mathbb{R} X_{+}^{w}\right)-\chi\left(\mathbb{R} X_{-}^{w}\right)=\operatorname{Br} q_{+}^{w}-\operatorname{Br} q_{-}^{w} \bmod 8 .
$$

(Для гибких кривых в первом сравнении А долэсна быть ориентируемой; второе сравнение справедливо и для неориентируемой кривой А.)

Указанные сужения $q_{ \pm}^{w}$ можно вычислить, используя спектральную последовательность Калинина, см. А.2. Покажем, что из двух форм $q_{ \pm}^{w}$ можно получить одну квадратичную форму на $H_{1}(\mathbb{R} X)$, которая зависит только от топологии поверхности $(X$, conj $)$ и от класса $[A] \in H_{2}(X ; \mathbb{Z})$.

Зафиксируем такой класс $c \in H_{2}(X ; \mathbb{Z})$, что $\operatorname{conj}_{*} c=-c$ и $c=[\mathbb{R} X]+w_{2}(X) \bmod 2$. Выберем $x \in H_{1}(\mathbb{R} X ; \mathbb{Z})$ и найдем такую целочисленную цепь $x^{\prime}$ в $X$, что $\partial x^{\prime}=x$. (При этом может оказаться необходимьм умножить цикл $x$ на нечетное число, что не влияет на дальнейшее, поскольку предполагается лишш, что $H_{1}(X)=0$.) Легко проверить, что $\left(1-\operatorname{conj}_{*}\right) x^{\prime}$ - целочисленньй цикл и что вычет

$$
\mathscr{P}_{c}(x)=\left[\left(1-\operatorname{conj}_{*}\right) x^{\prime}\right]^{2}+c \circ\left(1-\operatorname{conj}_{*}\right) x^{\prime} \bmod 4
$$

не зависит от выбора цепи $x^{\prime}$. Более того, отображение $x \mapsto \mathscr{P}_{c}(x)$ есть квадратичное расширение формы пересечений $(x, y) \mapsto x \circ y$; к тому же так как $c$ преврашается в 0 в ${ }^{\infty} E_{2}$, то $\mathscr{P}_{c}(-x)=\mathscr{P}_{c}(x)+2\left(c \circ \mathrm{bv}_{2} x\right)=\mathscr{P}_{c}(x)$ и, значит, $\mathscr{P}_{c}$ пропускается через $H_{1}(\mathbb{R} X)$. Функция $\mathscr{P}_{c}: H_{1}(\mathbb{R} X) \rightarrow \mathbb{Z}_{4}$ назьвается квадратичной формой Понтрягина-Виро. 
3.3.4. ПРЕДЛОЖЕНИЕ. Справедливы следующие утверждения:

(1) $\mathscr{P}_{c+2 e}(x)=\mathscr{P}_{c}(x)+2\left(e \circ \mathrm{bv}_{2} x\right)$ для любого $e \in H_{2}(X ; \mathbb{Z}) c \operatorname{conj}_{*} e=-e$;

(2) $\operatorname{Br} \mathscr{P}_{c}=\frac{1}{2}\left[\chi(\mathbb{R} X)+\sigma(X)-c^{2}\right] \bmod 8$;

(3) если $A$ - такая (әибкая) вещественная кривая на $X$, что пара $(X, A)$ принадлежит характеристическому типу, то q формиц $\mathscr{P}_{[A]}$ на $\mathbb{R} X_{ \pm}^{w}$.

ДокАЗАТЕльство. (1) сразу следует из определения; (3) доказывается непосредственным вычислением индексов перепонок. Чтобы доказать (2), достаточно построить такую ориентируемую гибкую кривую $A$ на $X$, что пара $(X, A)$ принадлежит характеристическому типу и $\mathscr{P}_{[A]}$ равно нулю на $\mathbb{R} A$ (или $\mathbb{R} A=\varnothing$ ); утверждение для $\mathscr{P}_{[A]}$ тогда будет следовать из (3) и предложения 3.3.3, и его можно распространить на другие значения $c$, используя (1) и В.1.5(3). Такую кривую можно легко построить: поскольку $[\mathbb{R} X]+w_{2}(X)=0$ в ${ }^{\infty} E_{2}$, существует такой класс $f \in H_{2}(X ; \mathbb{Z})$, что $\left(1-\operatorname{conj}_{*}\right) f=[\mathbb{R} X]+w_{2}(X) \bmod 2 H_{2}(X ; \mathbb{Z})$. Реализуем $f$ гладкой ориентируемой поверхностью $F$, трансверсальной к $\mathbb{R} A$, и возмутим особенности поверхности $F \cup(-\operatorname{conj} F)$ в согласии с ориентацией.

Упомянем о двух специальных случаях формы Понтрягина-Виро. Целочисленное поднятие $c$ класса $w_{2}(X)$ соответствует $\operatorname{Spin}^{\mathbb{C}}$-структурена $X$. Поэтому если $[\mathbb{R} X]=0$ в $H_{2}(X)$, соответствие $c \mapsto \mathscr{P}_{c}$ позволяет для любой $\operatorname{Spin}^{\mathbb{C}}$-структуры на $X$ получить $\mathrm{Pin}^{-}{ }^{-}$-структуру на $\mathbb{R} X$ (ср. 2.9.2; это соответствие можно продолжить на все, не обязательно косоинвариантные относительно conj $_{*}$, классы $c$, используя $3.3 .4(1)$ ). Подобное соответствие имеется также в случае, когда поверхность $\mathbb{R} X$ ориентируема, но не обязательно гомологична нулю; оно имеет вид $c \mapsto \mathscr{P}_{c-[\mathbb{R} X]}$, где $[\mathbb{R} X]-$ целочисленньй фундаментальньй класс поверхности $\mathbb{R} X$, наделенной некоторой ориентацией. (Ввиду 3.3.4(1) это отображение не зависит от выбранной ориентации, поскольку ориентируемые компоненты поверхности $\mathbb{R} X$ аннулируют $\mathrm{bv}_{2} H_{1}(\mathbb{R} X)$.)

Другой специальньй случай, о котором стоит упомянуть, - когда поверхность $\mathbb{R} X$ сама является характеристической в $X$ (и, следовательно, $[A]=0 \bmod 2 H_{2}(X ; \mathbb{Z})$ ). В этом случае можно взять $c=0$, тогда полученная квадратичная форма $\mathscr{P}=\mathscr{P}_{0}$ есть не что иное, как форма Рохлина-Гийю-Марена на $\mathbb{R} X$. Имеется другой способ построения формы $\mathscr{P}$, объясняюший ее название: легко проверить, что в силу равенства $[\mathbb{R} X]=w_{2}(X)$ квадрат Понтрягина $P_{2}: H_{2}(X) \rightarrow \mathbb{Z}_{4}$ можно спустить в ${ }^{2} E_{2}={ }^{\infty} E_{2}$ и положить $\mathscr{P}=P_{2} \circ \mathrm{bv}_{2}: H_{1}(\mathbb{R} X) \rightarrow \mathbb{Z}_{4}$. В таком виде эта конструкция допускает обобшения на неоднородные классы в $H_{*}(\mathbb{R} X)$ и на многообразия большей размерности, не обязательно односвязные. А именно, пусть $X$ - замкнутое комплексное многообразие с вешественной структурой, и пусть $\operatorname{dim} X=2 n$. (На самом деле, большинство утверждений можно модифицировать так, чтобы они стали справедливьми для любого замкнутого многообразия с сохраняющей ориентацию инволюцией, размерность которого делится на 4; подробности можно найти в [25] или [29].) Предположим, что квадрат Понтрягина $P_{2 n}: H_{2 n}(X) \rightarrow \mathbb{Z}_{4}$ спускается в ${ }^{\infty} E_{2 n}(X)$, и определим форму Понтрягина-Виро, положив $\mathscr{P}=P_{2 n} \circ \mathrm{bv}_{2 n}: \mathscr{F}^{2 n} \rightarrow \mathbb{Z}_{4}$. При таком определении форма $\mathscr{P}$ является квадратичным расширением гомологического спаривания Калинина $*: \mathscr{F}^{2 n} \otimes \mathscr{F}^{2 n} \rightarrow \mathbb{Z}_{2}$.

Следующие утверждения доказаны в [25] (см. также [29]). 
3.3.5. ПРЕДЛОЖЕНИЕ. Форма Понтрягина-Виро определена тогда и только тогда, когда класс Ву $u_{2 n}\left(X^{\prime} \backslash \mathbb{R} X\right)$ равен нулю. $B$ этом случае $\operatorname{Br} \mathscr{P}=\sigma(X)$ $\bmod 8$.

3.3.6. ПРЕДЛОЖЕНИЕ. Квадрат Понтрягина $P_{2 n}$ спускается в ${ }^{2} E_{2 n}$ тогда и только тогда, когда $[\mathbb{R} X]$ реализует класс Ву $u_{2 n}(X)$. Таким образом, равенство $[\mathbb{R} X]=u_{2 n}(X)$ является необходимым условием возможсности определить форму Я. Следующие условия являются достаточными:

(1) $Х$ есть $М$-многообразие;

(2) $[\mathbb{R} X]=u_{2 n}(X)$ и $X$ является $\mathbb{Z}_{2}$-Галуа максимальнылм (см. А.1.4);

(3) $[\mathbb{R} X]=u_{2 n}(X)$ u $H_{i}(X)=0$ для $0<i<2 n$.

Пусть теперь $n=1$, т.е. $X$ - поверхность. Условие $u_{2}\left(X^{\prime} \backslash \mathbb{R} X\right)=0$ в 3.3 .5 равносильно требованию: класс $u_{2}\left(X^{\prime}\right)=w_{2}\left(X^{\prime}\right)$ лежит в образе гомоморфизма включения $H_{2}(\mathbb{R} X) \rightarrow H_{2}\left(X^{\prime}\right)$, т.е. $\mathbb{R} X$ содержит поверхность, которая в $X^{\prime}$ является характеристической. Такая поверхность $F \subset \mathbb{R} X$ характеризуется свойством: $\mathscr{P}(x)=$ $2([F] \circ x) \bmod 4$ для всех $x \in \mathscr{F}^{2} \cap H_{0}(\mathbb{R} X)$. Возьмем такую $F$, обозначим через $H_{F}$ образ групшы $\mathscr{F}^{2}$ при естественном отображении $\mathscr{F}^{2} \hookrightarrow H_{*}(\mathbb{R} X) \rightarrow H_{1}(\mathbb{R} X) \rightarrow H_{1}(F)$ и определим квадратичную функцию $\mathscr{P}_{F}: H_{F} \rightarrow \mathbb{Z}_{4}$ следующим образом:

$$
x_{1} \mapsto \mathscr{P}\left(x_{1}+x_{0}\right)+2\left([F] \circ x_{0}\right),
$$

где $x_{0} \in H_{0}(\mathbb{R} X)$ - любой элемент, для которого $x_{1}+x_{0} \in \mathscr{F}^{2}$.

3.3.7. ПРЕДЛОЖЕНИЕ. Функиия $\mathscr{P}_{F}: H_{F} \rightarrow \mathbb{Z}_{4}$ совпадает с формой РохлинаГийю-Марена характеристической поверхности $F \subset X^{\prime}$. В частности, $H_{F}$ совпадает с ядром гомоморфизма включения $H_{1}(F) \rightarrow H_{1}\left(X^{\prime}\right)$, подпространство $H_{F} \subset H_{1}(F)$ информативно (относительно $\mathscr{P}_{F}$, определение информативности см. в B.1) $и$

$$
\chi(F)=\frac{1}{4}[\chi(\mathbb{R} X)-\sigma(X)]+\operatorname{Br} \mathscr{P}_{F} \bmod 8 .
$$

Если, кроме того, $H_{1}(X)=0$, из последнего утверждения следует $3.3 .4(3)$ (для $A=\varnothing)$; более того, в этом случае можно проверить, что комплексное разбиение поверхности $\mathbb{R} X$, определенное в 3.3 , можно выразить в терминах формы $\mathscr{P}:$ компоненты $C_{1}, C_{2}$ поверхности $\mathbb{R} X$ лежат обе в $\mathbb{R} X_{+}^{w}$ или обе в $\mathbb{R} X_{-}^{w}$ тогда и только тогда, когда $\mathscr{P}\left(\left\langle C_{1}-C_{2}\right\rangle\right)=0$.

3.4. Спинорные сравнения. Ниже мы приводим два экстремальных сравнения, которые связаны с $w_{2}(X)$ и ориентируемостью поверхности $\mathbb{R} X$. Другие подобные результаты, использующие более тонкую информацию о гомотопическом типе многообразия $X$, можно найти в 3.7 (см. также монографию [29], посвященную детальному изучению вешественных поверхностей Энриквеса; фактически, приведенные ниже неравенства являются побочным продуктом результатов и техники, развитой в [29]).

3.4.1. ЭКСТРЕМАЛЬНОЕ СРАВНЕНИЕ ДЛЯ НЕСПИНОРНЫХ ПОВЕРХНОСТЕЙ.

Пусть $X$ - комплексная замкнутая поверхность с вещественной структурой, причем $w_{2}(X) \neq 0$.

(1) Если $X$ есть $M$-поверхность, то $\mathbb{R} X$ неориентируема.

(2) Eсли $w_{2}(X)$ принадлежсит образу группь Tors $H_{2}(X ; \mathbb{Z})$ в $H_{2}(X)$ u $\mathbb{R} X$ ориентируема, то $X$ есть $(M-d)$-поверхность, $d \geqslant 2 u$ 
$-\chi(\mathbb{R} X)=\sigma(X) \bmod 16$ nри $d=2$;

$-\chi(\mathbb{R} X)=\sigma(X) \pm 2 \bmod 16$ npu $d=3$;

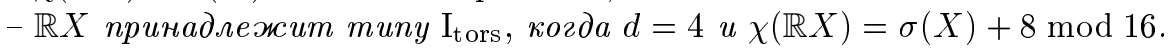

3.4.2. ЭКСТРЕМАЛЬНОЕ СРАВНЕНИЕ ДЛЯ СПИНОРНЫХ ПОВЕРХНОСТЕЙ. Пусть $X$ - комплексная замкнутая поверхность с вещественной структурой, причем $w_{2}(X)=0$.

(1) Eсли $H_{1}(X)=0$, то $\mathbb{R} X$ ориентируема.

(2) Eсли $b_{1}(X)=0$ u $\mathbb{R} X$ неориентируема, то $X$ есть $(M-d)$-поверхность, $d \geqslant 2 u$

$-\chi(\mathbb{R} X)=\sigma(X) \bmod 16$ npu $d=2$;

$-\chi(\mathbb{R} X)=\sigma(X) \pm 2 \bmod 16$ npu $d=3$;

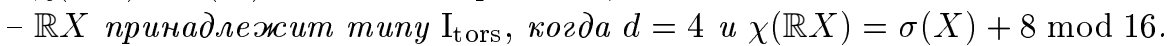

Для доказательства сравнений (утверждения (2) в 3.4 .2 и в 3.4.1) покажем, что часть групшы $T_{2}(X)=$ Tors $H_{2}(X ; \mathbb{Z}) \otimes \mathbb{Z}_{2} \subset H_{2}(X)$ преврашается в 0 в ${ }^{\infty} E_{2} ;$ тогда получим, что $\operatorname{dim} J<d$, откуда и будут следовать сравнения (cp. 2.7).

ДОКАЗАТЕЛЬСТво УТВЕРЖДЕНИЯ 3.4.1. Класс $w_{2}(X) \neq 0$ преврашается в 0 в ${ }^{\infty} E_{2}$ тогда и только тогда, когда $\mathbb{R} X$ ориентируема (см. табл. 4 в A.2.6), поэтому утверждение доказано.

ДОКАЗАТЕЛЬСТвО УТВЕРЖДЕНИЯ 3.4.2. (1) Если сушествует $x_{1} \in H_{1}(\mathbb{R} X)$ с $x_{1}^{2}=1$, то $\left(\mathrm{bv}_{2} x_{1}\right)^{2}=1$. (Kласc $\mathrm{bv}_{2} x_{1}$ определен, так как $H_{1}(X)=0$.) Это противоречит предположению о том, что форма пересечений в $H_{2}(X)$ и, следовательно, в ${ }^{\infty} E_{2}$ четна.

(2) По предположению существует $x_{1} \in H_{1}(\mathbb{R} X ; \mathbb{Z})$ с $x_{1}^{2}=1$. Как и в случае (1), заключаем, что bv $x_{1} \notin \mathrm{bv}_{1} H_{0}(\mathbb{R} X)$. Более того, любая неориентируемая компонента $C_{i}$ поверхности $\mathbb{R} X$ имеет четный род (иначе $\left[C_{i}\right]^{2}=1 \bmod 2$ ). Поэтому bv $x_{1} \notin \mathrm{Sq}_{1}{ }^{\infty} E_{2}$ и, следовательно, $H_{2}(X) / H_{2}(X ; \mathbb{Z}) \otimes \mathbb{Z}_{2}$ не содержится в $\operatorname{Im} b_{2}$.

ЗАмечАнИЕ. Утверждение 3.4.2(1) можно также получить из 2.9.1: если $H_{1}(X)=0$, то единственная спинорная структура на $X$, очевидно, conj-инвариантна, и конструкция, указанная в 2.9.1, дает каноническую полуориентацию поверхности $\mathbb{R} X$.

3.5. Поверхности в $\mathbb{P}^{3}$. В нескольких следующих пунктах мы приводим известные результаты по классификации поверхностей. Для описания топологического типа вешественной части поверхности обозначим через $S_{p}, p \geqslant 0$, ориентируемую замкнутую поверхность рода $p: S_{p}=\#_{p}\left(S^{1} \times S^{1}\right)$, а через $V_{q}, q \geqslant 1,-$ неориентируемую замкнутую поверхность рода $q / 2: V_{q}=\#_{q} \mathbb{R P}^{2}$ (в последнем случае см. наше определение рода в 4.1). Положим $S=S_{0}$.

Поверхности в $\mathbb{P}^{3}$ с топологической точки зрения традиционно изучаются с точностью до следуюших отношений эквивалентности: гомеоморфность вещественных частей поверхностей, объемлющая изотопность вешественных частей в $\mathbb{R} \mathbb{P}^{3}$, жесткая изотопность (т.е. изотопность в классе неособых или, более общо, эквисингулярных в некотором подходящем смысле поверхностей фиксированной степени) и, наконец, грубая проективная эквивалентность (т.е. эквивалентность с точностью до проективных преобразований и жестких изотопий). Два последних отношения отличаются друг 
от друга из-за того, что группа $P G L(4 ; \mathbb{R})$ проективных преобразований пространства $\mathbb{R P}^{3}$ имеет две компоненты связности. Ясно, что преобразования, содержащиеся в одной компоненте с тождественньм, переводят поверхность в жестко изотопную.

Для любой степени число классов эквивалентности конечно (для каждого из четырех отношений); это следует из того, что для каждого отношения универсальное семейство гиперповерхностей данной степени допускает такую конечную (полуалгебраическую) стратификацию, что относительно выбранного отношения это семейство локально тривиально над каждым стратом. (Для топологического отношения эквивалентности существование стратификации было доказано А. Уоллесом [122] и А.Н. Варченко [114].)

Ясно, что вешественная поверхность в $\mathbb{P}^{3}$ степени 1 есть вешественная проективная плоскость. Классификация поверхностей степени 2 - предмет классической аналитической геометрии. С точностью до жесткой изотопии имеется восемь типов таких поверхностей (включая особые), различаюшихся топологией своих вешественных частей. Неособая вешественная поверхность $X \subset \mathbb{P}^{3}$ степени 2 - это гиперболоид $\left(\mathbb{R} X=S_{1}\right)$, эллипсоид $(\mathbb{R} X=S)$ или пустая поверхность $(\mathbb{R} X=\varnothing)$. Заметим, что неособая квадрика $X$ изоморфна $\mathbb{P}^{1} \times \mathbb{P}^{1}$ и как абстрактная поверхность имеет четыре неэквивалентные вещественные структуры: имеются две вещественные структуры с пустой вешественной частью.

В рамках этого обзора мы рассматриваем только неособые поверхности. Для таких поверхностей полная классификация (для каж дого из четырех отношений эквивалентности) известна для степеней $\leqslant 4$. Для степеней $\geqslant 5$ неизвестно даже максимальное число компонент связности вешественной части поверхности. (Заметим, что максимум всегда может быть реализован неособой поверхностью.) Наилучшая из известных верхних границ получается объединением неравенств Смита и Комессатти (Петровского-Олейник). Стоит упомянуть, что каждое из этих неравенств в отдельности является точным (см. [118], где доказано также, что усиленное неравенство Комессатти $1-\chi(\mathbb{R} X) \leqslant \sigma^{-}(X)-2 p_{+}$является точным). Однако имеются основания считать, что полученные из них границы для $\beta_{0}$ и $\beta_{1}$ не точны. Асимптотически $\max \beta_{i} \sim k_{i} d^{3}$, $i=0,1$, где $d$ - степень, $\frac{13}{36} \leqslant k_{0} \leqslant \frac{5}{12}$ и $\frac{13}{18} \leqslant k_{1} \leqslant \frac{5}{6}$ (примеры, устанавливающие нижние границы, построены $\Phi$. Бианом [11]).

3.5.1. Вещественные кубические поверхности. Классификация вешественных поверхностей степени 3 в $\mathbb{P}^{3}$ получена Л. Шлефли [105], Ф. Клейном [67] и И. Г. Цейтеном [127].

3.5.2. Имеется пять жестких изотопических типов неособых вещественных кубических поверхностей в $\mathbb{P}^{3}$. Они отличаются вещественными частями: $V_{7}$, $V_{5}, V_{3}, V_{1} \sqcup S u V_{1}$.

ДокАЗАТЕЛЬСТво. Топологические инварианты комплексной части неособой кубической поверхности $X \subset \mathbb{P}^{3}$ указаны в 3.1.6: $\beta_{*}(X)=9, \sigma(X)=-4$ и $\sigma^{-}(X)=5$. Следовательно, топологические типы вещественной части, перечисленные в $3.5 .2,-$ это все типы, не запрещаемые неравенством Смита 3.1.1, экстремальным сравнением 2.7.1 и неравенством Комессатти-Петровского 3.1.2. Поэтому остается показать, что все пять топологических типов реализуются и что пространство неособых кубик имеет не более пяти компонент связности. Рассмотрим пространство $\mathbb{R} \mathscr{C}_{3}=\mathbb{R} \mathbb{P}^{19}$ всех вешественных кубических поверхностей в $\mathbb{P}^{3}$. Пусть $\mathbb{R} \Delta \subset \mathbb{R} \mathscr{C}$ - множество особых 
кубик, $\Delta_{0} \subset \mathbb{R} \Delta-$ множество кубик с единственной невырожденной двойной точкой, и пусть $\mathscr{S}=\mathbb{R} \Delta \backslash \Delta_{0}$. Тогда $\operatorname{dim} \mathbb{R} \Delta=18$ и $\operatorname{dim} \mathscr{S} \leqslant 17$ и из двойственности Пуанкаре и точности гомологической последовательности тройки $\left(\mathbb{R} \mathscr{C}_{3}, \mathbb{R} \Delta, \mathscr{S}\right)$ следует, что $\operatorname{dim} H^{0}\left(\mathbb{R} \mathscr{C}_{3} \backslash \mathbb{R} \Delta\right)=1+\operatorname{dim} \operatorname{Kerin}{ }_{*}$, где in ${ }_{*}: H_{18}(\mathbb{R} \Delta, \mathscr{S}) \rightarrow H_{18}\left(\mathbb{R} \mathscr{C}_{3}, \mathscr{S}\right)-$ гомоморфизм, индуцированный включением. Группа $H_{18}(\mathbb{R} \Delta, \mathscr{S})$ порождается фундаментальньми классами компонент связности множества $\Delta_{0}$, т.е. жесткими изотопическими классами кубик с единственной невырожденной двойной точкой. Такая кубика (в предположении, что особой точкой является (1:0:0:0)) задается уравнением

$$
x_{0} p_{2}\left(x_{1}, x_{2}, x_{3}\right)+p_{3}\left(x_{1}, x_{2}, x_{3}\right)=0
$$

где $p_{2}$ и $p_{3}$ - вешественные однородные многочлены степеней 2 и 3 соответственно. Точка (1: $0: 0: 0)$ - единственная особенность поверхности, причем она является невырожденной двойной точкой тогда и только тогда, когда квадрика $C_{2}=\left\{p_{2}=0\right\}$ неособа и кубика $C_{3}=\left\{p_{3}=0\right\}$ пересекает $C_{2}$ трансверсально. (Заметим, что $C_{3}$ может быть особой.) Через любые шесть различных точек кривой $C_{2}$ можно провести кубическую кривую, не содержащую $C_{2}$, причем такие кривые образуют трехмерное аффинное пространство. Поэтому $\Delta_{0}$ имеет не более пяти компонент связности: либо $\mathbb{R} C_{2}=\varnothing$, либо $\mathbb{R} C_{2} \cong S^{1}$ и кривые $C_{2}, C_{3}$ имеют $2 i, i=0,1,2,3$, вешественных точек пересечения. Возмущая особые поверхности, полученные таким образом, можно построить все пять топологических типов неособых кубических поверхностей.

Остается доказать, что

$$
\operatorname{dim} \operatorname{Kerin}_{*} \leqslant 4=\operatorname{dim} H_{18}(\mathbb{R} \Delta, \mathscr{S})-1 .
$$

Для этого опять в силу двойственности Пуанкаре достаточно построить топологическую окружность в $\mathbb{R} \mathscr{C}_{3} \backslash \mathscr{S}$, трансверсально пересекающую $\Delta_{0}$ в двух точках различных стратов. Такая окружность содержится в версальном двухпараметрическом возмушении каспидальной поверхности, задаваемой уравнением

$$
x_{0}\left(x_{1}^{2}+x_{2}^{2}\right)+x_{3}^{3}+x_{2}^{3}=0 .
$$

ЗАмечАниЕ. Компонента $V_{q}$ вещественной части неособой кубической поверхности расположена топологически в $\mathbb{R P} \mathbb{P}^{3}$ как стандартная $\mathbb{R} \mathbb{P}^{2}$ с ручками, приклеенными незаузленно и незацепленно. Это следует, например, из указанного вьше построения таких поверхностей.

3.5.3. Вещественные поверхности степени 4. Систематическое изучение топологии поверхностей степени 4 было предпринято в начале XX века K. Рооном и Д. Гильбертом. (Гильберт включил этот вопрос в свою 16-ю проблему.) Однако первые полные доказательства некоторых из их утверждений появились позже и принадлежали И.Г. Петровскому и О.А. Олейник (оценка числа сферических компонент) и Р. Тому (оценка тотального числа Бетти). Изучение было продолжено в 60-х и 70-х годах Г. А. Уткиньм, а классификация была завершена В. М. Харламовым [57], [60] (топологическая и изотопическая классификации; один изотопический класс был запрещен совместно с В.В. Никулиным) и В.В. Никулиным [88] (грубая проективная классификация). Киральность поверхностей степени 4 изучалась В. М. Харламовым [61], [62]. 
Как и у любой неособой вешественной поверхности четной степени, вешественная часть неособой квартики ориентируема. Ее расположение в $\mathbb{R} \mathbb{P}^{3}$ является довольно простым: она изотопна объединению эллипсоидов и гиперболоидов с незаузленными и незацепленными ручкми. За одним исключением компоненты лежат вне друг друга; в исключительном случае вешественная часть является гнездом из двух сфер. (В большинстве случаев эти результаты можно вывести из теоремы Безу.)

За исключением гнезда из двух сфер положение поверхности $\mathbb{R} X$ в $\mathbb{R} \mathbb{P}^{3}$ с точностью до изотопии определяется образами гомоморфизмов включения $H_{1}\left(C_{j}\right) \rightarrow H_{1}\left(\mathbb{R P}^{3}\right)=$ $\mathbb{Z}_{2}$, где $C_{j} \subset \mathbb{R} X-$ компоненты поверхности $\mathbb{R} X$. (Это следует из общих теорем трехмерной топологии и того, что ручки вешественной части незаузлены и незацеплены.) Для несферических компонент мы используем обозначение $S_{p}^{0}\left(\right.$ соответственно $\left.S_{p}^{1}\right)$, если образ указанного гомоморфизма тривиален (соответственно нетривиален). Далее, $n S$ обозначает $n$ сфер, лежащих вне друг друга, а $S(S)$ - гнездо из двух сфер.

Для поверхностей степени 4 все четыре класификации (топологическая, изотопическая, грубая проективная и жесткая) различны.

3.5.4. КЛАССИФИКАЦИИ ВЕШЕСТВЕННЫХ ПОВЕРХНОСТЕЙ СТЕПЕНИ 4. Вещественная неособая поверхность степени 4 с точностью до жесткой изотопии определяется изотопическим типом своей вещественной части $\mathbb{R} X$, комплексным типом $\left(\mathrm{I}_{0}, \mathrm{I}_{\mathrm{hp}}\right.$ или $\mathrm{II}$, см. 2.6) и тем, является ли поверхность киральной, т.е. жестко изотопной своему зеркальному образу, или нет. Изотопическими типами вещественных частей являются все типь, получающиеся последовательностью морсовских упрощений (см. 3.7.1) из следующих әкстремальных типов:

(1) $M$-поверхности: $S_{10}^{1} \sqcup S, S_{6}^{1} \sqcup 5 S, S_{2}^{1} \sqcup 9 S$;

(2) $(M-2)$-поверхности: $S_{7}^{1} \sqcup 2 S, S_{3}^{1} \sqcup 6 S, S_{a}^{0} \sqcup(9-a) S, 0 \leqslant a \leqslant 9$;

(3) пары торов: $S_{1}^{1} \sqcup S_{1}^{1}, S_{1}^{0} \sqcup S_{1}^{0}$;

(4) гнездо из двух сфер: $S(S)$.

Все некиральные поверхности образуют следующие изотопические типь: $p S \sqcup S_{q}^{0}$, $p \geqslant 3, q \geqslant 3$, и $p S \sqcup S_{q}^{1}, p \geqslant 4$. Все комплексные типь указаны в табл. 1 и 2.

ЗАмЕчАнИЕ. Из 3.5.4 следует, что за исключением случая, когда $\mathbb{R} X=S(S)$, изотопический тип вещественной части $\mathbb{R} X$ вешественной квартики в $\mathbb{P}^{3}$ определяется ее топологическим типом и тем, стягиваема ли $\mathbb{R} X$ в $\mathbb{R} \mathbb{P}^{3}$ или нет.

3.5.5. Поверхности степени 5. Для степеней $\geqslant 5$ имеет место следующее явление: с одной стороны, любая поверхность, полученная малой деформацией поверхности данной степени в $\mathbb{P}^{3}$, вкладьвается в $\mathbb{P}^{3}$ (как поверхность той же степени); с другой стороны, большая деформация может дать поверхность, которую нельзя вложить в $\mathbb{P}^{3}$. (Стоит отметить, что для степени 4 ситуация прямо противоположная: малой деформацией неособую квартику можно сделать непроективной и, с другой стороны, любую поверхность из того же деформационного класса, т.е. $K 3$-поверхность, см. 3.7.2 ниже, малой деформацией можно превратить в неособую квартику. Это объясняет то, почему топологическая классификация вешественных неособых поверхностей степени 4 совпадает с топологической классификацией вещественных $K 3$-поверхностей.) 

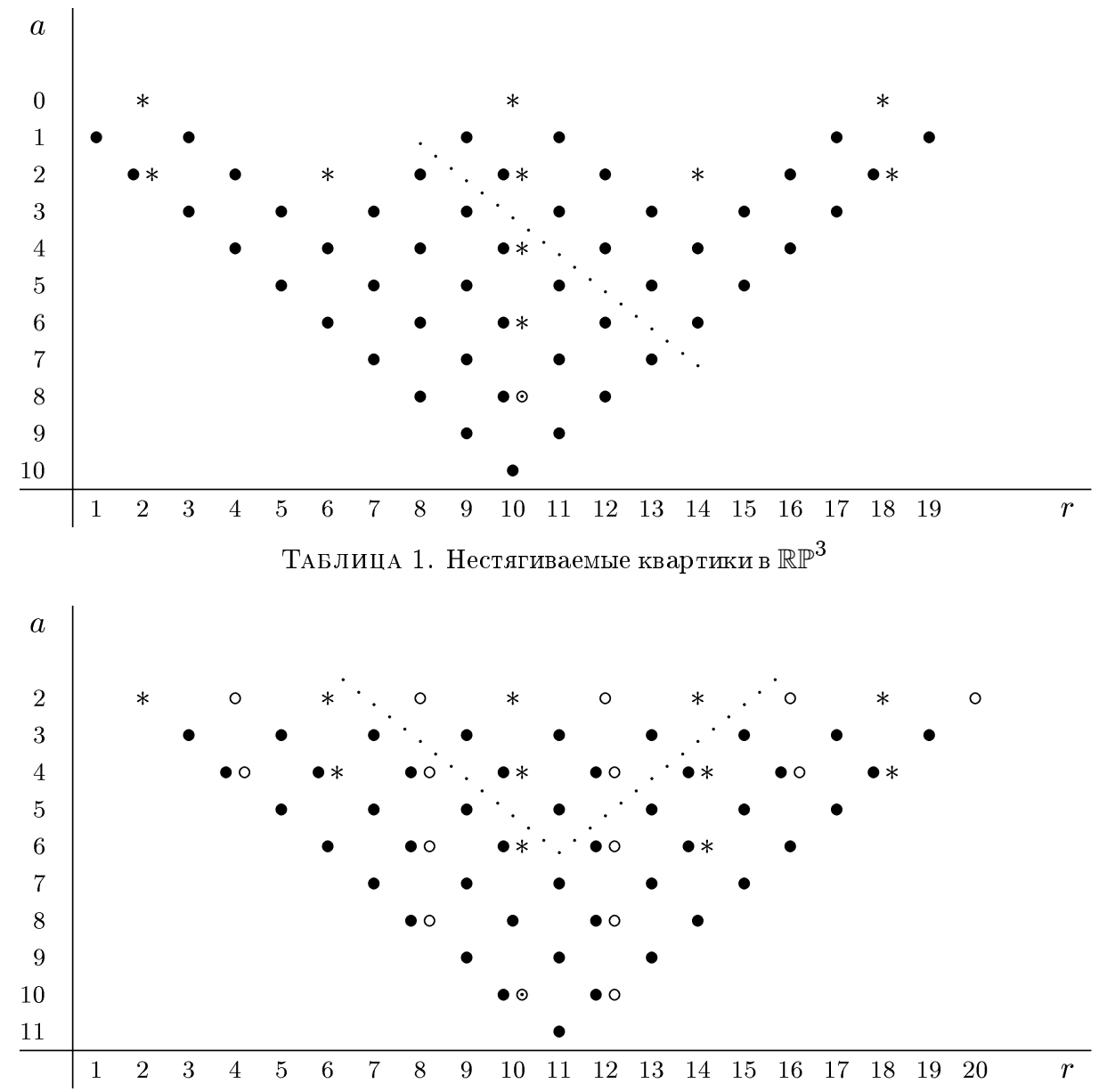

ТАБлица 2. Стягиваемые квартики в $\mathbb{R P}^{3}$

* и $\odot$ обозначают поверхность типа $\mathrm{I}_{0}$, ○ обозначает поверхность типа $\mathrm{I}_{\mathrm{hp}}$, а $\bullet$ обозначает поверхность типа II. Знаком $\odot$ отмечены поверхности типа $\mathrm{I}_{0}$ с $\mathbb{R} X=2 S_{1}((a, r)=(8,10)$ в табл. 1$)$ и $\mathbb{R} X=\varnothing((a, r)=(10,10)$ в табл. 2$)$; для поверхностей с $a+r=22$ (правый край табл. 2) $\mathbb{R} X=(r-10) S$; для остальных поверхностей $\mathbb{R} X=S_{g} \sqcup k S$, где $g=\frac{1}{2}(22-a-r)$ и $k=\frac{1}{2}(r-a)$. Поверхности ниже (соответственно выше) пунктирных линий являются (соответственно не являются) жестко изотопными своим зеркальным образам.

Для степени 5 указанное явление было изучено Е. Хорикавой [50]. Он доказал, что база версальной деформации неособой квинтики состоит из двух гладких неприводимых компонент $M_{0}, M_{1}$ с нормальньм пересечением, причем $\operatorname{dim}_{\mathbb{C}} M_{0}=\operatorname{dim}_{\mathbb{C}} M_{1}=40$ и $\operatorname{dim}_{\mathbb{C}} M_{0} \cap M_{1}=39$. Точки множества $M_{0} \backslash\left(M_{0} \cap M_{1}\right)$ соответствуют неособым квинтикам, точки множества $M_{1} \backslash\left(M_{0} \cap M_{1}\right)$ - двулистным накрытиям поверхности $\mathbb{P}^{1} \times \mathbb{P}^{1}$, а точки множества $M_{0} \cap M_{1}$ - двулистньп накрытиям квадратичного конуса $\Sigma_{2}$. Ясно, что деформацию вешественной квинтики можно сделать эквивариантной, а ее члены превратить в квинтики того же вещественного деформационного класса. Это наблюдение используется в той части доказательства предложений 3.5.6 и 3.5.7, где обосновывается сушествование поверхностей. 
3.5.6. ПРЕДЛОЖЕНИЕ. Экстремальные значения чисел Бетти неособых квинтик в $\mathbb{P}^{3}$ находятся в пределах $22 \leqslant \max \beta_{0} \leqslant 25, \max \beta_{1}=45$ или 47. Для поверхностей из того же деформачионного класса $23 \leqslant \max \beta_{0} \leqslant 25$ и $\max \beta_{1}=47$.

ДокАЗАТЕльство. Если $X-$ неособая квинтика, то $h^{1,1}(X)=45, \beta_{*}(X)=55$ и $\sigma(X)=-35$. Поэтому верхние гранишы, указанные в формулировке, получаются из неравенств Смита и Комессатти 3.1.1., 3.1.2 и экстремального сравнения 2.7.1. Квинтики с $\beta_{1}=45$ построены В. М. Харламовым [63], квинтики с $\beta_{0}=22-$ И. В. Итенбергом и В. М. Харламовьм [51], а $\mathbb{C}$-деформационно эквивалентные квинтике поверхность с $\beta_{0}=23$ и поверхность с $\beta_{1}=47-\Phi$. Бианом [11].

3.5.7. ПРЕДЛОЖЕНИЕ. Вещественная часть $M$-поверхности, $\mathbb{C}$-деформаиионно әквивалентной неособой квинтике, может иметь только 5, 9, 13, 17, 21 или 25 компонент связности. М-квинтики с 5, 9, 13, 17 и 21 компонентами существуют.

ДокАЗАтЕльство. Другие значения запрешены экстремальным сравнением 2.7.1 и неравенством Смита 3.1.1. $M$-поверхности с 5, 9, 13, 17 и 21 компонентами построены В. М. Харламовым [63] (построение в случае 17 и 21 компонент использует некоторые вспомогательные кривые, построенные О.Я. Виро).

$M$-поверхности, построенные в [63], имеют следующие топологические типы:

$$
20 S \sqcup V_{13}, \quad 16 S \sqcup V_{21}, \quad 12 S \sqcup V_{29}, \quad 8 S \sqcup V_{37}, \quad 4 S \sqcup V_{45} .
$$

Их близнецами, полученными сменой знака двулистного накрытия, являются

$$
4 S \sqcup V_{43}, \quad 8 S \sqcup V_{35}, \quad 12 S \sqcup V_{27}, \quad 11 S \sqcup S_{1} \sqcup V_{25}, \quad 16 S \sqcup V_{19} .
$$

Среди других пар поверхностей, построенных к настоящему времени, имеются пары $19 S \sqcup S_{2} \sqcup V_{7}, 4 S \sqcup V_{43}$ и $19 S \sqcup S_{1} \sqcup V_{7}, 4 S \sqcup V_{45}$. Из построения вспомогательных кривых следует, что поверхности, полученные морсовскими упрошениями всех вьшеуказанных топологических типов, реализуются вешественными квинтиками. Совокупность поверхностей, полученных таким способом, никогда систематически не изучалась.

Поверхность с 22 компонентами, построенная Харламовым и Итенбергом в [51], имеет тип $21 S \sqcup V_{7}$. Ее близнецом является $S \sqcup S_{2} \sqcup V_{41}$ с $\beta_{1}=45$.

Недавно Б. Шевалье (неопубликовано) нашел несколько дополнительных примеров. Его подход выглядит иначе (он опирается на метод склеивания Виро), но есть основание считать, что оба подхода близки или даже эквивалентны: подход, основанный на теореме Хорикавы, тоже опирается на метод Виро построения вспомогательных кривых, а подход Шевалье тоже использует двулистные накрытия квадрик. Шевалье получил следующие поверхности, у которых тотальное число Бетти или эйлерова характеристика близки к экстремальным:

$$
\begin{gathered}
18 S \sqcup S_{1} \sqcup V_{9}, \quad 17 S \sqcup 2 S_{1} \sqcup V_{3}, \quad 17 S \sqcup S_{2} \sqcup V_{5}, \quad \ldots, \quad 4 S \sqcup S_{8} \sqcup V_{23}, \\
3 S \sqcup S_{1} \sqcup V_{39}, \quad 3 S \sqcup S_{7} \sqcup V_{27}, \quad 2 S \sqcup S_{1} \sqcup V_{41}, \quad S \sqcup S_{18} \sqcup V_{3}, \quad S \sqcup S_{10} \sqcup V_{21} .
\end{gathered}
$$


Стоит отметить, что если $M$-квинтика с 25 компонентами действительно существует, то согласно известным неравенствам и сравнениям она должна относиться к одному из следуюших топологических типов:

$$
24 S \sqcup V_{5}, \quad 23 S \sqcup S_{1} \sqcup V_{3}, \quad 23 S \sqcup S_{2} \sqcup V_{1}, \quad 22 S \sqcup S_{1} \sqcup S_{1} \sqcup V_{1} .
$$

Два последних типа запрещены (четвертый - О. Виро, а третий - Харламовым и Итенбергом, ср. [64]). Проблема существования $M$-поверхностей степени 5 двух оставшихся топологических типов остается открытой.

3.6. Рациональные поверхности. В этом обзоре под вещественной рациональной поверхностью мы подразумеваем рациональную (над $\mathbb{C}$ ) поверхность, наделенную вешественной структурой. Стоит подчеркнуть, что такая поверхность не обязана быть рациональной над $\mathbb{R}$; приведенная ниже теорема 3.6 .1 содержит соответствуюшие примеры. Классификация вешественных рациональных поверхностей была получена Комессатти [18]-[20]. Следуя Комессатти, ограничимся минимальньлми $($ над $\mathbb{R})$ вещественными рациональными поверхностями. (Напомним, что неособая поверхность $X$ назьвается (относительно) минимальной, если любое голоморфное отображение $X \rightarrow X^{\prime}$ степени 1 является биголоморфным. Вешественная поверхность $(X$, conj) минимальна над $\mathbb{R}$, если указанное вьше условие выполняется для conj-эквивариантных отображений. Поверхность минимальна над $\mathbb{C}$ тогда и только тогда, когда она не содержит (-1)-кривых, и минимальна над $\mathbb{R}$ тогда и только тогда, когда она не содержит вешественных $(-1)$-кривых и пар дизъюнктных комплексно сопряженных (-1)-кривых.)

3.6.1. МИНИМАЛЬНЫЕ ВЕШЕСТВЕННЫЕ РАЦИОНАЛЬНЫЕ ПОВЕРХНОСТИ. НИж્ приведен полный перечень жсестких изотопических типов минимальньх над $\mathbb{R}$ вещественных рациональных поверхностей:

(1) вещественная проективная плоскость $\mathbb{P}^{2}: \mathbb{R} X=V_{1}$;

(2) вещественная квадрика $\mathbb{P}^{1} \times \mathbb{P}^{1}$ одного из четырех типов: поверхность c $\mathbb{R} X=S_{0}, \mathbb{R} X=S_{1}$ и две неэквивалентные поверхности с $\mathbb{R} X=\varnothing ;$

(3) линейчатые рациональные поверхности $\Sigma_{m}, m \geqslant 2$ : $m$ четно: $\mathbb{R} X=\varnothing$ или $S_{1}, \quad$ т нечетно: $\mathbb{R} X=V_{2} ;$

(4) пространства вещественных расслоений на коники над $\mathbb{P}^{1}$, все приводимые слои которых вещественны и состоят из пар комплексно сопряженных исключительных кривых: $\mathbb{R} X=m S$, где $2 m \geqslant 4$ есть число приводимых слоев;

(5) поверхности Дель Пецчо степени $d=K^{2}=1$ или 2:

$$
d=1: \quad \mathbb{R} X=V_{1} \sqcup 4 S, \quad d=2: \quad \mathbb{R} X=3 S \text { или } 4 S .
$$

( $\mathrm{C}$ комплексной точки зрения поверхность Дель Пещцо степени $d \leqslant 9$ получается из $\mathbb{P}^{2}$ раздутием $(9-d)$ точек, лежаших в общем положении, т.е. так, что никакие три из них не лежат на одной прямой и никакие шесть не лежат на одной конике.) Заметим, что поверхность Дель Пещо степени 2 с $\mathbb{R} X=3 S$ можно также представить в виде пространства расслоения на коники над $\mathbb{P}^{1}$ с шестью приводимыми слоями. Отметим также, что в указанном перечне только поверхности (1)-(3) минимальны над $\mathbb{C}$.

Теорема 3.6.1 связана с классификацией антибирациональных преобразований плоскости $\mathbb{P}^{2}$, опирающейся, в свою очередь, на изучение фундаментальных точек 
таких преобразований. Изложение доказательства Комессатти на современном языке можно найти в [15].

3.7. Абелевы, $K 3$-поверхности и поверхности Энриквеса. Следующей группой в классификации комплексных алгебраических поверхностей являются поверхности с нулевой размерностью Кодаиры. Их минимальные модели над $\mathbb{C}$ исчерпываются абелевыми поверхностями, гиперэллиптическими поверхностями, $K 3$-поверхностями и поверхностями Энриквеса, причем каждый тип, за исключением гиперэллиптических поверхностей, образует одно деформационное семейство. Так как минимальная модель комплексной поверхности, имеюшей размерность Кодаиры $\geqslant 0$, единственна (см., например, [47]), она должна быть вещественной, если исходная поверхность вешественна. Поэтому достаточно рассматривать поверхности, минимальные над $\mathbb{C}$; любую другую поверхность можно получить из минимальной (над $\mathbb{C}$ ) последовательностью раздутий вешественных точек или пар комплексно сопряженных точек.

Классификация гиперэллиптических поверхностей еше не закончена. Ниже мы ограничиваемся тремя остальными вьшшеуказанными типами.

Абелева поверхность $X$ (гомеоморфная четырехмерному тору $S^{1} \times S^{1} \times S^{1} \times S^{1}$ ) характеризуется условиями $b_{1}(X)=4$ и $K_{X}=0$. Классификация антиголоморфных инволюций на таких поверхностях была получена Комессатти [20] и может быть описана следующим образом.

3.7.1. ВЕЩЕСТВЕННЫЕ АБЕЛЕВЫ ПОВЕРХНОСТИ. С точностью до эквивариантной деформации антиголоморфная инволючия сопј на комплексном торе $X$ определяется множеством вещественных точек $\mathbb{R} X$, если $\mathbb{R} X=\varnothing$, дейст-

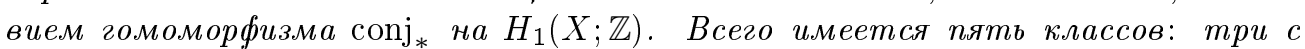
$\mathbb{R} X=k S_{1}, k=1,2,4$, и два $c \mathbb{R} X=\varnothing$.

Описание пространства модулей алгебраических абелевых поверхностей с непустой вешественной частью можно найти у Силёля [107].

НАБРосок докаЗАТЕЛьСтвА. Рассмотрим униформизацию $X=\mathbb{C}^{2} / L$, где $L \subset \mathbb{C}^{2}$ - решетка ранга 4, и поднимем conj до антиавтоморфизма $c$ пространства $\mathbb{C}^{2}$. Если $\mathbb{R} X \neq \varnothing$, это поднятие можно выбрать в виде инволюции, сохраняющей $L$. Поскольку $c$ имеет собственные числа \pm 1 , кратности которых равны 2 , сужение $\left.c\right|_{L}$ принадлежит одному из трех классов изоморфизма, и для каждого класса существует единственное с точностью до гомотопии эквивариантное вложение в пространство $\mathbb{C}^{2}$ (рассматриваемое со стандартным комплексным сопряжением).

Если $\mathbb{R} X=\varnothing$, те же аргументы применяются к присоединенному действию Ad conj на поверхности $X$ как на группе, а сама инволюция сопј есть композиция отображения Ad conj и аффинного сдвига на элемент $a \in \operatorname{Ker}(1+\operatorname{Ad}$ conj), которьй следует рассматривать по модулю $\operatorname{Im}(1-\mathrm{Ad}$ conj $)$. С точностью до изоморфизма имеются два случая: один с Ad conj $=\mathrm{id}$, другой с Ad conj $=\mathrm{id} \oplus \sigma$, где $\sigma$ - вешественная структура на такой эллиптической кривой $C^{\prime}=\mathbb{C} / L^{\prime}$, что $\mathbb{R} C^{\prime}=S^{1}$.

Комплексная $K 3$-поверхность $X$ характеризуется условиями $b_{1}(X)=0, K_{X}=0$. (Топологически поверхность $X$ - односвязное четырехмерное многообразие с $H_{2}(X ; \mathbb{Z})=2\left(-E_{8}\right) \oplus 3 U$.) Для описания классификации вешественных $K 3$-поверхностей введем следующее понятие: топологический тип (т.е. класс поверхностей 
с гомеоморфными вешественными частями) называется әкстремальным (в пределах фиксированного деформационного семейства комплексных поверхностей), если его нельзя получить из другого топологического типа морсовским упрощением, т.е. морсовской перестройкой, уменшаюшей тотальное число Бетти. (Морсовское упрощение есть удаление либо сферической компоненты, либо ручки.) Следующий результат принадлежит В. М. Харламову [57] и В.В. Никулину [88]:

3.7.2. ВЕШЕСТВЕННЫЕ $K 3$-ПОВЕРХНОСТИ. К3-поверхность $X$ с антиголоморфной инволюиией определяется, с точностью до әквивариантной деформации, топологическим типом своей вещественной части $\mathbb{R} X$ и типом вещественной структурь (т.е. тем, гомологично или не гомологично нулю $[\mathbb{R} X]$ в $H_{2}(X)$, см. 2.6). Топологическими типами вещественных частей являются те и только те типь, которые мохсно получить последовательностью морсовских упрощений из следующих экстремальных типов:

(1) $M$-поверхности: $S_{10} \sqcup S, S_{6} \sqcup 5 S, S_{2} \sqcup 9 S$;

(2) $(M-2)$-поверхности: $S_{7} \sqcup 2 S, S_{3} \sqcup 6 S$;

(3) пара торов: $2 S_{1}$.

Топологические типы вещественных частей вещественных К3-поверхностей перечислень в табл. 3 .

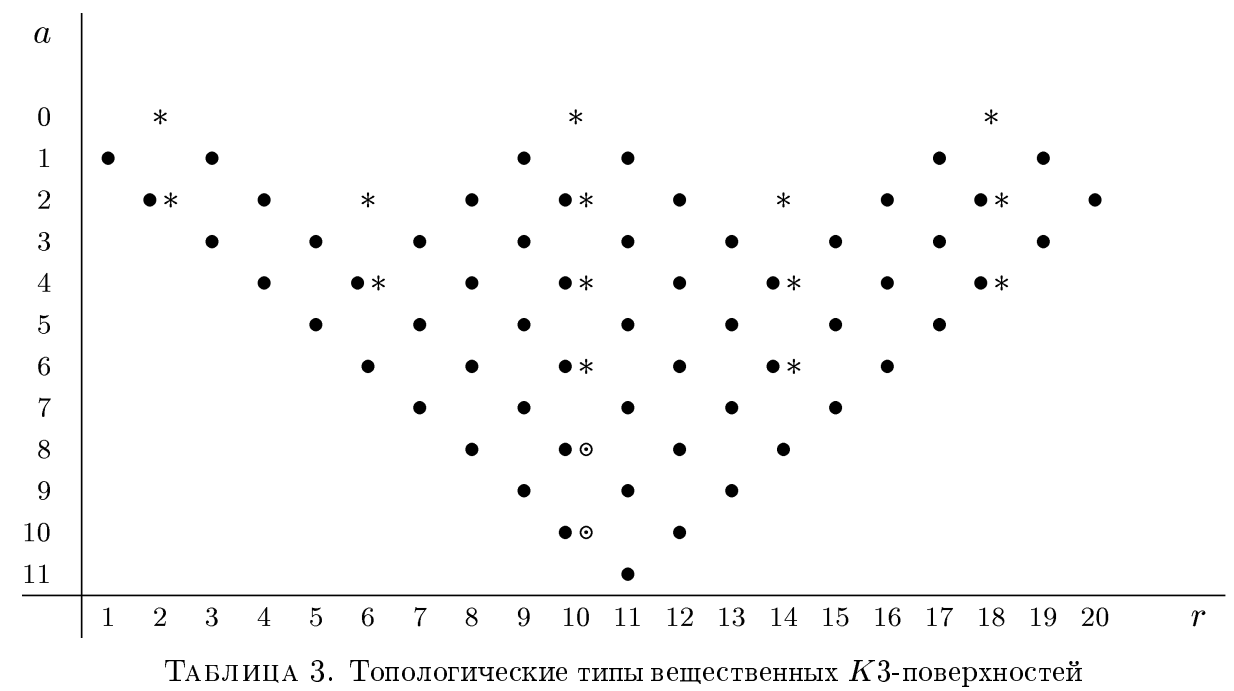

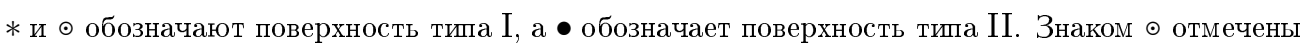
поверхности типа $\mathrm{I}$ с $\mathbb{R} X=2 S_{1}((a, r)=(8,10))$ и $\mathbb{R} X=\varnothing((a, r)=(10,10))$; для поверхностей с $a+r=22$ (правьй край таблицы) $\mathbb{R} X=(r-10) S$; для остальных поверхностей $\mathbb{R} X=S_{g} \sqcup k S$, где $g=\frac{1}{2}(22-a-r)$ и $k=\frac{1}{2}(r-a)$.

Доказательство теоремы 3.7.2 основано на глобальной теореме Торелли для $K 3$-поверхностей, см. [10], которая описьвает пространство модулей этих поверхностей, что сводит проблему классификации антиголоморфных инволюций на $K 3$-поверхностях с точностью до жестких изотопий к арифметической проблеме классификации инволютивных изометрий на $2\left(-E_{8}\right) \oplus 3 U$ с точностью до автоморфизма решетки. Мы опускаем детали (см. [88] или [29]). 
Поверхности оставшегося деформационного типа - поверхности Энриквеса - характеризуются условиями $2 K_{X}=0, K_{X} \neq 0$. (Другое определение состоит в следуюшем: $X$ - поверхность Энриквеса, если $\pi_{1}(X)=\mathbb{Z}_{2}$ и универсальное накрьвающее поверхности $X$ есть $K 3$-поверхность.) Как и в предыдущем случае, вешественная поверхность Энриквеса $X$ с точностью до деформации определяется топологией инволюции комплексного сопряжения conj: $X \rightarrow X$. Однако топология вешественной части $\mathbb{R} X$ и тип инволюции уже не являются достаточными для определения топологии инволюции; нужны более тонкие инварианты. Ниже мы приводим основные результаты деформационной классификации вешественных поверхностей Энриквеса; подробности и полный список деформационных классов можно найти в [29].

3.7.3. ТОПОЛОГИЯ ВЕЩЕСТВЕННОЙ чАСТИ. Имеются 87 mопологических muпов поверхностей Энриквеса с антиголоморфной инволюиией. Каждый из них мохсно получить последовательностью морсовских упрощений из одного из 22 әкстремальных типов, перечисленных ниже. Обратно, за исключением двух типов $6 S_{0}$ и $S_{1} \sqcup 5 S_{0}$ любой топологический тип, полученный таким способом, реализуется вещественной поверхностью Энриквеса.

Экстремальные типы исчерпываются следующим списком:

(1) $M$-поверхности:

\begin{tabular}{|c|c|c|c|}
\hline$\chi(\mathbb{R} X)=8:$ & $(\mathrm{b}$ & $\chi(\mathbb{R} X)=-8:$ & \\
\hline $4 V_{1} \sqcup 2 S_{0}$, & $2 V_{2} \sqcup 4 S_{0}$, & $V_{11} \sqcup V_{1}$ & $V_{8} \sqcup V_{4}$, \\
\hline$V_{2} \sqcup 2 V_{1} \sqcup 3 S_{0}$, & $V_{4} \sqcup 5 S_{0}$, & $V_{10} \sqcup V_{2}$, & $V_{7} \sqcup V_{5}$, \\
\hline$V_{3} \sqcup V_{1} \sqcup 4 S_{0}$, & $V_{2} \sqcup S_{1} \sqcup 4 S_{0}$, & $V_{9} \sqcup V_{3}$, & $\begin{array}{l}2 V_{6}, \\
V_{10} \sqcup S\end{array}$ \\
\hline
\end{tabular}

(2) $(M-2)$-поверхности с $\chi(\mathbb{R} X)=0$ :

$$
\begin{array}{llll}
V_{4} \sqcup 2 V_{1}, & V_{5} \sqcup V_{1} \sqcup S_{0}, & 2 V_{3} \sqcup S_{0}, & V_{6} \sqcup 2 S_{0}, \\
V_{3} \sqcup V_{2} \sqcup V_{1}, & V_{4} \sqcup V_{2} \sqcup S_{0}, & V_{4} \sqcup S_{1} \sqcup S_{0}, & 2 V_{2} \sqcup S_{1} ;
\end{array}
$$

(3) пара торов: $2 S_{1}$.

Пусть $X$ - вешественная поверхность Энривеса и $\widetilde{X} \rightarrow X$ - накрытие $K 3$-поверхностью. Вешественная структура conj на $X$ поднимается до двух коммутируюших вешественных структур $t^{(1)}, t^{(2)}: \widetilde{X} \rightarrow \widetilde{X}$, вешественные части $\mathbb{R} \widetilde{X}^{(1)}, \mathbb{R} \widetilde{X}^{(2)}$ которых не пересекаются. Образы $\mathbb{R} X^{(i)}$ поверхностей $\mathbb{R} \widetilde{X}^{(i)}, i=1,2$, также не пересекаются. Поэтому вешественная часть $\mathbb{R} X$ поверхности $X$ разбивается на два подмножества $\mathbb{R} X^{(1)}, \mathbb{R} X^{(2)}$ (являюшиеся объединениями целых компонент), которые называются половинами. Разбиение на половины $\mathbb{R} X=\mathbb{R} X^{(1)} \cup \mathbb{R} X^{(2)}$, назьваемое также фундаментальным разбиением, является важньм топологическим (и деформационным) инвариантом поверхности; оно записьвается в следующем виде

$$
\mathbb{R} X=\left\{\text { компоненты поверхности } \mathbb{R} X^{(1)}\right\} \sqcup\left\{\text { компоненты поверхности } \mathbb{R} X^{(2)}\right\} .
$$

Ясно, что можно говорить о типе $\left(\mathrm{I}_{0}, \mathrm{I}_{u}\right.$ или II) каждой половины $\mathbb{R} X^{(i)}$ отдельно, так же как и о типах (I или II) поверхностей $\mathbb{R} \widetilde{X}^{(i)}$. Более того, $\mathbb{R} X^{(1)}$ естественно отождествляется с множеством неподвижных точек инволюции, полученной из $t^{(1)}$ спуском 
в $\widetilde{X} / t^{(2)}$; поэтому можно также рассматривать тип поверхности $\mathbb{R} X^{(1)}$ в $\widetilde{X} / t^{(2)}$. Последний фактор диффеоморфен рациональной поверхности, если $\mathbb{R} X^{(2)} \neq \varnothing$, и поверхности Энриквеса в противном случае. В первом случае возможными типами являются I и II, в последнем - $\mathrm{I}_{0}, \mathrm{I}_{u}$ и II.

3.7.4. ПРЕДЛОЖЕнИЕ. Накрытие $\mathbb{R} \widetilde{X}^{(1)} \cup \mathbb{R} \widetilde{X}^{(2)} \rightarrow \mathbb{R} X$ является ориентирующим двулистныц накрытием.

3.7.5. ФУНДАМЕНТАЛЬНОЕ РАЗБИЕНИЕ. Каждая половина вещественной поверхности Энриквеса есть либо

(1) $\alpha V_{g} \sqcup a V_{1} \sqcup b S$ c $g>1, a \geqslant 0, b \geqslant 0, \alpha=0,1$, либо

(2) $2 V_{2}$, либо

(3) $S_{1}$.

За исключением случаев $\mathbb{R} X=k S$ и $\mathbb{R} X=V_{2 r} \sqcup k S$ фундаментальное разбиение вещественной части $\mathbb{R} X$ из перечня в 3.7 .3 реализуемо тогда и только тогда, когда оно удовлетворяет указанныцм условиям (1)-(3). Для исключительных случаев реализуемые разложсения перечислены на рис. 1.

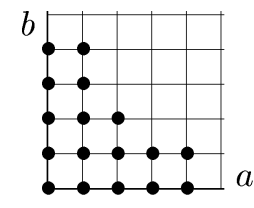

$\left\{a S_{0}\right\} \sqcup\left\{b S_{0}\right\}$, $\left\{V_{2} \sqcup a S_{0}\right\} \sqcup\left\{b S_{0}\right\}$

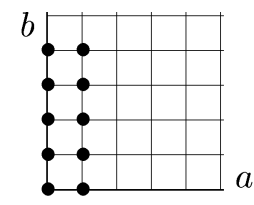

$\left\{V_{4} \sqcup a S_{0}\right\} \sqcup\left\{b S_{0}\right\}$

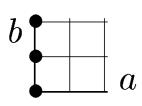

$\left\{V_{6} \sqcup a S_{0}\right\} \sqcup\left\{b S_{0}\right\}$

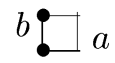

$\left\{V_{8} \sqcup a S_{0}\right\} \sqcup\left\{b S_{0}\right\}$, $\left\{V_{10} \sqcup a S_{0}\right\} \sqcup\left\{b S_{0}\right\}$

Рис. 1. Фундаментальные разложения исключительных вещественных поверхностей Энриквеса

Предложение 3.7.4, по сушеству, следует из того, что многообразие $\widetilde{X}$ спинорное, а $X$ - нет; поэтому инволюция Энриквеса обрашает спинорную ориентацию поверхности $\mathbb{R} \widetilde{X}$ (см. 2.9.1). Вместе с ограничениями на топологию поверхности $\mathbb{R} \widetilde{X}$ (см. 3.7.2) это дает возможные формы (1)-(3) половин поверхности $\mathbb{R} X$. При изучении исключительных случаев используются некоторые сравнения, аналогичные экстремальным сравнениям 2.7.1.

Для запрета почти всех несуществующих топологических типов достаточно соединить 3.7.4 (и 3.7.2) с неравенством Смита 3.1.1, обобщенным неравенством Комессатти-Петровского 3.1.2 и экстремальными сравнениями 2.7.1. После этого останется запретить только два типа: $S_{1} \sqcup 5 S_{0}, 3 V_{2}$ и одну серию: $S_{1} \sqcup V_{1} \sqcup \cdots$. Это делается с помощью спектральной последовательности Калинина (см. детали в [26]).

По техническим причинам вешественные поверхности Энриквеса разделяются на три класса: поверхности гиперболического, параболического и эллиптического типов - в зависимости от того, отрицательным, нулевым или положительным является минимум эйлеровой характеристики компонент поверхности $\mathbb{R} X$.

3.7.6. ДЕФОРМАцИОНнЫЕ КЛАССЫ. За исключением нескольких случаев, указанных ниже, деформационный класс вещественной поверхности Энриквеса $X$ 
с отмеченной половиной $\mathbb{R} X^{(1)}$ определяется топологией своего фундаментального разложсения. Исключениями являются:

(1) М-поверхности параболического и эллиптического типов, т.е. поверхности $с \mathbb{R} X=2 V_{2} \sqcup 4 S, V_{2} \sqcup 2 V_{1} \sqcup 3 S$ или $4 V_{1} \sqcup 2 S ;$ инвариантом является форма Понтрягина-Виро;

(2) поверхности с $\mathbb{R} X=2 V_{1} \sqcup 4 S$;

(3) другие поверхности с половиной $\mathbb{R} X^{(1)}=4 S$; дополнительнымии инвариантами являются типь $\mathrm{I}_{u}, \mathrm{I}_{0}$ или II половинь $\mathbb{R} X^{(1)}$ в $X$ и в $\widetilde{X} / t^{(2)}$;

(4) поверхности с $\mathbb{R} X=\left\{V_{10}\right\} \sqcup\{\varnothing\},\left\{V_{4} \sqcup S\right\} \sqcup\{\varnothing\},\left\{V_{2} \sqcup 4 S\right\} \sqcup\{\varnothing\} u$ $\{2 S\} \sqcup\{2 S\}$; дополнительным инвариантом является тип, $\mathrm{I}_{u}$ или $\mathrm{I}_{0}$, поверхности $\mathbb{R} X$ в $X$;

(5) поверхности $с \mathbb{R} X=2 V_{1} \sqcup 3 S$; дополнительный инвариант - тип, $\mathrm{I}_{u}$ или II, поверхности $\mathbb{R} X$ в $X$;

(6) поверхности с $\mathbb{R} X=\left\{S_{1}\right\} \sqcup\left\{S_{1}\right\}$.

Дополнительный инвариант, необходимый для различения деформационных классов в случаях 3.7.6(2) и (6), является более тонким и зависит от специальных арифметических свойств индуцированного $\left(\mathbb{Z}_{2} \times \mathbb{Z}_{2}\right)$-действия на $H_{2}(\widetilde{X} ; \mathbb{Z})$ (в случае $(2)$ ) или от топологии взаимного расположения двух половин $\mathbb{R} \widetilde{X}^{(i)}=S_{1} \sqcup S_{1}$ в $\widetilde{X}$ (в случае $(6))$.

Деформационная классификация вешественных поверхностей Энриквеса основана на двух различных подходах: трюке Дональдсона и арифметических вычислениях, использующих глобальную теорему Торелли для $K 3$-поверхностей. (Хотя каждый подход в отдельности мог бы решить проблему, сложность возникающих промежуточных задач сушественно зависит от рассматриваемой поверхности. Первый, более геометричный подход, хорошо работает для поверхностей гиперболического и параболического типов; второй - для поверхностей эллиптического типа.) Трюк Дональдсона использует гиперкэлерову структуру на $\widetilde{X}$ : можно изменить имеющуюся комплексную структуру так, чтобы инволюция Энриквеса стала антиголоморфной, а $t^{(2)}$ - голоморфной; тогда фактор $\widetilde{X} / t^{(2)}$ станет вещественной рациональной поверхностью (если $\left.\mathbb{R} X^{(2)} \neq \varnothing\right)$ и проблема сводится к изучению вешественных рациональных поверхностей с неособой анти-биканонической кривой (образом множества $\mathbb{R} X^{(2)}$ ). Второй подход использует глобальную теорему Торелли для установления взаимно однозначного соответствия между деформационными классами вешественных поверхностей Энриквеса и классами изоморфизма $\left(\mathbb{Z}_{2} \times \mathbb{Z}_{2}\right)$-действия (индуцированного инволюциями $t^{(1)}$ и $\left.t^{(2)}\right)$ на решетке с формой пересечений $H_{2}(\widetilde{X} ; \mathbb{Z})=2\left(-E_{8}\right) \oplus 3 U$; такое действие должно удовлетворять некоторым арифметическим условиям, чтобы обеспечить реализуемость антиголоморфными инволюциями и свободность действия композиции $t^{(1)} \circ t^{(2)}$. Возникающая арифметическая проблема решается с использованием теории дискриминантных форм, а связь между свойствами этого действия и топологией поверхности ( $X$, conj) устанавливается, в основном, с помощью спектральной последовательности Калинина (см. детали в [29]).

\section{4. Кривые на поверхностях}

4.1. Сравнения. Топологическая кривая (т.е. дизъюнктное объединение вложенных окружностей) $L$ на топологической поверхности $S$ называется тотально го- 
мологичной нулю, если гомоморфизм включения $H_{1}(L) \rightarrow H_{1}(S)$ тривиален. Определим род топологической поверхности $S$ равенством

$$
g(S)=b_{0}(S)-\frac{1}{2}\left(\chi(S)+b_{0}(\partial S)\right) .
$$

(Как нам представляется, обшепринятое определение рода неориентируемой или несвязной поверхности отсутствует, поэтому мы выбрали наиболее удобное для наших целей. Безусловно, для связной ориентируемой поверхности наше определение дает обьчный род. В общем случае род есть число всех ручек, причем пленка считается как половина ручки.) Заметим, что поверхность рода 0 состоит из сфер с дырами, а поверхность рода $1 / 2$ состоит из сфиер с дырами и одной вещественной плоскости с дырами. Легко также проверить, что если замкнутая поверхность $S$ представлена в виде объединения $S_{1} \cup S_{2}$ двух поверхностей, обший край которых $\partial S_{1}=\partial S_{2}$ тотально гомологичен нулю в $S$, то $\operatorname{dim} \operatorname{Im}\left[\mathrm{in}_{*}: H_{1}\left(S_{i}\right) \rightarrow H_{1}(S)\right]=2 g\left(S_{i}\right), i=1,2$.

4.1.1. ТЕОРЕМА. Пусть $X$ - комплексная поверхность с вещественной структурой, $A$ - обильный четный вещественный дивизор на $X$ c $\mathbb{R} A \neq \varnothing u Y-$ двулистное накрывающее поверхности $X$ с ветвлением над А. Предположим, что $H_{1}(X)=0$. Следующие условия необходимы для того, чтобы многообразие $\mathbb{R} Y_{-}$бьло $M$ - или $(M-1)$-поверхностью: $X$ есть $M$-поверхность, кривая $\mathbb{R} A$ тотально гомологична нулю в $\mathbb{R} X$ и челиком лежит в одной компоненте поверхности $\mathbb{R} X$, а все другие компоненты этой поверхности принадлежат $\mathbb{R} X_{-}$ и тривиально накрываются поверхностью $\mathbb{R} Y_{-}$. Если әти условия выполнены, mo

(1) $\mathbb{R} Y_{-}$есть $M$-поверхность тогда и только тогда, когда $A$ есть $M$-кривая $u \mathbb{R} X_{+}$- поверхность рода $0 ;$ в этом случае $\chi\left(\mathbb{R} X_{+}\right)=\frac{1}{4}[A]^{2} \bmod 8 ;$

(2) $\mathbb{R} Y_{-}$есть $(M-1)$-поверхность тогда и только тогда, когда либо А есть $(M-1)$-кривая $и \mathbb{R} X_{+}-$поверхность рода 0 , либо $A$ есть $M$-кривая $u$ $\mathbb{R} X_{+}-$поверхность рода $1 / 2 ;$ в этом случае $\chi\left(\mathbb{R} X_{+}\right)=\frac{1}{4}[A]^{2} \pm 1 \bmod 8$.

ДокаЗАтельство. Пусть $X, Y$ и $A$ являются соответственно $\left(M-d_{X}\right)$-поверхностью, $\left(M-d_{Y}\right)$-поверхностью и $\left(M-d_{A}\right)$-кривой. Используя точные последовательности пар $(\mathbb{R} X, \mathbb{R} A)$ и $\left(\mathbb{R} X, \mathbb{R} X_{+}\right)$, очевидное равенство $\beta_{*}\left(\mathbb{R} X, \mathbb{R} X_{+}\right)=\beta_{*}\left(\mathbb{R} X_{-}\right)$ и выражения для $\beta_{*}\left(\mathbb{R} Y_{-}\right)$и $\beta_{*}(Y)$, находяшиеся соответственно в левой и правой частях последнего неравенства в 3.1 .1 (ср. 2.3.2 и его доказательство), получаем, что

$$
d_{Y}=2 d_{X}+d_{A}+2\left(\beta_{0}(\mathbb{R} X)-c_{-}-1\right)+\left(\delta+\varepsilon_{+}\right)-2 \beta_{1}(X),
$$

где $c_{-}-$число замкнутых компонент поверхности $\mathbb{R} X_{-}$, накрытых тривиально, и

$$
\begin{aligned}
\delta & =\operatorname{dim} \operatorname{Im}\left[\operatorname{in}_{*}: H_{1}(\mathbb{R} A) \rightarrow H_{1}(\mathbb{R} X)\right], \\
\varepsilon_{+} & =\operatorname{dim} \operatorname{Im}\left[\operatorname{in}_{*}: H_{1}\left(\mathbb{R} X_{+}\right) \rightarrow H_{1}(\mathbb{R} X)\right] .
\end{aligned}
$$

Отсюда следуют условия того, что $\mathbb{R} Y$ - есть $M$ - или $(M-1)$-поверхность. ${ }^{3}$ Сравнения получаются из основных экстремальных сравнений 2.7 .1 , примененных к $\mathbb{R} Y_{-}$ и $\mathbb{R} X$.

\footnotetext{
${ }^{3}$ В условиях теоремы формула (4.1.2), как нетрудно проверить, равносильна формуле $d_{Y}=$ $2 d_{X}+d_{A}+2\left(\beta_{0}(\mathbb{R} X)-c_{-}-1\right)+2 \delta+2 g\left(\mathbb{R} X_{+}\right)$, из правой части которой надо вычесть $2 \beta_{1}(X)$, если отбросить условие $H_{1}(X)=0$. Последняя формула (и указанное ее обобщение) доказываются так же, как и (4.1.2), но при этом нет необходимости вводить $\varepsilon_{+} \cdot-$ Примеч. перев.
} 
Заметим, что, в отличие от случая плоских кривых, в общем случае $\mathbb{R} Y_{-}$и $\mathbb{R} Y_{+}$ не могут быть одновременно близки к $M$-поверхностям. Точнее, рассуждая как в доказательстве теоремы 4.1.1, получаем, что (в очевидных обозначениях) $d_{Y_{-}}+d_{Y_{+}}-$ $\beta_{2}(X)=\beta_{2}(X)+\beta_{*}(A)-\left(\beta_{*}\left(\mathbb{R} X_{-}\right)+\beta_{*}\left(\mathbb{R} X_{+}\right)-2 c_{0}\left(\mathbb{R} X_{-}\right)-2 c_{0}\left(\mathbb{R} X_{+}\right)\right)$, и из 3.1.1 заключаем, что

$$
d_{Y_{-}}+d_{Y_{+}} \geqslant \beta_{2}(X) .
$$

Выясним, когда для указанных вьше $X, A$ и $Y$ накрытие $\mathbb{R} Y_{-} \rightarrow \mathbb{R} X$ - тривиально и когда $\mathbb{R} Y_{-}$ориентируемо. Поскольку $A$ - четньй и обильньй дивизор, существует такая вешественная кривая $E \subset X$, что $2[E]$ есть нечетное кратное класса $[A]$. (Действительно, можно разделить $[A]$ на 2 и заменить полученньй дивизор достаточно большим нечетным кратным так, чтобы он стал эффективньм.) Обозначим через $e$ элемент группы ${ }^{\infty} E_{2}(X)$, см. А.2, определяемый классом $[E]$. Так как $H_{2}(X ; \mathbb{Z})$ свободно от кручения и $\mathrm{bv}_{2}[\mathbb{R} E]=e$, класс $e$ и образ класса $[\mathbb{R} E]$ в $H_{1}(\mathbb{R} X)$ однозначно определены классом $[A]$. Следуюшее утверждение сразу следует из определения характеристического класса двулистного накрытия и мультипликативности гомоморфизма bv 2 , см. А.2.2.

4.1.3. Для указанных выше $X, A$ и Е характеристический класс накрытия $\mathbb{R} Y_{-} \rightarrow \mathbb{R} X_{-}$, рассматриваемый как гомоморфизм $H_{1}\left(\mathbb{R} X_{-}\right) \rightarrow \mathbb{Z}_{2}$, есть сужение на $H_{1}\left(\mathbb{R} X_{-}\right)$отображсения $x \mapsto \mathrm{bv}_{2} x \circ e=x \circ[\mathbb{R} E]$.

4.1.4. СлеДСтвИЕ. Для указанных выще $X, A$ и $E$, если $\mathbb{R} X_{+}-$поверхность рода 0 (или рода $1 / 2)$, проекиия $\mathbb{R} Y_{-} \rightarrow \mathbb{R} X_{-}$есть ориентирующее двулистное накритие тогда и только тогда, когда $[\mathbb{R} E]=w_{1}(\mathbb{R} X)($ соответственно $[\mathbb{R} E]=$ $w_{1}(\mathbb{R} X)+\varkappa c$, әде с есть класс односторонней окружности в $\mathbb{R} X_{+}$и $\left.\varkappa \in \mathbb{Z}_{2}\right)$.

Если $X$ есть $M$-поверхность, $[\mathbb{R} E]=w_{1}(\mathbb{R} X)$ тогда и только тогда, когда $\frac{1}{2}[A]$ есть характеристический әлемент в $H_{2}(X)$.

Заметим, что $\frac{1}{2}[A]$ - характеристический элемент в $H_{2}(X)$ тогда и только тогда, когда $Y$ спинорное. Это следует из формулы проекции для $w_{2}$ и того, что проекция накрытия индуцирует эпиморфизм $H_{2}(Y) \rightarrow H_{2}(X)$, см. 2.2.2. Соединяя 4.1.1 и 4.1.4 со сравнением Никулина 3.1 .5 , получаем следуюший результат.

4.1.5. СРАвнЕНИЕ НИкУлИНА Для КРИвЫХ. Пусть $Х-$ комплексная поверхность с вещественной структурой, $H_{1}(X)=0$ и $A$ - обильный четный дивизор на Х. Предположим, что

- Х есть $M$-поверхность и А есть $М$-кривая,

- кривая $\mathbb{R} A$ не пустая и челиком лежит в одной компоненте поверхнос$m u \mathbb{R} X$,

- другие компоненты поверхности $\mathbb{R} X$ принадлежат $\mathbb{R} X$ - и ориентируемьи,

- $\mathbb{R} A$ тотально гомологична нулю в $\mathbb{R} X$,

- $\frac{1}{2}[A]-$ характеристический әлемент в $H_{2}(X)$.

Предположим далее, что для некоторого челого $r \geqslant 0$ әйлеровы характеристики всех замкнутых компонент поверхности $\mathbb{R} X_{-}$делятся на $2^{r+1}$, а эйлеровы характеристики остальных компонент делятся на $2^{r}$ для некоторого целого $r \geqslant 0$. Тогда:

- если $\mathbb{R} X_{+}-$поверхность рода 0, то $\chi\left(\mathbb{R} X_{-}\right)=0 \bmod 2^{r+3} ;$

- если $\mathbb{R} X_{+}-$поверхность рода $1 / 2$, то $r=0$ и $\chi\left(\mathbb{R} X_{-}\right)= \pm 1 \bmod 8$. 
ЗАмЕчАниЕ. Дополнительное сравнение Фидлера, см. [33], полученное с помощью формулы Рохлина-Гийю-Марена для плоских кривых, также можно распространить на случай кривых на поверхностях.

4.2. Обобщения формулы Рохлина для комплексных ориентаций. Рассмотрим неособую комплексную поверхность $X$ с вещественной структурой и разбиваюшую вешественную кривую $A \subset X$ без вешественных особенностей. Через $A^{k}$ обозначим $\mathbb{R}$-компоненту кривой $A$ и предположим, что каждая компонента $A^{k}$ неособа над $\mathbb{C}$. Пусть $C_{1}, \ldots, C_{n}-$ компоненты связности поверхности $\mathbb{R} X$, и для каждого $i=1, \ldots, n$ пусть $C_{i j}, j=1, \ldots, m_{i}$, - ориентируемые компоненты множества $C_{i} \backslash \mathbb{R} A$. Если $C_{i}$ ориентируема, зафиксируем ее ориентацию и наделим $C_{i j}$ индуцированной ориентацией; в противном случае наделим $C_{i j}$ произвольной ориентацией. Обозначим через $\left[C_{i}\right]$ (для ориентируемой $C_{i}$ ) и $\left[C_{i j}\right]$ фундаментальные классы в $H_{2}(\mathbb{R} X, \mathbb{R} A ; \mathbb{Z}) \subset H_{2}(\mathbb{R} X, \mathbb{R} A ; \mathbb{Q})$, соответствуюшие выбранньм ориентациям. Ясно, что $\left[C_{i j}\right]$ являются образуюшими группы $H_{2}(\mathbb{R} X, \mathbb{R} A ; \mathbb{Z})$ и что $\left[C_{i}\right]=\sum_{j=1}^{m_{i}}\left[C_{i j}\right]$, если $C_{i}$ ориентируема.

Вместе с кривой $A$ разбивающей является и любая ее $\mathbb{R}$-компонента, и потому $\mathbb{R} A^{k}$ разделяет $A^{k}$ на две половины $A_{ \pm}^{k}$. Пусть $\left[A_{ \pm}^{k}\right] \in H_{2}(X, \mathbb{R} A ; \mathbb{Z})$ - класс, реализуемый стандартно ориентированной половиной $A_{ \pm}^{k}$. Ясно, что $\left[A_{+}^{k}\right]+\left[A_{-}^{k}\right]=\left[A^{k}\right]$. (Мы отождествляем $\left[A^{k}\right],\left[A_{ \pm}^{k}\right]$ и т. д. с их образами при различных гомоморфизмах включения и коэффициентных гомоморфизмах.) Половины $A_{ \pm}^{k}$ индуцируют пару противоположных ориентаций на $\mathbb{R} A^{k}$, называемых комплексными ориентациями. Соответствуюшие фундаментальные классы обозначаются через $\left[\mathbb{R} A_{ \pm}^{k}\right]$. Положим $\left[\mathbb{R} A_{ \pm}\right]=$ $\sum\left[\mathbb{R} A_{ \pm}^{k}\right]$.

Пусть in: $A \rightarrow X$ - включение. Простейшее обобщение формулы Рохлина 1.1 (указанное В. И. Звониловым [129] при немного более сильных ограничениях) получается в предположении, что $\left[\mathbb{R} A_{+}\right]=0$ в $H_{1}(\mathbb{R} X ; \mathbb{Q})$. Это предположение равносильно сушествованию рациональных чисел $x_{i j}, i=1, \ldots, n, j=1, \ldots, m_{i}$, для которых

$$
\left[\mathbb{R} A_{+}\right]=\partial \sum x_{i j}\left[C_{i j}\right]
$$

Если $C_{i}$ неориентируема, $x_{i j}$ однозначно определяются кривой $\mathbb{R} A \subset \mathbb{R} X$ и ее комплексной ориентацией. Если $C_{i}$ ориентируема, класс $\sum_{j=1}^{m_{i}} x_{i j}\left[C_{i j}\right]$ определен с точностью до класса, кратного $\left[C_{i}\right]$. В этом случае, если $C_{i}$ не тор, числа $x_{i j}$ однозначно определяются условием $\sum x_{i j} \chi\left(C_{i j}\right)=0$. (Если $C_{i}-$ тор, число $\sum x_{i j} \chi\left(C_{i j}\right)$ однозначно определено кривой $\mathbb{R} A$ и ее комплексной ориентацией.)

Обозначим через $x$ образ класса $\sum x_{i j}\left[C_{i j}\right]$ в $H_{2}(X, \mathbb{R} A ; \mathbb{Q})$. Существуют единственные классы $\xi_{+}, \xi_{-} \in H_{2}(X ; \mathbb{Q})$, переходящие соответственно в $\left[A_{+}\right]-x,\left[A_{-}\right]+x$ при гомоморфизме релятивизации. Ясно, что $\xi_{+}+\xi_{-}=[A]$. Заметим, что, в отличие от случая плоских кривых, эти классы могут не определяться комплексной ориентацией кривой $\mathbb{R} A$; поэтому они являются инвариантом жесткой изотопии разбивающей кривой в $X$. Согласно Звонилову сушествуют примеры, когда этот инвариант различает кривые с одинаковыми комплексными ориентациями.

Если $A$ неособа, то $A_{+} \cap A_{-}=\mathbb{R} A$. В обшем случае $A_{+} \cap A_{-}$отличается от $\mathbb{R} A$ конечным числом точек. Обозначим через $d^{+-}$число этих точек с учетом их кратностей. Ясно, что $0 \leqslant d^{+-} \leqslant \frac{1}{2} \sum_{i<j}\left[A_{i}\right] \circ\left[A_{j}\right]$. 
4.2.2. ТЕОРеМА. При сделанньх предположсениях относительно $A$ и $X$ пусть, кроме того, in $_{*}\left[\mathbb{R} A_{+}\right]=0$ в $H_{1}(\mathbb{R} X ; \mathbb{Q})$. Тогда

$$
\xi_{+} \circ \xi_{-}=d^{+-}+\sum_{i, j=1}^{n, m_{i}} x_{i j}^{2} \chi\left(C_{i j}\right) .
$$

Более того, для каждой ориентируемой компоненты $C_{i}$ поверхности $\mathbb{R} X$

$$
\xi_{+} \circ\left[C_{i}\right]=-\xi_{-} \circ\left[C_{i}\right]=\sum_{j=1}^{m_{i}} x_{i j} \chi\left(C_{i j}\right) .
$$

ДокАЗАТЕЛЬСтво. Эти утверждения доказываются аналогично формуле Рохлина с использованием лагранжева свойства поверхности $\mathbb{R} X$ (т.е. того, что умножение на $\sqrt{-1}$ переводит касательное расслоение подмногообразия $\mathbb{R} X$ в его нормальное расслоение с противоположной ориентацией).

\subsection{3. ДополнениЕ. Справедливы равенства $\xi_{+} \circ[A]=\xi_{-} \circ[A]=\frac{1}{2}[A]^{2}$.}

В случае проективной плоскости $\xi_{+}=\xi_{-}=\frac{1}{2}[A]$, и замена левой части первого равенства в 4.2 .2 на $\frac{1}{4}[A]^{2}$ дает формулу Рохлина 1.1 (а также формулу Рохлина-Мишачева для кривых нечетной степени и формулу Рохлина-Звонилова-Виро для приводимых кривых). В обшем случае нельзя считать, что $\xi_{+}=\xi_{-}$, и придать желаемьй вид вьшеуказанной замене формулы Рохлина. Сравнивая точные последовательности пар $(A, \mathbb{R} A)$ и $(X, \mathbb{R} X)$, получаем, что равенства in $*\left[\mathbb{R} A_{+}\right]=0$ и $\xi_{+}=\xi_{-}$ вьполнены (при подходящем выборе чисел $x_{i j}$ ) тогда и только тогда, когда ядро гомоморфизма включения $H_{2}(A, \mathbb{R} A ; \mathbb{Q}) \rightarrow H_{2}(X, \mathbb{R} X ; \mathbb{Q})$ нетривиально. Имеется другое достаточное условие для $\xi_{+}=\xi_{-}$, которое следует из второго равенства в 4.2 .2 и того очевидного факта, что класс $\xi_{+}-\xi_{-}$инвариантен относительно conj $_{*}$ :

4.2.4. ПРЕДЛОЖЕНИЕ. Предположим, ито $\operatorname{in}_{*}\left[\mathbb{R} A_{+}\right]=0$ в $H_{1}(\mathbb{R} X ; \mathbb{Q})$ и что подпространство $H^{+}$инвариантных относительно $\operatorname{conj}_{*}$ классов в $H_{2}(X ; \mathbb{Q})$ порождается фундаментальными классами ориентируемых компонент поверхности $\mathbb{R} X$. Тогда $\xi_{+}=\xi_{-}$, если коэффичиенты $x_{i j}$ выбраны так, что $\sum_{j=1}^{m_{j}} x_{i j} \chi\left(C_{i j}\right)=0$ для каждой ориентируемой компоненты $C_{i}$ поверхности $\mathbb{R} X$. (При этих условиях последнее равенство автоматически выполнено для всех компонент, гомеоморфных тору.)

Если $X$ кэлерово, в силу равенства $\operatorname{dim} H^{+}=\frac{1}{2}\left(b_{2}(X)+\chi(\mathbb{R} X)\right)-1$ последнее условие в 4.2.4 выполнено, например, в следуюших случаях:

(1) $b_{2}(X)+\chi(\mathbb{R} X)=2$;

(2) $\mathbb{R} X$ - ориентируемая $M$-поверхность, $b_{0}(\mathbb{R} X) \leqslant 2$ и $\mathbb{R} X$ не пара торов;

(3) $\mathbb{R} X$ - ориентируемая $(M-1)$-поверхность, $b_{0}(\mathbb{R} X)=1$ и $\mathbb{R} X$ не тор.

ЗАМЕЧАНИЕ: ВАРИАНТЫ СРАВНЕНИЯ АРнольДА. Более сильное условие $\mathrm{in}_{*}[\mathbb{R} A]$ $=0$ в $H_{1}(\mathbb{R} X ; \mathbb{Z})$ позволяет выбрать в качестве коэффициентов $x_{i j}$ в $(4.2 .1)$ целье числа, и из 4.2.2 следует, что $\chi(B)=\xi_{+} \circ \xi_{-} \bmod 4$, где $B$ с $\partial B=\mathbb{R} A$ есть объединение компонент $C_{i j}$ с нечетными $x_{i j}$. С другой стороны, если $\operatorname{in}_{*}[\mathbb{R} A]=0$ в $H_{1}(\mathbb{R} X)$ и $B-$ произвольная часть поверхности $\mathbb{R} X$ с $\partial B=\mathbb{R} A$, то $\chi(B)=\mathscr{P}(\xi)-\chi(A)-\frac{1}{2}[A]^{2} \bmod 4$, 
где $\mathscr{P}$ - квадрат Понтрягина, а $\xi \in H_{2}(X)$ - класс, определяемьй циклом $A_{+}+B$. Такая часть $B$ с $\partial B=\mathbb{R} A$ существует, например, если in $*$. $A]=0 \in H_{2}(X)$, и в этом случае $\chi(B)=\mathscr{P}(\xi) \bmod 4$. Если, кроме того, $H_{1}(X)=0$, то $X$ является $\mathbb{Z}_{2}$-Галуа максимальной и $\xi=\frac{1}{2} A+\mathrm{bv}_{2} w_{1}(B)$, где $B$ - половина поверхности $\mathbb{R} X$, соответствуюшая двулистному накрытию; поэтому $\mathscr{P}(\xi)$ можно вычислить, используя форму Понтрягина-Виро.

Другое обобщение формулы Рохлина, полученное Ж.-И. Вельшингером [124], использует предположение: $\operatorname{in}_{*}[\mathbb{R} A] \in 2 l$ in $_{*}\left[H_{1}(\mathbb{R} A ; \mathbb{Z})\right] \subset H_{1}(\mathbb{R} X ; \mathbb{Z}), l \in \mathbb{Z}$. (В действительности, предположение в [124] является немного более общим.) Оно объединяет первоначальную формулу Рохлина, сравнение Арнольда и некоторые сравнения Михалкина [81]. Доказательство использует каноническое квадратичное отображение $\mathscr{P}_{\text {conj }}: H_{2}(X ; \mathbb{Z} / 2 l \mathbb{Z}) \rightarrow \mathbb{Z} / 4 l \mathbb{Z}$, являюшееся расширением редукции скрученной квадратичной формы $H_{2}(X ; \mathbb{Z}) \rightarrow \mathbb{Z}, x \mapsto x \circ \operatorname{conj} x$.

При указанном предположении сушествуют такие целые $x_{i j}, i=1, \ldots, n, j=$ $1, \ldots, m_{i}$, что $\left[\mathbb{R} A_{+}\right]=\partial \sum x_{i j}\left[C_{i j}\right] \bmod 2 l$. Обозначим через $x$ образ класса $\sum x_{i j}\left[C_{i j}\right]$ в $H_{2}(X, \mathbb{R} A ; \mathbb{Z})$. Существуют единственные классы $\xi_{+}, \xi_{-} \in H_{2}(X ; \mathbb{Z} / 2 l \mathbb{Z})$, переходяшие соответственно в $\left[A_{+}\right]-x,\left[A_{-}\right]+x$ при гомоморфизме релятивизации. Как и ранее, $\xi_{+}+\xi_{-}=[A]$.

4.2.5. ТЕОРемА. Если $A$ - разбивающая кривая $с \operatorname{in}_{*}[\mathbb{R} A] \in 2 l \operatorname{in}_{*}\left[H_{1}(\mathbb{R} A ; \mathbb{Z})\right]$, $l \in \mathbb{Z}, m o$

$$
\mathscr{P}_{\text {conj }}\left(\xi_{+}\right)=-\sum_{i, j=1}^{n, m_{i}} x_{i j}^{2} \chi\left(C_{i j}\right) \bmod 4 l .
$$

В некоторых случаях, например, если $H_{2}(X ; \mathbb{Z})$ порождается вешественными алгебраическими кривыми с известным расположением относительно $\mathbb{R} A$, элемент $\xi_{+}$ можно явно выгислить в терминах комплексной ориентации кривой $\mathbb{R} A$. Тогда 4.2 .5 дает новые ограничения на комплексные ориентации. Точные формулировки можно найти в [124].

4.3. Формулы присоединения. Формула Рохлина для комплексных ориентаций тесно связана с обобщенной формулой присоединения Бишопа-Лая, указанной ниже: они обе подсчитьвают алгебраическое число виртуальных двойных точек и, примененные в одной и той же ситуации, дают одинаковые результаты; более того, их геометрические доказательства аналогичны.

Традиционная формула присоединения для неособой кривой $D$ на неособой поверхности $X$ имеет вид:

$$
2 g(D)-2=[D]^{2}+\left[K_{X}\right] \circ[D]
$$

или, в эквивалентной записи,

$$
\chi(D)+D^{2}=c_{1}(X) \cap D .
$$

Если $D$ - особая кривая, дефект $\frac{1}{2}\left(\chi(D)+D^{2}-c_{1}(X) \cap D\right)$ можно интерпретировать как виртуальное число двойных точек кривой $D$.

Эту формулу можно легко обобщить с алгебраических кривых на погруженные вешественные двумерные многообразия (см. [73], [32]). Пусть $F$ - вешественное ориентированное компактное двумерное многообразие (возможно, несвязное и с краем) 
и $f: F \rightarrow X-$ погружение в комплексную поверхность $X$. Если $\partial F \neq \varnothing$, мы предполагаем, что либо $f$ не имеет комплексных точек на $\partial F$ (точка $x \in F$ называется комплексной, если $d f\left(T_{x} F\right) \subset T_{f(x)} X=\mathbb{C}^{2}$ есть комплексная прямая), либо все точки края $\partial F$ комплексные. Выберем следуюшим образом невырожденное векторное поле $n: \partial F \rightarrow f^{*}(T X)$, нормальное к $\partial F$ : если $f$ не имеет комплексных точек на $\partial F$, возьмем $n: \partial F \rightarrow T F \subset f^{*}(T X)$ нормальным к $\partial F$ на $F$; в противном случае $T F \subset f^{*}(T X)$ есть комплексное линейное подрасслоение расслоения $f^{*}(T X)$ над $\partial F$, и мы возьмем $n: \partial F \rightarrow f^{*}(T X)$ нормальным к $T F$.

Обозначим через $\nu(f) \in \mathbb{Z}$ препятствие к продолжению поля $\sqrt{-1} n$ до векторного поля, нормального к $F$, и через $c_{1}(f) \in \mathbb{Z}$ - препятствие к продолжению пары $(\tau, n)$, где $\tau$ - невырож денное касательное поле на $\partial F$, до поля комплексных 2-реперов в $f^{*}(T X)$ над $F$. Последнее число есть индекс Маслова поля 2-реперов относительно перепонки $F$. Если $\partial F=\varnothing$, то $\nu(f)$ - нормальное число Эйлера поверхности $F$ в $f^{*}(T X)$ и $c_{1}(f)=c_{1}(X) \cap f_{*}[F]$. В этих обозначениях формула Бишопа-Лая утверждает, что

$$
\chi(F)+\nu(f)-c_{1}(f)=2 d_{-}(f), \quad \chi(F)+\nu(f)+c_{1}(f)=2 d_{+}(f),
$$

где $d_{ \pm}(f)$ определяются числом комплексных точек погружения $f$. Точнее, для общего отображения $f$ положим $d_{ \pm}(f)=e_{ \pm}(f)-h_{ \pm}(f)$, где $e_{ \pm}$(соответственно $h_{ \pm}$) - числа положительных и отрицательных эллиптических (соответственно гиперболических) точек отображения $f$. В обшем случае $d_{+}(f)$ и $d_{-}(f)$ можно определить как индексы пересечения образа поверхности $F$ в гауссовом расслоении на ориентированные вешественные двумерные плоскости в $f^{*}(T X)$ с циклом комплексных прямых, наделенных их комплексной или соответственно антикомплексной ориентацией. Если компонента края $\partial F$ состоит из комплексных точек, ее надо сначала отодвинуть от цикла в направлении касательного векторного поля $\tau^{*} \otimes n+\sqrt{-1} \tau^{*} \otimes \sqrt{-1} n$, определяемом вещественными двумерными плоскостями $(\tau, n)$. Для общего отображения $f$ числа $e_{+}$и $h_{+}$(соответственно $e_{-}$и $h_{-}$) есть числа положительных и отрицательных точек пересечения поверхности $F$ с указанным комплексно (соответственно антикомплексно) ориентированным циклом. Определение эллиптических и гиперболических точек в локальных координатах можно найти, например, в [32].

4.3.3. Определение чисел $\nu(f)$ и $c_{1}(f)$ и формула Бишопа-Лая распространяются на более широкий класс поверхностей: можно считать, что $F$ и $f: F \rightarrow X$ сшиты (вдоль компонент края) из поверхностей $F_{i}$ и погружений $f_{i}: F_{i} \rightarrow X$, удовлетворяющих вышеуказанным условиям, так, что любые два соседних куска примыкают друг к другу нормально в $X$. (Мы не накладываем никаких ограничений на поля вдоль сшитых краев, поскольку не используем формулы сложения.) Заметим также, что если $F$ замкнута, алгебраическое число $i(f)$ виртуальных двойных точек отображения $f$ задается равенством

$$
i(f)=\frac{1}{2}\left(\left(f_{*}[F]\right)^{2}-\nu(f)\right)=\frac{1}{2}\left(\chi(F)+\left(f_{*}[F]\right)^{2} \pm c_{1}(f)\right)-d_{ \pm}(f) .
$$

Следуюшее утверждение сразу следует из (4.3.2). 
4.3.5. Пусть $F$ - замкнутое ориентированное вещественное двумерное многообразие, $X$ - комплексная поверхность и $f: F \rightarrow X-$ кусочно гладкое отображение такое же, как и в 4.3.3, причем его образ не имеет отрицательньх комплексных точек. Тогда

$$
\chi(F)+\nu(f)=c_{1}(f) .
$$

4.3.6. СЛЕДСТВИЕ. $В$ обозначениях $n$. 4.2 .2

$$
\xi_{ \pm}^{2}=\frac{1}{2}[A]^{2}-d^{+-}-\sum_{i, j=1}^{n, m_{i}} x_{i j}^{2} \chi\left(C_{i j}\right) .
$$

ДокАЗАТЕльство. Применим 4.3.5 дважды: сначала к $\xi$ (или к каждому его гладкому куску), а затем к $A$. (Ясно, что тот же результат следует из 4.2 .2 и 4.2 .3 , ср. с началом этого раздела.)

В случае плоских кривых 4.3.6 преврашается в формулу Рохлина.

4.4. Применение неравенства присоединения. Пусть кривая $A \subset X$ - такая же, как в п. 4.2. Предположим, что in $*\left[\mathbb{R} A_{+}\right]=0 \in H_{1}(\mathbb{R} X ; \mathbb{Z})$ и обозначим через $x_{i j}$ целые числа, определяемые равенством (4.2.1). Цикл $\xi_{+}$, см. п. 4.2, можно представить в виде $f_{*}[F]$ для некоторого отображения $f: F \rightarrow X$ и некоторой поверхности $F$, сшитой из $A_{+}$и $\left|x_{i j}\right|$ копий поверхностей $C_{i j}$. Такой цикл не имеет отрицательных комплексных точек, и потому в силу (4.3.4) и 4.3.5 число $i_{+}\left(f^{\prime}\right)$ положительных двойных точек общей бифуркации $f^{\prime}$ отображения $f$ удовлетворяет неравенству присоединения

$$
i_{+}\left(f^{\prime}\right) \geqslant \frac{1}{2}\left(\chi(F)+\left(f_{*}[F]\right)^{2}-c_{1}(f)\right) .
$$

Результаты, полученные с использованием этого неравенства, слабее, чем формула Рохлина (которая в этом случае равносильна формуле Бишопа-Лая). Однако, как заметил Г. Б. Михалкин [82], можно получить новые запреты для приводимых вешественных кривых, применяя неравенство присоединения к подобным смешанным циклам с обращенной ориентацией некоторых голоморфных кусков. (Для простоты мы игнорируем более громоздкий случай, когда неприводимые компоненты имеют вешественные точки пересечения; с другой стороны, мы работаем с кривыми на произвольных кэлеровых поверхностях.)

Итак, зафиксируем целое $s \geqslant 1$ и положим $f^{\prime}: F \rightarrow X$ равным $f$ на всех $C_{i j}$ и всех $A_{+}^{k}$ с $k>s$ и равным conjof на всех $A_{+}^{k} \mathrm{c} 1 \leqslant k \leqslant s$. Обозначим через $d_{1}, d_{2} \in H_{2}(X ; \mathbb{Z})$ элементы, реализуемые соответственно поверхностями $\bigcup_{k>s} A_{k}$ и $\bigcup_{k \leqslant s} A_{k}$, и положим $x^{++}$равным алгебраическому индексу пересечения цепей $\bigcup_{k>s} A_{+}^{k}$ и $\bigcup_{k \leqslant s} A_{+}^{k}$ (напомним, что согласно сделанным предположениям, см. п. 4.2, границы этих цепей не пересекаются).

4.4.1. Теорема. Пусть кривая $A \subset X$ такая жсе, как в $n .4 .2, u \operatorname{in}_{*}\left[\mathbb{R} A_{+}\right]=0$ в $H_{1}(\mathbb{R} X ; \mathbb{Z})$. Если $X$ кәлерова и $\omega \cap\left(d_{1}-d_{2}\right)>0$, где $\omega-$ кәлеров класс, то

$$
j_{-} \geqslant x^{++}+\frac{1}{2}\left(c_{1}(X) \cap d_{2}-d_{1} \circ d_{2}\right),
$$

где $j_{-}=\sum \frac{1}{2}\left|x_{i j}\right|\left(\left|x_{i j}\right|-1\right)$, причем сумма берется по всем $\left|x_{i j}\right| \geqslant 2$, отвечаюшим компонентам $C_{i j}$, гомеоморфным кругу. 
ДокАЗАТЕльство. Это неравенство есть разность между равенством 4.3.5, примененным к $f$, и неравенством присоединения, примененным к обычному лагранжеву возмущению отображения $f^{\prime}$, представляющему собой погружение с $j_{-}+\frac{1}{2}\left(d_{1} \circ d_{2}\right)-$ $x^{++}$отрицательными двойными точками.

4.4.2. С ЛЕДСТВИЕ. Пусть $X, d_{1}, d_{2}$ - такие жее, как и вышие, $u \omega \cap\left(d_{1}-d_{2}\right)>0$ (см. 4.4.1). Предположси, что инвариантное относительно сопј ${ }_{*}$ подпространство в $\mathrm{H}_{2}(X ; \mathbb{Q})$ порождается фундаментальными классами ориентируемых компонент поверхности $\mathbb{R} X$ и что для любого $k$ каждая компонента связности кривой $\mathbb{R} A^{k}$ ограничивает круг. Тогда

$$
j_{-} \geqslant z^{+}-z^{-}+\frac{1}{2} c_{1}(X) \cap d_{2}-\frac{1}{4}\left(d_{1} \circ d_{2}\right),
$$

где $z^{ \pm}$- числа положительных и отрицательных инбективных пар овалов таких, что один из овалов лежит в $A_{k} c k \leqslant s$, а другой - в $A_{k} c k>s$. (Для того чтобы определить ингективную пару овалов, надо для каждой компоненты связности кривой $\mathbb{R} A$ выбрать и зафиксировать круг, ограниченный этой компонентой.)

ДокАЗАТЕльство. Построим циклы Рохлина, дополнив $\bigcup_{k>s} A_{+}^{k}$ и $\bigcup_{k \leqslant s} A_{-}^{k}$ кругами. Тогда $x^{++}$можно найти, вычисляя пересечение этих циклов двумя способами: гомологически (применяя 4.2.4) и геометрически.

Следующий пример применения п. 4.4.2 (вместе с формулой Рохлина) также принадлежит Михалкину. Пусть $A$ - вешественная плоская кривая степени $2 k$ с вешественной схемой $\left\langle\frac{3}{2} k(k-1)\right\rangle \sqcup 1\left\langle\frac{1}{2}(k-1)(k-2)\right\rangle$ (схема Харнака) и $C$ - вещественная коника с $\mathbb{R} C \cap \mathbb{R} A=\varnothing$. Тогда не более $(k-2)$ внутренних овалов кривой $\mathbb{R} A$ лежат внутри овала кривой $\mathbb{R} C$.

4.5. Формула Оревкова. С. Ю. Оревков [92] заметил, что если плоская $M$-кривая степени $d$ имеет гнездо глубины $k-1, k=[d / 2]$, формула Рохлина распадается на два отдельных равенства. Для такой кривой непустые овалы содержатся один в другом и потому разбиваются на положительнье и отрицательные (например, относительно самого внешнего овала гнезда). Обозначим через $\pi_{+}^{+}$и $\pi_{+}^{-}$(соответственно $\pi_{-}^{+}$и $\pi_{-}^{-}$) числа положительных и отрицательных инъективных пар, внутренний овал которых пуст, а внешний положителен (соответственно отрицателен).

4.5.1. ФормУЛА ОревКова. Для любой плоской $M$-кривой степени $2 k$ с әнездом глубины $k-1$ справедливы равенства

$$
\pi_{+}^{-}-\pi_{+}^{+}=k_{+}^{2}, \quad \pi_{-}^{+}-\pi_{-}^{-}=k_{-}^{2},
$$

әде $k_{+} u k_{-}-$числа положстельных и отрицательных непустых овалов.

Указанная формула и аналогичная ей формула для кривых нечетной степени дают новую информацию о комплексных ориентациях для степеней $\geqslant 7$. Обе формулы распространяются на любые разбивающие кривые.

Первоначальное доказательство (см. [92]) основано на подсчете коэффициентов самозацеплений кос, высекаемых кривой $\mathbb{C} A$ на границе двух четырехмерных шаров, образованных мнимыми точками мнимых прямых, проходящих через точку внутри самого внутреннего овала гнезда. Ж.-И. Вельшингер [125] заметил, что формулу Оревкова 
можно также получить из формулы присоединения, примененной к соответствующим смешанным особым циклам, и обобшил эту формулу на кривые, лежашие на поверхностях, расслоенных на коники. Для простоты мы сформулируем его результат для геометрически линейчатых поверхностей, т.е. для расслоений на неособые коники.

Пусть $\pi: X \rightarrow B$ - голоморфное эквивариантное отображение вешественной поверхности на вещественную кривую. Предположим, что $B$ - разбиваюшая кривая, а каждый слой $F$ отображения $\pi$ - гладкая неприводимая рациональная кривая. Предположим, кроме того, что $\pi$ имеет вещественное сечение $E$. Фундаментальные классы кривых $F$ и $E$ являются образуюшими групшы $H_{2}(X ; \mathbb{Z})$.

Обозначим через $B_{1}, \ldots, B_{p}$ компоненты связности кривой $\mathbb{R} B$ и через $X_{1}, \ldots, X_{p}$ - их прообразы в $\mathbb{R} X$. (Каждая поверхность $X_{j}$ есть тор или бутылка Клейна.) Выберем половину $B_{+}$кривой $B$ и наделим $\mathbb{R} B$ соответствуюшей комплексной ориентацией.

Пусть $A \subset X$-неособая неприводимая разбивающая вешественная $(M-2 r)$-кривая со стягиваемьм овалом на каждой вешественной компоненте поверхности $X$ (последнее условие не существенно для 4.5.2). Выберем половину $A_{+}$кривой $A$ и наделим $\mathbb{R} A$ соответствующей комплексной ориентацией. Для каждого $i, 1 \leqslant i \leqslant p$, рассмотрим компоненты кривой $\mathbb{R} A$, которые проектируются на $B_{i}$ с положительной (соответственно отрицательной) степенью, и обозначим через $n_{i}^{+}$(соответственно $n_{i}^{-}$) их общую степень. Для каждого $i$ сушествует такое положительное целое $k_{i}$, что $n_{i}^{+}+n_{i}^{-}+2 k_{i}=[A] \circ[F]$. Если $k_{i}=1$ для всех $i, 1 \leqslant i \leqslant p$, будем говорить,

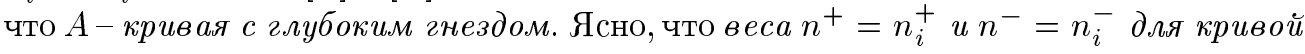
с глубоким гнездом не зависят от $i$.

Всюду далее будем считать, что $A$ - кривая с глубоким гнездом. Тогда каждый вешественньй слой отображения $\pi$ имеет не более двух общих с кривой $A$ мнимых точек. Если вешественный слой $F$ имеет две общие с кривой $A$ мнимые точки, выберем точку $x \in \mathbb{R} F$, не лежашую на $E$. Такой выбор можно сделать непрерывным. Более того, если все точки поворота (т.е. точки касания кривой $\mathbb{R} A$ со слоем) не лежат на $E$, сушествует канонический выбор: проведем окружность $\gamma$ в $F$ через две указанные мнимые точки и через точку кривой $E$ и возьмем в качестве $x$ точку пересечения этой окружности с $\mathbb{R} F$, отличную от точки на $E$. Выбранные точки образуют конечный набор дуг $S_{j}$, каждая из которых соединяет две точки поворота. Объединение дуг $S_{j}$ дополняется до замкнутой, не обязательно связной или простой, кривой $\Gamma^{+}$ (соответственно $\Gamma^{-}$) дугами кривой $\mathbb{R} A$, проектирующимися на $\mathbb{R} B$ с положительной (соответственно отрицательной) локальной степенью. Комплексная ориентация кривой $\mathbb{R} A$ определяет коориентацию дуги $S_{j}$ в ее граничных точках; она продолжается до непрерьвной коориентации дуги $S_{j}$. Обозначим продолженную коориентацию через $\omega$. Не умаляя общности, можно считать, что пересечения внутренностей дуг $S_{j}$ c $\mathbb{R} A$ трансверсальны; припишем каждой точке такого пересечения знак +1 , если $\omega$ совпадает с ориентацией кривой $\mathbb{R} A$, и -1 в противном случае. Пусть $\Lambda^{ \pm}-$сумма этих знаков по всем двойным точкам кривой $\Gamma^{ \pm}$.

Чтобы сделать $\Lambda^{ \pm}$более инвариантными, удалим зигзаги на $\mathbb{R} A$ (каждьй зигзаг меняет одно из чисел $\Lambda^{ \pm}$на $n^{ \pm}-1$ или 1 в зависимости от того, пересекает он $\mathbb{R} E$ или нет). После этого $\Lambda^{ \pm}$будут сохраняться при изотопиях неориентируемых компонент поверхности $\mathbb{R} X$, тождественных на $\mathbb{R} E$ и на каждой компоненте кривой $\mathbb{R} A$, пересекающей $\mathbb{R} E$. Более общо, $\Lambda^{ \pm}$сохраняются при изотопии неориентируемой ком- 
поненты поверхности $\mathbb{R} X$, тождественной на $\mathbb{R} E$, если во время изотопии точки поворота не пересекают $\mathbb{R} E$ (каждое пересечение меняет оба числа $\Lambda^{ \pm}$на $n^{ \pm}$). Изотопия ориентируемой компоненты поверхности $\mathbb{R} X$ сохраняет $\Lambda^{ \pm}$, если, например, во время изотопии (так же, как и в начальном положении) $\mathbb{R} A$ не пересекает $\mathbb{R} E$.

4.5.2. ТЕОРЕМА. При сделанных выше предположсениях существуют такие иелье числа $\mu^{ \pm}, g^{ \pm}, a^{ \pm}$, что

$$
\Lambda^{ \pm}+n^{ \pm} a^{ \pm}+\mu^{ \pm}-g^{ \pm}=\frac{1}{2}\left(\chi(B)-n^{ \pm} E^{2}\right)\left(n^{ \pm}+1\right)
$$

$u$

$$
\begin{gathered}
g^{ \pm} \geqslant c^{ \pm}, \quad g^{+}+g^{-}+2-\mu^{+}-\mu^{-}=c^{+}+c^{-}+2 r, \\
\quad \mu^{ \pm} \geqslant 1, \quad \mu^{+}+\mu^{-} \leqslant p+1, \\
a^{+}+a^{-}=a, \quad a^{ \pm}+E^{2}\left(n^{ \pm}+1\right)=\mathbb{R} E \circ \Gamma^{ \pm} \bmod 2, \\
\left|\mathbb{R} E \cap \Gamma^{ \pm}\right| \leqslant a+E^{2}\left(n^{ \pm}+1\right) \leqslant a^{ \pm}+E^{2} b-\left|\mathbb{R} E \cap \Gamma^{\mp}\right|,
\end{gathered}
$$

причем $a[F]+b[E]=[A]$, a $c^{+}, c^{-}-$числа компонент кривой $\mathbb{R} A$, проектирующихся в компоненту кривой $\mathbb{R} B$ с положительной (соответственно отрицательной) степенью.

ДокАЗАТЕЛЬСтво. Рассмотрим поверхность

$$
W^{ \pm}=\left(A^{ \pm} \cap \pi^{-1}\left(B^{+}\right)\right) \cup\left(A^{\mp} \cap \pi^{-1}\left(B^{-}\right)\right)
$$

и заполним ее дыры вложенными кругами, лежащими в прообразах кривой $\mathbb{R} B$, диаметрами которых являются сегменты $S_{j}$ (например, можно использовать дуги окружностей, указанных в построении дуг $S_{j}$ ). Утверждение теоремы следует из формулы присоединения 4.3.5, примененной к полученному особому циклу $\Sigma^{ \pm}$, если использовать равенства $\nu=\left(\Sigma^{ \pm}\right)^{2}+2 \Lambda^{ \pm}, \Sigma^{ \pm} \circ F=n^{ \pm}+1$, ввести $a^{ \pm}$, исходя из формулы $\left[\Sigma^{ \pm}\right]=a^{ \pm}[F]+\left(n^{ \pm}+1\right)[E]$, и взять в качестве $g^{ \pm}$и $\mu^{ \pm}$род и число компонент связности поверхности $W^{ \pm}$.

4.5.3. СлЕДСтвиЕ. В условиях $n$. 4.5.2 если $A$ не пересекает $E$, то

$$
\Lambda^{ \pm}+\mu^{ \pm}-g^{ \pm}=\frac{1}{2}\left(\chi(B)+E^{2} n^{ \pm}\right)\left(n^{ \pm}+1\right)
$$

ЗАмЕЧАНИЕ. Чтобы получить 4.5.1 из 4.5.3, раздуем точку внутри самого внутреннего овала некоторого гнезда глубины $k-1$; тогда $\chi(B)=2, E^{2}=-1, \mu^{ \pm}=1$, $g^{ \pm}=K_{ \pm}$и $\Lambda^{ \pm}=\Pi_{ \pm}^{-}-\Pi_{ \pm}^{+}$, где $K$ и П отличаются от $k$ и $\pi$ в 4.5.1 тем, что самьй внутренний овал выбранного гнезда дает вклад в $K$ и не дает в П и в то же время не дает вклад в $k$ и дает в $\pi$.

4.6. Теорема Безу. Теорема Безу играет важную роль в теории вещественных плоских проективных алгебраических кривых. Для произвольной поверхности $X$ комплексная форма этой известной теоремы утверждает, что если кривые $A, B \subset X$ не имеют общих компонент, то число их точек пересечения с учетом кратностей равно $[A] \circ[B]$. Важным моментом является то, что кратность точки пересечения 
двух аналитических кривых, не имеющих общих компонент, - целое положительное число. Это дает вешественный вариант теоремы Безу (ср. замечание в А.2): ecли $X$ вещественная поверхность и $A, B \subset X$ - вещественные кривые без общих компонент, то число точек пересечения кривых $\mathbb{R} A$ u $\mathbb{R}$ с учетом их кратностей не превосходит $[A] \circ[B]$ и сравнимо с $[A] \circ[B] \bmod 2$.

Одно из типичных применений теоремы Безу - проведение вспомогательной кривой $D$ через определенное число подходящим образом выбранных точек и оценка числа точек пересечения кривой $\mathbb{R} D$ с данной кривой $\mathbb{R} A$ (именно таким способом Харнак получил свою оценку числа вешественных компонент плоской кривой). Иногда требуется, чтобы кривая $\mathbb{R} D$ была связной или, более общо, чтобы все точки пересечения лежали на одной компоненте кривой $\mathbb{R} D$. Простейший способ обеспечить это - рассматривать рациональные кривые.

Таким образом, для применения теоремы Безу на произвольной поверхности $X$ нужно, во-первых, выяснить, можно ли данный класс $x \in H_{2}(X ; \mathbb{Z})$ реализовать формальной линейной комбинацией аналитических кривых, и, во-вторых, найти размерность пространства таких кривых и их род. Ответ на первый вопрос в случае, когда $X$ проективна, дается теоремой Лефшеца-Ходжа (см. [68]): гомологический класс $x \in H_{2}(X ; \mathbb{Z})$ можсно реализовать формальной линейной комбиначией алгебраических кривых на $X$ тогда и только тогда, когда класс, двойственный по Пуанкаре классу $x$, принадлежст группе $H^{1,1}(X)$ (см. 2.4). Несмотря на то, что для поверхностей размерность $h^{1,1}(X)=\operatorname{dim} H^{1,1}(X)$ является топологическим инвариантом: $h^{1,1}(X)=\sigma^{-}(X)+1$, расположение подгруппы $H^{1,1}$ в $H^{2}(X ; \mathbb{Z})$ зависит от комплексной структуры на $X$ и может меняться при варьировании поверхности $X$ в пределах ее деформационного типа. Поэтому в общем случае можно лишь утверждать, что рациональные кратные класса гиперплоского сечения (те, которые являются целочисленньми классами) можно реализовать комбинациями алгебраических кривых. Однако, если $h^{1,1}(X)=b_{2}(X)$, т.е. $\sigma^{+}(X)=1$, все классы в $H_{2}(X ; \mathbb{Z})$ являются алгебраическими. Примеры - рациональные поверхности и поверхности Энриквеса.

Размерность линейной системы $|D|$ в определенных случаях можно оценить, используя теорему Римана-Роха:

$$
\operatorname{dim}|D|+\operatorname{dim}\left|K_{X}-D\right| \geqslant \frac{1}{2}\left([D]^{2}-\left[K_{X}\right] \circ[D]\right)+p_{a}(X)-1,
$$

где $p_{a}(X)=\frac{1}{2}\left(\sigma^{+}(X)-b_{1}(X)+1\right)$ есть арифметический род поверхности $X$. Если $D$ обилен (напомним, что для эффективного дивизора на поверхности это эквивалентно тому, что $[D]^{2}>0$ и $[D] \circ\left[D^{\prime}\right] \geqslant 0$ для всех неприводимых компонент $D^{\prime}$ дивизора $\left.D\right)$, то $\left|K_{X}-D\right|=\varnothing$ и в 4.6.1 достигается равенство:

$$
\operatorname{dim}|D|=\frac{1}{2}\left([D]^{2}-\left[K_{X}\right] \circ[D]\right)+p_{a}(X)
$$

Следующее утверждение легко вывести из 4.6.2.

4.6.3. ПРЕДЛОЖЕНИЕ. Пусть $D$ - обильный вещественный дивизор и $s_{D}=$ $\frac{1}{2}\left([D]^{2}-\left[K_{X}\right] \circ[D]\right)+p_{a}(X)$. Тогда через любие $s_{D}$ точек мохсно провести 
вещественную кривую, принадлежсащую $|D|$. Более того, для любого $\left(s_{D}-s\right)$-точечного множсества $S, s \geqslant 0$, вещественные кривые из $|D|$, содержащие $S$, образуют вещественную линейную систему размерности $\geqslant s$.

К сожалению, этот результат недостаточен даже для обобшения хорошо известных неравенств Гильберта на случай большего числа гнезд. Трудность состоит в том, что число компонент связности кривой, даваемой предложением 4.6.3, нелегко проконтролировать. В обшем случае можно лишш утверждать, что это число лежит между 1 и $2+\frac{1}{2}\left([D]^{2}+\left[K_{X}\right] \circ[D]\right)$, т.е. границей Харнака. Поэтому, кроме того, было бы полезно знать, через какое число точек можно провести компоненту связности кривой, принадлежащей $|D|$. (Этот и другие подобные вопросы, более или менее явно поднятые в [99], по-видимому, представляют интерес независимо от неравенств для гнезд.)

Обозначим через $c(D)$ максимальное число $c$ такое, что через любые $c$ точек поверхности $\mathbb{R} X$, находящихся в общем положении, можно провести вещественную кривую, принадлежащую $|D|$ и имеющую связное множество вешественных точек. Обозначим, далее, через $c^{\prime}(D)$ максимальное число $c$ такое, что через любые $c$ точек поверхности $\mathbb{R} X$ (не обязательно в общем положении) можно провести компоненту связности множества вешественных точек вешественной кривой, взятой из $|D|$.

4.6.4. ПРЕДЛОЖЕНИЕ. $c^{\prime}(D) \geqslant c(D)$ для любого дивизора $D$.

4.6.5. Лемма. Пусть $\left\{F_{n}\right\}$ - последовательность непрерывных функиий на компактном хаусдорфовом пространстве $Y$, равномерно сходящаяся к функиии F. Если множсество нулей $Z_{n}$ функиии $F_{n}$ связно для каждого $n$, то множество нулей $Z$ функиии $F$ имеет компоненту связности, содержсащую $\bigcap_{n} Z_{n}$.

ДокАЗАТЕЛьство. Пусть пересечение $\bigcap_{n} Z_{n}$ непусто. Оно содержится в $Z$. Выберем точку $z \in \bigcap_{n} Z_{n}$ и открыто-замкнутое подмножество $C$ в $Z$, содержашее $z$. Поскольку $Z$ замкнуто в $Y$, множества $C$ и $Z \backslash C$ тоже замкнуты и, следовательно, разделяются окрестностью $U$ (т.е. $U \supset C$ и $\mathbb{C} U \cap(Z \backslash C)=\varnothing)$.

Так как сходимость $F_{n} \rightarrow F$ является равномерной, сушествует такой номер $N$, что $Z_{n} \subset U \cup(Y \backslash \mathbb{C} l U)$ для всех $n>N$. Тогда, поскольку все $Z_{n}$ связны и пересекают $C \subset U$, каждое $Z_{n}$ с $n>N$ содержится в $U$, и, следовательно, $\bigcap_{n} Z_{n}$ содержится в $C=U \cap Z$. Остается заметить, что в компактном хаусдорфовом пространстве пересечение всех открыто-замкнутых подмножеств, содержаших данную точку, есть компонента связности этой точки.

ДОКАЗАТЕЛЬСТВО ПРЕДЛОЖЕНИЯ 4.6.4. Пусть $c$ - такое число, что через любые $c$ точек в общем положении на $\mathbb{R} X$ можно провести вешественную алгебраическую кривую из $|D|$ со связньм множеством вешественных точек. Нужно доказать, что любое $c$-точечное множество в $\mathbb{R} X$ содержится в компоненте связности вещественной кривой из $|D|$.

Рассмотрим $c$-точечное множество $\Sigma \subset \mathbb{R} X$. По предположению существует последовательность $c$-точечных множеств $\left\{\Sigma_{n}\right\}$ в $\mathbb{R} X$, сходящаяся к $\Sigma$ и такая, что каждое $\Sigma_{n}$ лежит на связной вещественной кривой $\mathbb{R} A_{n}$ из $|D|$. Пусть $\xi$ есть $\mathbb{R}$-расслоение над $\mathbb{R} X$, соответствующее линейной системе $|D|$. Его тензорный квадрат, как квадрат любого $\mathbb{R}$-расслоения, тривиален. Пусть $F_{n}, n \geqslant 1,-$ вешественное сечение расслоения $\xi$, представляющее кривую $A_{n}$. Поскольку пространство сечений конечномерно, 
после соответствуюшей нормировки последовательность $\left\{F_{n}\right\}$ будет содержать сходящуюся подпоследовательность. Как обычно, можно считать, что $\left\{F_{n}\right\}$ сама сходится. Остается применить лемму 4.6 .5 к $F_{n}^{2}$, рассматриваемым как функции на $\mathbb{R} X$.

Следуюшее свойство аддитивности сразу следует из определений.

4.6.6. ПРЕДЛОЖЕНИЕ. Для любъх дивизоров $D_{1}, D_{2}$

$$
c\left(D_{1}+D_{2}\right) \geqslant c\left(D_{1}\right)+c\left(D_{2}\right)-1 \quad u \quad c^{\prime}\left(D_{1}+D_{2}\right) \geqslant c^{\prime}\left(D_{1}\right)+c^{\prime}\left(D_{2}\right)-1
$$

Упомянем о некоторых других проблемах, связанных с этой темой. Во-первых, естественно рассматривать кривые с ограниченным числом компонент. Тогда $c(D)$ становится первым членом $c_{1}(D)$ большего семейства характеристик $c_{\leqslant k}(D)$, определяемых аналогично $c(D)$ с заменой требования связности кривой условием иметь не более $k$ компонент связности. Во-вторых, можно рассматривать минимакс $\nu(r, D)$ и максимин $\mu(r, D)$ числа компонент связности в линейной подсистеме данной размерности $r$ в $|D|$. Другая возможность - рассматривать иные топологические характеристики линейных систем, скажем, типовые числа Морса и т. д.

4.6.7. ОЦенка МАренА. Если дивизор D очень обилен и число $\chi(X)+D^{2}$ нечетно, любой общий пучок вещественных кривых в $|D|$ содержит хотя бъ одну $(M-j)$-кривую с некоторьм $j \geqslant 3$.

Этот результат можно переформулировать так: $\mu(1, D) \leqslant M(D)-3$, где $M(D)-$ число Харнака $2+\frac{1}{2}\left([D]^{2}+\left[K_{X}\right] \circ[D]\right)$.

4.6.8. УТВЕРЖДЕНИЕ КЛЕЙНА. Если вещественная кривая типа I подвергается морсовской перестройке при переходе через невырожденную двойную точку, то число ее компонент связности не увеличивается.

Заметим, что число компонент связности такой кривой не обязательно меняется, cр. 4.6.9 ниже. Утверждение Клейна, другими словами, означает, что для любого $q$ число компонент связности, рассматриваемое как кусочно постоянная функция на пространстве $\mathbb{R} C_{q}$ кривых степени $q$, достигает “локального максимума" на каждой компоненте пространства $\mathbb{R} C_{q} \backslash \mathbb{R} D_{q}$ (где $\mathbb{R} D_{q}$ - гиперповерхность особых кривых), образованной вешественньми кривыми типа I. В добавление к 4.6.7 и 4.6.9 можно показать, используя комплексные ориентации, что не существует стенки (т.е. старшего страта множества $\mathbb{R} D_{q}$ ), к которой примыкают две компоненты, образованные кривыми типа I. (Имеется одно исключение: $q=2$ и стенка, соответствующая выворачиванию овала наизнанку; однако эта стенка не разделяет две разные компоненты!)

ЗАмЕчАнИЕ. Утверждение 4.6.8 распространяется на поверхности (см. [119]) и на гиперповерхности в проективном пространстве (см. [54]).

ДокАЗАТЕЛЬСтво УТВЕРЖДЕНИЯ 4.6.8. Пусть $A_{t}, t \in[-\varepsilon, \varepsilon],-$ такое семейство вешественных кривых, что $A_{0}$ - кривая с невырожденной двойной точкой, кривые $A_{<0}$ принадлежат типу I и число компонент связности уменьшается при переходе от $\mathbb{R} A_{>0} \mathrm{k} \mathbb{R} A_{<0}$. Из последнего условия следует, что особая компонента связности множества $\mathbb{R} A_{0}$, поднятая в нормализацию кривой $A_{0}$, есть топологическая окружность. В частности, число вещественных компонент нормализации кривой $A_{0}$ равно числу 
компонент множества $\mathbb{R} A_{<0}$. $\mathrm{C}$ другой стороны, род кривой $A_{0}$ и род кривой $A_{\neq 0}$ отличаются на $1: g\left(A_{0}\right)=g\left(A_{\neq 0}\right)-1$. Поэтому в силу, например, сравнения Клейна (которое утверждает, что число вешественных компонент разбивающей кривой сравнимо с родом по модулю 2 , ср. введение) $A_{0}$ не принадлежит типу I. Следовательно, две комплексно сопряженные точки в $A_{0} \backslash \mathbb{R} A_{0}$ можно соединить путем в $A_{0} \backslash \mathbb{R} A_{0}$. Значит, такой путь существует и в каждой близкой кривой $A_{t}, t \neq 0$, что противоречит принадлежности кривых $A_{t} \mathrm{c} t<0$ типу I.

Для $(M-2)$-кривых утверждение Клейна можно усилить.

4.6.9. ПРЕДЛОЖЕНИЕ. Если две $(M-2)$-кривъе получаются друг из друга морсовской перестройкой при переходе через невырожденную двойную точку, то одна из них принадлежит типу I, а другая - типу II.

ДокАЗАТЕльство. Обозначим через $A_{t}, t \in[-\varepsilon, \varepsilon]$, семейство таких вешественных кривых, что $A_{0}-$ кривая с невырожденной двойной точкой и $A_{\neq 0}$ являются $(M-2)$-кривыми. Так как число компонент связности не меняется от $\mathbb{R} A_{>0} \mathrm{~K} \mathbb{R} A_{<0}$, особая компонента связности множества $\mathbb{R} A_{0}$, поднятая в нормализацию кривой $A_{0}$, преврашается в две топологические окружности. В частности, число вещественных компонент нормализации кривой $A_{0}$ больше на 1 числа компонент множества $\mathbb{R} A_{t}$ c $t \neq 0$. С другой стороны, $g\left(A_{0}\right)=g\left(A_{\neq 0}\right)-1$. Поэтому нормализация является $M$-кривой и, следовательно, принадлежит типу I, а вместе с ней и ее возмущение, согласованное с комплексной ориентацией (в то время как другое возмущение принадлежит типу II).

ДОКАЗАТЕЛЬСТво УТВЕРЖДЕНИЯ 4.6.7. Рассмотрим граф̆ Г с 4 вершинами, представляющими $M-,(M-1)$-кривые, $(M-2)$-кривые типа I и $(M-2)$-кривые типа II. Две вершины соединим ребром, если их можно представить примыкающими кривыми. В силу 4.6.8 и 4.6.9 Г имеет только три ребра: они соединяют вершины $M$ и $M-1$, $M-1$ и $M-2$ типа II, $M-2$ типа II и $M-2$ типа I. В частности, Г не содержит циклов нечетной длины.

Общий пучок вешественных кривых в $|D|$, рассматриваемый как прямая в соответствуюшем проективном пространстве, пересекает дискриминантную гиперповерхность особых кривых трансверсально, причем точки пересечения отвечают перестройкам вешественных частей кривых. Степень дискриминанта сравнима с $\chi(X)+D^{2}$ по модулю 2 . Поэтому если пучок не содержит $(M-j)$-кривую с $j \geqslant 3$, он определяет цикл нечетной длины в построенном выше граффе, что приводит к противоречию.

В заключение этого раздела мы используем предыдушие результаты для получения оценок числа овалов в гнездах. Первая теорема формулируется так, что она зависит только от нижней гранищы для $c^{\prime}(D)$.

4.6.10. ПРЕДЛОЖЕНИЕ. Если вещественная кривая $C$ имеет $c \leqslant c^{\prime}(D)$ таких дизбюнктных гнезд глубины $h_{1}, \ldots, h_{c}$, что ни один овал гнезд глубины $>1$ не охватывает все остальнье оваль әтих гнезд, то

$$
h_{1}+\cdots+h_{c}+\left[\frac{1}{2}\left(c^{\prime}(D)-c\right)\right] \leqslant \frac{1}{2} C \circ D .
$$


В частности, если $C$ имеет $c \leqslant c^{\prime}(D)-1$ таких дизгюнктных гнезд глубиньи $h_{1}, \ldots, h_{c}$, что ни один овал гнезд глубины > 1 не охватывает все остальные оваль әтих гнезд и

$$
h_{1}+\cdots+h_{c}+\left[\frac{1}{2}\left(c^{\prime}(D)-c\right)\right]=\left[\frac{1}{2} C \circ D\right] \text {, }
$$

то облединение этих гнезд исчерпьвает всю кривую.

ДокАЗАТЕльство. В каждом гнезде возьмем точку на самом внутреннем овале (или внутри него, если $h>1$ ) и еше $c^{\prime}(D)-c$ точек на одном из этих овалов. Проведем связную компоненту вешественной кривой из $|D|$ через $c^{\prime}(D)=c+\left(c^{\prime}(D)-c\right)$ полученных точек. Она пересекает исходную кривую не менее, чем в $2\left(h_{1}+\cdots+h_{c}\right)+r$ точках, где $r=2\left[\frac{1}{2}\left(c^{\prime}(D)-c\right)\right]$. Поэтому из теоремы Безу получаем нужную оценку, а экстремальное свойство является его прямым следствием.

Если объединение таких гнезд не исчерпьвает всю кривую, то оценку можно немного уточнить, взяв дополнительно $c^{\prime}(D)-c$ точек на овале, не входящем в гнезда.

4.6.11. ПРЕДЛОЖЕНИЕ. Пусть вещественная кривая $C$ имеет $s=\frac{1}{2} D$ 。 $(D-K)-l$ гнезд, лежащих вне друг друга. Eсли $s>\mu(l, D)$, то среди этих гнезд найдутся $s-\mu(l, D)+1$ гнезд, общее число овалов которых не больие, чем $\frac{1}{2} C \circ D-(\mu(l, D)-1)$.

ДокАЗАТЕЛЬСТВо. В каждом гнезде возьмем точку на самом внутреннем овале и рассмотрим линейную систему кривых в $|D|$, проходяших через эти точки. Ее размерность не меньше $l$, и по определению числа $\mu(l, D)$ она содержит кривую $D_{0}$ с числом компонент связности $\leqslant \mu(l, D)$. Поэтому в пересечение с $D_{0}$ не менее $s-\mu(l, D)+1$ гнезд дают вклад, не меньший их удвоенной глубины. Вклад каждого из остальных гнезд не меньше 2 благодаря их самым внутренним овалам. Остается применить неравенство Безу.

4.6.12. СлЕДСТВИЕ. Если кривая $C$ имеет $\frac{1}{2} D \circ(D-K)$ гнезд, лежащих вне друг друга, то среди этих гнезд найдутся $r=-D \circ K-1$ гнезд, общее число овалов которых не превосходит $\frac{1}{2} C \circ D-\frac{1}{2} D \circ(D+K)-1$.

ДоКАЗАТЕЛЬСТвО. Это следствие - специальный случай предложения 4.6.11, когда $l=0$. Действительно, $\mu(0, q)=\frac{1}{2} D \circ(D+K)+2$ и $r=\frac{1}{2} D \circ(D-K)-\mu(0, D)+1$.

4.7. Плоские кривые, проходящие через данные точки. В случае плоских кривых линейная система $|D|$ определяется степенью $q$ дивизора $D$. Поэтому речь идет просто о двух последовательностях $\{c(q)\},\left\{c^{\prime}(q)\right\}$. Их первые четыре члена известны.

\subsection{1. ПРЕДЛОЖЕНИЕ. Справедливы равенства}

$$
c(1)=c^{\prime}(1)=2, \quad c(2)=c^{\prime}(2)=5, \quad c(3)=c^{\prime}(3)=8 \quad u \quad c(4)=c^{\prime}(4)=13 .
$$

Заметим, что любой вешественньй пучок кубик, в действительности, содержит вешественную кубику со связной вещественной частью. 
ДокАЗАТЕльство. Множество вещественных точек любой вещественной кривой степени 1 или 2 связно. В силу 4.6.4 остается доказать, что

$$
8 \leqslant c(3), c^{\prime}(3)<9, \quad 13 \leqslant c(4), c^{\prime}(4)<14
$$

Неравенство $c(3) \geqslant 8$ следует из 4.7.3 ниже. Для доказательства неравенства $c^{\prime}(3)<9$ возьмем неприводимую вещественную кривую $A$ степени 3 с двумя вещественными компонентами, выберем 5 точек на ее двусторонней компоненте и 4 точки на односторонней. В силу двойственности Пуанкаре любая вешественная кривая $A^{\prime}$ степени 3 , проходящая через 9 выбранных точек, имеет 5 точек пересечения с односторонней компонентой (либо одна из четырех выбранных точек получается кратной). Тогда по теореме Безу $A^{\prime}$ совпадает с $A$, но ни одна из компонент кривой $A$ не содержит все 9 точек.

Неравенства $c(4) \geqslant 13$ является простым следствием п. 4.6.7.

Для доказательства неравенства $c^{\prime}(4)<14$ возьмем вешественную кривую $A$ степени 4 с 4 вешественными компонентами, выберем 11 точек на одной из них и по одной точке на каждом из остальных овалов. В силу двойственности Пуанкаре любая вещественная кривая $A^{\prime}$ степени 4, проходящая через 14 выбранных точек, пересекает каждый овал кривой $A$ еще по крайней мере в одной точке (или имеет кратную точку пересечения). Поэтому общее число точек пересечения $>16$, и по теореме Безу $A^{\prime}$ совпадает с $A$ (как кривая степени 4 с максимальным числом компонент $A$ неособа и неприводима). Остается заметить, что ни одна из компонент кривой $A^{\prime}=A$ не содержит все 14 точек.

ЗАмечАниЕ. В доказательстве неравенства $c_{3}^{\prime}<9$, приведенном вьше, 9 точек выбраны так, что они не лежат ни на одной вешественной кубике, отличной от $A$. Из доказательства следует также, что связную вещественную кубику можно провести через 9 точек $M$-кубики $C$ тогда и только тогда, когда эти точки лежат на кубике $C^{\prime}$, отличной от $C$. Сушествование кубики $C^{\prime}$ можно выразить в терминах групповой структуры на $C$ : она сушествует тогда и только тогда, когда сумма этих 9 точек равна 0 (или, точнее, 9-кратной точке перегиба). Конечно, множество из 9 точек должно быть инвариантным относительно комплексного сопряжения; тогда $C^{\prime}$ можно выбрать вешественной. Как следствие получаем, что девятки точек, не лежащих на связной кубике, образуют открытое непустое подмножество в пространстве всех девяток точек на $\mathbb{R} \mathbb{P}^{2}$.

Точное значение остальных членов последовательностей $\{c(q)\},\left\{c^{\prime}(q)\right\}$ неизвестно. Ниже мы даем несколько простых общих нижних и верхних границ.

4.7.2. ПРЕДЛОЖЕНИЕ. Для любого q справедливо неравенство $c(q) \geqslant 3 q-1$.

Отступление: вешественные рациональные кривые. Насколько нам известно, следуюший вопрос пока остается открытым: возможно ли провести неприводимую вещественную рациональную кривую (или, точнее, ее компоненту связности) степени q через любое множество $3 q-1$ вещественных точек в общем положении? В [99] на этот вопрос был дан утвердительный ответ, однако доказательство не было опубликовано; возможно, оно содержало пробел. С другой стороны, можно отбросить условие общего положения и поставить вопрос о существовании некоторой (не обязательно неприводимой) рациональной кривой. 
Первый нетривиальный случай (и единственный, где известен полный ответ) - когда $q=3$ : через 8 точек в общем положении можно провести 12 (рациональных) кубик; в зависимости от расположения точек, 8, 10 или 12 из них вещественны. (Все три значения реализуются; все 12 рациональных кубик в пучке вещественны тогда и только тогда, когда пучок содержит две кубики с уединенной вещественной двойной точкой.)

Имеется также несколько простых результатов о наборах точек с предписанными кратностями. Например, если $q-1$ из $3 q-1$ точек совпадают, ответ утвердительный. По сути дела, в аффинной системе координат, в которой кратная точка $M$ и одна из остальных $2 q$ точек, обозначенная через $N$, лежат на бесконечности, задача становится линейной: если ось $x$ провести через $N$, а ось $y$ - через $M$, то все сведется к интерполяции $2 q-1$ значений $y_{j}$ рациональной функцией $y=P(x) / Q(x)$ мультистепени $(\operatorname{deg} P, \operatorname{deg} Q)=(q-1, q-1)$. Явное решение этой задачи называется интерполяиионной формулой Коши. Как показывает это построение, через любые $2 q$ вешественных точек можно провести вещественную рациональную кривую степени $q$. Этот результат можно также получить соответствующим малым возмущением семейства $q$ прямых, проходящих через данные точки, рассматривая в пространстве $\mathbb{R} C_{q}$ вешественных кривых степени $q$ подмногообразие кривых, имеющих $\frac{1}{2}(q-1)(q-2)$ двойных точек. Замена прямых кониками или рациональньми кубиками дает несколько лучшие результаты: вешественную рациональную кривую степени $q$ можно провести через любые $\frac{5}{2} q$ или соответственно $\frac{8}{3} q$ вешественных точек.

4.7.3. ПрЕДЛОЖЕНИЕ. Для любих 8 точек в общем положении в $\mathbb{R P}^{2}$ обозначим через $c_{+}$(соответственно $c_{-}$) число вещественных кубик, проходящих через эти точки и имеющих уединенную (соответственно неуединенную) двойную точку. Тогда $c_{-}-c_{+}=8 u c_{-}+c_{+} \leqslant 12$. В частности, существует не менее 8 вещественных рациональных кубик, проходящих через данные точки.

ДокАЗАТЕльство. Пучок кубик, проходящих через 8 данных точек, определяет (особое) расслоение $X \rightarrow \mathbb{R P}^{1}$, где $X$ есть $\mathbb{R P}^{2}$, на которой эти 8 точек раздуты. Утверждение следует из вычисления эйлеровой характеристики пространства $X$.

Заметим, что аналогично доказывается утверждение: любой пучок кубик содержит кубику со связной вещественной частью.

ДОКАЗАТЕЛЬСТВО ПРЕДЛОЖЕНИЯ 4.7.2. В силу п. 4.7.1 достаточно рассмотреть случай $q>4$. Доказательство зависит от значения $q \bmod 4$.

Если $q=4 k+1, k \in \mathbb{Z}$, то $3(4 k+1)-1=12 k+2$. Проведем вешественную прямую через любые две из $12 k+2$ точек. Остальные $12 k$ точек разобьем на $k$ групп по 12 точек. Каждую группу и одну из первых двух точек можно соединить вешественной кривой степени 4 со связным множеством вещественных точек. Объединение указанной прямой и построенных кривых степени 4 имеет степень $4 k+1$; его множество вешественных точек связно и содержит все $12 k+2$ точек.

Если $q=4 k+2, k \in \mathbb{Z}$, то $3(4 k+2)-1=12 k+5$. Проведем вешественную конику через любые 5 из $12 k+5$ точек. Остальные $12 k$ точек разобьем на $k$ групп по 12 точек. Каждую группу и одну из первых пяти точек можно соединить вещественной кривой степени 4 со связным множеством вещественных точек. Объединение коники и построенных кривых степени 4 имеет степень $4 k+2$; его вещественная часть связна и содержит все $12 k+5$ точек. 
Если $q=4 k+3, k \in \mathbb{Z}$, то $3(4 k+3)-1=12 k+8$, и кривую можно построить, как и вьше, начиная с кубики, проходяшей через любые 8 из $12 k+8$ точек.

Если $q=4 k, k \in \mathbb{Z}$, то границу $12 k-1$ можно заменить на $12 k+1$. Выберем одну из этих точек. Остальные $12 k$ точек разобьем на $k$ групп по 12 точек. Каждую группу и выбранную точку можно соединить вешественной кривой степени 4 со связным множеством вещественных точек. Объединение этих кривых имеет степень $4 k$, множество его вещественных точек связно и содержит все $12 k+1$ точек.

4.7.4. ПРЕДЛОЖЕНИЕ. Если $q$ нечетно, оо $^{\prime}(q) \leqslant \frac{1}{2} q(q+3)-\left[\frac{1}{8}\left(q^{2}-1\right)\right]$. Если $q$ четно, то $c^{\prime}(q) \leqslant \frac{1}{2} q(q+3)-\left[\frac{1}{8} q(q-2)\right]$.

ДокаЗАтЕльство. Сначала рассмотрим случай нечетного $q \geqslant 3$. (Для $q=1$ неравенство следует из $c^{\prime}(1)=2$.) Положим $q=2 k-1$ и $m=k+1$. Тогда граница примет вид $c^{\prime}(q) \leqslant \frac{3}{2} k(k+1)-1$. Возьмем $M$-кривую $A$ степени $m$. Ее вещественная часть имеет $\frac{1}{2} k(k-1)+1$ компонент связности. Выберем $3 k$ точек на одной из компонент и по 3 точки на каждой из остальных - всего $\frac{3}{2} k(k+1)$ точек. Если $k$ четно, выбранная компонента должна быть односторонней. Пусть $c^{\prime}(q) \geqslant \frac{3}{2} k(k+1)$. Тогда сушествует вешественная кривая $B$ степени $q$ с компонентой связности, содержашей все $\frac{3}{2} k(k+1)$ точек. В силу двойственности Пуанкаре каждая компонента множества $\mathbb{R} A$ пересекает $\mathbb{R} B$ по крайней мере в одной дополнительной точке (или одна из точек пересечения кратная). Поэтому число точек пересечения кривых $A$ и $B$ с учетом их кратностей не меньше, чем $2 k^{2}+k+1>m q$, и, значит, по теореме Безу кривая $B$ содержит $A$ в качестве компоненты (как $M$-кривая, $A$ неприводима). Следовательно, $B$ распадается на две кривые $A$ и $B^{\prime}$ степеней $m=k+1$ и $q-m=k-2$ соответственно. Более того, $\mathbb{R} A$ содержится в компоненте связности множества $\mathbb{R} B^{\prime}$; таким образом, $A$ и $B^{\prime}$ пересекаются не менее чем в $k(k-1)>(k+1)(k-2)$ точках. Поскольку $A$ неприводима, это противоречит теореме Безу.

Если $q$ четно, положим $q=2 k$ и $m=q+4=2(k+2)$ и рассмотрим $M$-кривую Вимана $A$ степени $m$ (т.е. кривую с вешественной схемой $\langle(k+1)(k+3)+1\rangle \sqcup$ $\frac{1}{2} k(k+1)\langle 1\langle 1\rangle\rangle$; ее можно построить малым возмущением квадрата $M$-кривой степени $k+2)$. Пусть $c^{\prime}(q) \geqslant \frac{1}{2} q(q+3)-\frac{1}{8} q(q-2)+1=\frac{3}{2} k(k+1)+2 k+1$. Выберем по одной точке на внутреннем овале каждого гнезда кривой $A$ и по одной точке в $k^{2}+3 k+1$ из $k^{2}+4 k+4$ пустых внешних овалов- всего $\frac{3}{2} k(k+1)+2 k+1$ точек, и проведем через них компоненту связности вещественной кривой $B$ степени $q$. Тогда $A$ и $B$ пересекаются не менее чем в $4 k^{2}+8 k+2>4 k^{2}+8 k$ точках, что противоречит теореме Безу.

ЗАмЕчАниЕ. В случае нечетного $q$ можно дать иное доказательство, аналогичное случаю четного $q$. Удивительно, но это не улучшает оценку.

4.7.6. СлЕДСТВИЕ. Справедливы неравенства

$$
14 \leqslant c(5) \leqslant c^{\prime}(5) \leqslant 17, \quad 17 \leqslant c(6) \leqslant c^{\prime}(6) \leqslant 24 \quad u \quad 20 \leqslant c(7) \leqslant c^{\prime}(7) \leqslant 29 .
$$

Верхняя граница для $c^{\prime}(6)$ легко улучшается:

4.7.6. ПРЕДЛОЖЕНИЕ. Справедливо неравенство $c^{\prime}(6) \leqslant 23$. 
ДокАЗАТЕЛЬСТвО. Пусть $c^{\prime}(6) \geqslant 24$. Возьмем (очевидно, неприводимую) $M$-кривую степени 9 со схемой $J \sqcup\langle 1\langle a\rangle\rangle \sqcup\langle 1\langle b\rangle\rangle \sqcup\langle 1\langle c\rangle\rangle \sqcup\langle 1\langle d\rangle\rangle, a, b, c, d>0, a+b+c+d=24$, и проведем компоненту связности вещественной секстики через все 24 пустых овала. Эти две кривые пересекаются не менее чем в $48+8=56>54$ точках.

4.8. Препятствия к жесткой изотопии. Топологические характеристики вешественной кривой такие, как ее вешественная схема, тип, комплексная схема (т.е. вешественная схема, обогашенная комплексньми ориентациями) для кривой типа I, сохраняются при жестких изотопиях. Другим мошньм источником препятствий является теорема Безу, примененная к соответствуюшим вспомогательным кривым.

Насколько нам известно, препятствия, полученные из теоремы Безу, никогда не изучались систематически. Поэтому мы ограничимся объяснением основной идеи и ее простейшими реализациями, взятыми из [34] и [77].

Начнем со вспомогательных кривых наименшей степени: рассмотрим прямые. В этом случае мы ишем пары точек, для простоты - в $\mathbb{R} \mathbb{P}^{2} \backslash \mathbb{R} A$, расположенных топологически таким образом, чтобы прямая $L$, проходящая через них, пересекала кривую $A$ только в вещественных точках. Если такой выбор возможен, положение множества $\mathbb{R} A$ относительно $\mathbb{R} L$ сохраняется при жестких изотопиях кривой $A$. В частности, множество $\mathbb{R} A$ расположено одинаково относительно всех вешественных прямых, близких к $L$.

Чтобы сделать эту идею более конструктивной, введем следующее определение. Вешественная прямая $L$ с двумя отмеченньми точками $p_{1}, p_{2} \in \mathbb{R} L$, не лежашими на вешественной части неособой вешественной кривой $A$, называется замком кривой $A$, если (1) число точек в $S=\mathbb{R} L \cap \mathbb{R} A$ равно степени кривой $A,(2)$ для любой пары (возможно совпадающих) точек из $S$, ограничивающих интервал в $\mathbb{R} L \backslash\left(\left\{p_{1}, p_{2}\right\} \cup S\right)$ и дугу в $\mathbb{R} A \backslash S$, цикл, образованньй этими сегментом и дугой, либо не гомологичен нулю в $\mathbb{R} \mathbb{P}^{2}$, либо охватьвает хотя бы один овал кривой $A$. Ясно, что замок непрерывно продолжается на любую жесткую изотопию кривой $A$ и, более того, независимо от продолжения топологический тип четверки $\left(\mathbb{R}^{2}, \mathbb{R} A \cup \mathbb{R} L, \mathbb{R} L,\left\{p_{1}, p_{2}\right\}\right)$ не меняется. Даже это простое рассмотрение дает нам два инварианта: существование замка и топологические типы четверок, соответствуюших всем замкам.

В качестве примера рассмотрим две кривые степени 6 со схемой $\langle 2\rangle \sqcup\langle 1\langle 2\rangle\rangle$, построенные из двух объединений эллипсов так, как показано на рис. 2 . У первой кривой нет замков. Для второй - любая пара точек, взятых по одной внутри каждого из двух внешних овалов, определяет замок. Значит, эти кривые не жестко изотопны.
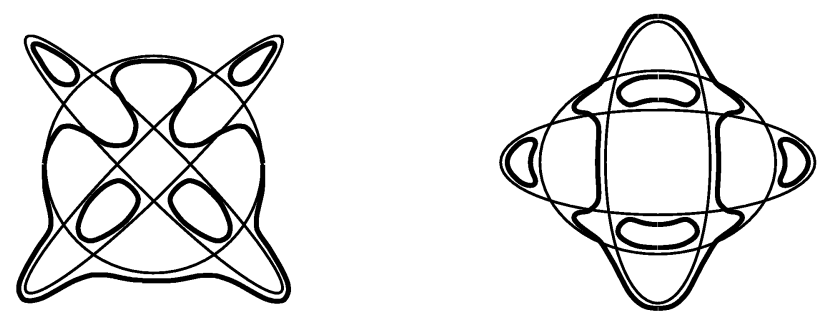

Рис. 2. Секстики без замка и с замком

Бывают ситуации, когда полезно рассматривать одновременно несколько замков. Пример - две кривые степени 5 с 4 овалами, показанные на рис. 3. Для обеих кривых 
любая пара овалов определяет замок; поэтому любая тройка определяет тройной замок. Первая кривая имеет тройку овалов, тройной замок которой обладает следующим свойством: одна из компонент связности дополнения трех прямых замка содержит четвертый овал и не пересекает одностороннюю компоненту. Другая кривая не имеет такой тройки овалов. Значит, эти кривые не являются жестко изотопными.
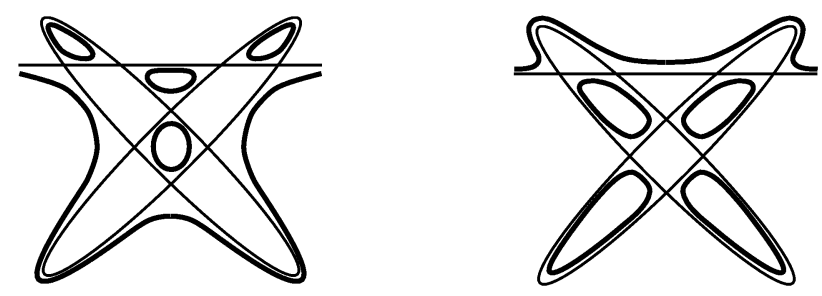

Рис. 3. Две квинтики с различными тройными замками

Фактически, в обоих примерах кривые отличаются своей комплексной схемой: как видно из построения, в каждом примере одна кривая принадлежит типу I, а другая - типу II. Пары не жестко изотопных кривых с одной и той же комплексной схемой впервые появляются в степени 7. Пример, принадлежаший А. Марену [77], показан на рис. 4. Две кривые с комплексной схемой $\left\langle J \sqcup 1^{+}\left\langle 1^{+} \sqcup 2^{-}\right\rangle \sqcup 6^{+} \sqcup 5^{-}\right\rangle$строятся методом Гильберта с различием в построениях на последнем шаге. Три внутренних овала определяют тройной замок, и указанные кривые отличаются распределением внешних овалов между компонентами связности дополнения трех прямых.
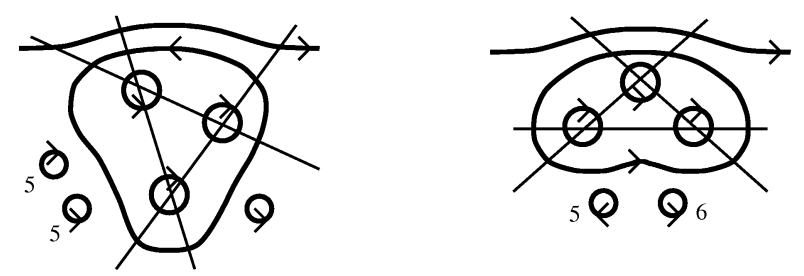

Рис. 4. Две кривые степени 7 с различными тройными замками

Другой пример не жестко изотопных кривых степени 7 с одной и той же комплексной схемой был построен Т. Фидлером [34]. (В его примере кривые неразбиваюшие.)

Сушествуют примеры, где в качестве замков вместо прямых используются коники. В одном из них, принадлежашем И.В. Итенбергу, внутренние овалы двух отрицательных инъективных пар секстики $\left\langle 1\left\langle 3^{+} \sqcup 2^{-}\right\rangle\right\rangle \sqcup\langle 5\rangle$ отделяются друг от друга коникой, пересекаюшей три остальных внутренних овала и два внешних. Можно, конечно, рассматривать и замки большей степени. Мы заканчиваем обсуждение этой темы, поскольку она еше не достаточно понята.

Возврашаясь к примеру Марена, заметим, что неизвестно, являются ли эти кривые эквивариантно изотопными. Более общо, две следующих фундаментальных проблемы до сих пор открыты: Существуют ли препятствия, связанные с теоремой Безу и не являющиеся гибкими? Существуют ли эквивариантно изотопные, но не жестко изотопные кривые? (Когда этот обзор был готов, С. Фидлер-Ле Тузе и 
С. Ю. Оревков сообшили, что они нашли гибкую аффинную секстику, не реализуемую алгебраически вследствие теоремы Безу, примененной к пересечениям с кубиками.)

4.9. Кривые на квадриках. Мы заключаем этот раздел кратким перечислением известных результатов, относяшихся к кривым на неособых квадриках. Это, по-видимому, единственньй (кроме $\mathbb{P}^{2}$ ) серьезно изучавшийся случай.

Пусть $X$ - неособая квадрика. С комплексной точки зрения $X=\mathbb{P}^{1} \times \mathbb{P}^{1}$, и на $X$ можно ввести две, с точностью до биголоморфизма, антиголоморфные инволюции с непустой вещественной частью; в результате получаются две вешественные поверхности: гиперболоид, являющийся $M$-поверхностью с вещественной частью, гомеоморфной тору, и эллипсоид - $(M-1)$-поверхность с $\mathbb{R} X \cong S^{2}$.

Зафиксируем пару $P_{1}, P_{2}$ образуюших квадрики $X$. Фундаментальные классы $\left[P_{1}\right]$, $\left[P_{2}\right]$ образуют базис группы $H_{2}(\mathbb{C} X) \cong \mathbb{Z} \oplus \mathbb{Z}$; форма пересечений задается равенствами $\left[P_{1}\right]^{2}=\left[P_{2}\right]^{2}=0,\left[P_{1}\right] \circ\left[P_{2}\right]=1$. Пусть $A$ - алгебраическая кривая на $X$. Тогда $[A]=m_{1}\left[P_{1}\right]+m_{2}\left[P_{2}\right]$ для некоторых неотрицательных целых $m_{1}, m_{2}$. Пара $\left(m_{1}, m_{2}\right)$ назьвается бистепенъю кривой $A$. Если $\left[x_{0}: x_{1}\right]$, [y $\left.: y_{1}\right]$ - однородные координаты на прямых $P_{1}, P_{2}$, то кривая $A$ задается биоднородным многочленом

$$
F\left(x_{0}, x_{1} ; y_{0}, y_{1}\right)=\sum_{i, j=1}^{m_{1}, m_{2}} a_{i j} x_{1}^{i} x_{0}^{m_{1}-i} y_{1}^{j} y_{0}^{m_{2}-j}
$$

имеющим степени однородности $m_{1}$ по $x_{0}, x_{1}$ и $m_{2}$ по $y_{0}, y_{1}$. Такие кривые образуют проективное пространство размерности $m_{1} m_{2}+m_{1}+m_{2}$; если $A$ неособа, ее род равен $\left(m_{1}-1\right)\left(m_{2}-1\right)\left(\right.$ см. $(4.3 .1) ; K_{X}$ имеет бистепень $\left.(-2,-2)\right)$.

На гиперболоиде антиголоморфная инволюция соnј переводит каждую из четырех координат в комплексно сопряженную; поэтому кривая $A$ вешественна тогда и только тогда, когда все $a_{i j}$ вешественны. На эллипсоиде инволюция conj действует по формуле $\left(\left[x_{0}: x_{1}\right],\left[y_{0}: y_{1}\right]\right) \mapsto\left(\left[\bar{y}_{0}: \bar{y}_{1}\right],\left[\bar{x}_{0}: \bar{x}_{1}\right]\right)$, и вешественность кривой $A$ означает, что $a_{i j}=\bar{a}_{j i}$ (в частности, $m_{1}=m_{2}$ ).

Далее кривая $A$ всюду считается вещественной. Пусть сначала $(X$, conj) - гиперболоид. Фундаментальные классы $\left[\mathbb{R} P_{1}\right],\left[\mathbb{R} P_{2}\right]$, наделенные некоторыми (фиксированными) ориентациями, образуют базис группы $H_{1}(\mathbb{R} X) \cong \mathbb{Z} \oplus \mathbb{Z}$. Вещественная часть $\mathbb{R} A$ может иметь компоненты двух типов: стягиваемые в $\mathbb{R} X$ и нестягиваемые; стягиваемые компоненты назьваются овалами. Число овалов обозначается через $l$, число нестягиваемых компонент - через $h$. Каждый овал ограничивает топологический диск в $\mathbb{R} X$, назьваемый внутренностью овала. Все нестягиваемые компоненты $N_{1}, \ldots, N_{h}$ реализуют один и тот же ненулевой класс $\left(c_{1}, c_{2}\right)$ в $H_{1}(\mathbb{R} X)$, где $c_{1}, c_{2}$ взаимно просты. Вешественная схема кривой $\mathbb{R} A \subset \mathbb{R} X$ кодируется следуюшим образом:

$$
\left\langle\left(c_{1}, c_{2}\right), \text { scheme }_{1},\left(c_{1}, c_{2}\right), \text { scheme }_{2}, \ldots,\left(c_{1}, c_{2}\right), \text { scheme }_{h}\right\rangle
$$

где scheme ${ }_{1}, \ldots$, scheme $_{h}$ - схемы расположения овалов, лежащих в компонентах связности поверхности $\mathbb{R} X \backslash\left(N_{1} \cup \cdots \cup N_{h}\right)$.

Если оба числа $m_{1}$ и $m_{2}$ четны, $\mathbb{R} A$ разделяет $\mathbb{R} X$ на две части $\mathbb{R} X_{ \pm}$. Ясно, что $\chi\left(\mathbb{R} X_{-}\right)=-\chi\left(\mathbb{R} X_{+}\right)$. Если все компоненты множества $\mathbb{R} A$ являются овалами, будем считать, что $\mathbb{R} X_{+}-$поверхность рода 1 . 
4.9.1. ЗАПРЕТЫ ДЛЯ КРИВЫХ НА ГИПЕРБОЛОИДЕ. Пусть $A-$ вещественная кривая бистепени $\left(m_{1}, m_{2}\right)$ на гиперболоиде $X$. Тогда определенные выше $l, h$, $c_{1}, c_{2}$ удовлетворяют следующим ограничениям.

Неравенство Харнака:

(1) $l+h \leqslant\left(m_{1}-1\right)\left(m_{2}-1\right)+1$.

Сравнения. Пусть $m_{1}, m_{2}$ четны $u \frac{1}{2}\left(m_{1} c_{2}+m_{2} c_{1}\right)=c_{1} c_{2} \bmod 2$. Тогда $(n p u$ сделанном соглашении о въборе $\mathbb{R} X_{+}$)

(2) если $A$ есть $M$-кривая, то $\chi\left(\mathbb{R} X_{+}\right)=\frac{1}{2} m_{1} m_{2} \bmod 8$;

(3) если $A$ есть $(M-1)$-кривая, то $\chi\left(\mathbb{R} X_{+}\right)=\frac{1}{2} m_{1} m_{2} \pm 1 \bmod 8$;

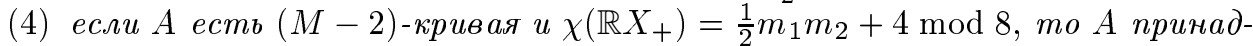
лехит muny I;

(5) если А принадлежст типу I, то $\chi\left(\mathbb{R} X_{+}\right)=0 \bmod 4$.

Следствия теоремы Безу:

(6) $h c_{i}=m_{1} \bmod 2 u h\left|c_{i}\right| \leqslant m_{1}$ для $i=1,2$;

(7) если $\mathbb{R} A$ имеет $r$ попарно дизбюнктных гнезд глубины $s_{1}, \ldots, s_{r}$, то справедливо неравенство

$$
\sum_{j=1}^{r} s_{j} \leqslant \frac{1}{2}\left(m_{1}-h\left|c_{1}\right|+[r / 2]\left(m_{2}+h\left|c_{2}\right|\right)\right)
$$

( и такое жее неравенство, полученное заменой $m_{1}, c_{1}$ на $m_{2}, c_{2}$ ).

ДокАЗАтЕльство. Утверждение (1) есть классическое неравенство ХарнакаКлейна. Утверждения (2)-(5) следуют из 3.3.2 и 3.3.4. Утверждение (6) есть вешественньй вариант теоремы Безу (см. 4.6), примененный к $A$ и кривым бистепени $(1,0)$ и $(0,1)$, а неравенство (7) доказывается применением теоремы Безу к $A$ и кривой бистепени $([r / 2], 1)$, проведенной через $r$ точек, взятых внутри самых внутренних овалов гнезд (см. [130]).

ЗАмЕчАниЕ. В более слабой форме указанные сравнения появились в [79], [80], что побудило Михалкина [81], [83] улучшить и обобшить их до теоремы 3.3.2.

Если $(X$, conj) - эллипсоид, то все компоненты кривой $\mathbb{R} A$ стягиваемы и называются овалами; их число обозначается через $l$. В этом случае мы фиксируем точку $\infty \in \mathbb{R} X \backslash \mathbb{R} A$, назьваемую внешней, и для овала $C \subset \mathbb{R} X$ определяем его внутренность как компоненту поверхности $\mathbb{R} X \backslash C$, не содержащую $\infty$. При этом на множестве овалов возникает естественный частичный порядок, и схема расположения овалов кодируется как обычно.

Поскольку $\mathbb{R} X \cong S^{2}$, любая кривая $\mathbb{R} A$ разделяет $\mathbb{R} X$ на две части, обозначаемые через $\mathbb{R} X_{ \pm}$.

4.9.2. ЗАПРЕТЫ ДЛЯ КРИВЫХ НА ЭЛЛИПСОИДЕ. Пусть $A$ - вещественная кривая бистепени $(m, m)$ на әллипсоиде $X$ и $l$ - число компонент множества $\mathbb{R} A$. Тогда А удовлетворяет следующим ограничениям.

Неравенство Харнака:

(1) $l \leqslant\left(m_{1}-1\right)\left(m_{2}-1\right)+1$. 
Сравнения. Пусть $m$ нечетно.

(2) Если А есть $M$-кривая, то $\chi\left(\mathbb{R} X_{ \pm}\right)=\frac{1}{2}\left(m^{2}+1\right) \bmod 8$.

(3) Если $A$ есть $(M-1)$-кривая, то $\chi\left(\mathbb{R} X_{ \pm}\right)=\frac{1}{2}\left(m^{2}+1\right) \pm 1 \bmod 8$.

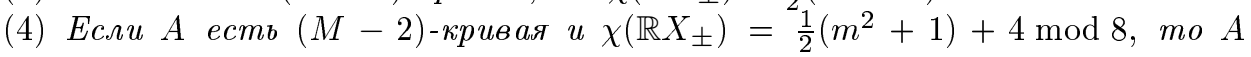
принадлежит типу I.

(5) Если А принадлежит типу I, mо $\chi\left(\mathbb{R} X_{ \pm}\right)=1 \bmod 4$.

Следствия теоремы Безу:

(6) Общее число овалов любых трех попарно дизбюнктных гнезд не превосxодит $m$.

Доказательство аналогично доказательству утверждения 4.9.1.

Два следующих хорошо известных утверждения можно найти, например, в [131]. Их стандартные доказательства, воспроизводимые ниже, служат моделью доказательств в ряде подобных случаев (таких, как рациональные и гиперэллиптические кривые на линейчатых поверхностях, пересечения квадрик или гомотопическая классификация невырожденных однородных полиномиальных векторных полей).

4.9.3. КРИВЫЕ БИСТЕПЕНИ $(m, 1)$. Кривая бистепени $(m, 1)$ на гиперболоиде определяется с точностью до жсесткой изотопии своей вещественной схемой: $\langle(w, 1)\rangle c-m \leqslant w \leqslant m u w=m \bmod 2$. Все такие кривые принадлежст типу I.

На әллипсоиде кривье бистепени $(1,1)$ образуют два экестких изотопических класса: $\langle 0\rangle$ и $\langle 1\rangle$, последний состоит из кривых типа I.

ДокАЗАТЕльство. Неособая кривая бистепени $(m, 1)$ на гиперболоиде задается уравнением

$$
y_{0} a\left(x_{0}, x_{1}\right)+y_{1} b\left(x_{0}, x_{1}\right)=0,
$$

где $a$ и $b$ - ненулевые однородные многочлены степени $m$ без общих корней. Многочлены $a$ и $b$ своими корнями определяются с точностью до постоянного множителя. Любые два соседних вещественных корня одного из этих многочленов, не разделенные вещественными корнями другого, можно удалить в мнимую область. После удаления всех таких пар $|w|$ есть число вещественных корней многочлена $a$ (или $b$ ); каждому ненулевому значению $|w|$ отвечают два класса кривых, отличающихся знаками многочленов $a$ и $b$ в фиксированной обшей точке.

Любая кривая бистепени $(1,1)$ на эллипсоиде высекается плоскостью, поэтому, очевидно, возможны лишш два указанных случая.

4.9.4. КРИВЫЕ БИСТЕПЕНИ $(m, 2)$. Кривая бистепени $(m, 2)$ на әиперболоиде определяется с точностью до жесткой изотопии своей вещественной схемой, имеющей вид

$$
\left\langle(0,1), \alpha_{1},(0,1), \alpha_{2}, \ldots,(0,1), \alpha_{h}\right\rangle,
$$

əде $0 \leqslant h \leqslant m, h=m \bmod 2 u h+\sum \alpha_{i} \leqslant m$, либо

$$
\left\langle 2\left(\frac{1}{2} w, 1\right)\right\rangle \text { при четном } m u\langle(w, 2)\rangle \text { при нечетном } m,
$$

где $-m \leqslant w \leqslant m u w=m \bmod 2$. Последняя схема (и при четном, и при нечетном $(m)$ и $М$-схемь принадлежсат типу I; остальные схемь - типу II. 
На эллипсоиде кривые бистепени $(2,2)$ образуют три жестких изотопических класса: $\varnothing,\langle 1\rangle$ u $\langle 2\rangle$. Тип I имеет лишь схема $\langle 2\rangle$.

Приведенное ниже доказательство справедливо более или менее без изменений во всех случаях, когда рассматриваемые кривые гиперэллиптичны (и гиперэллиптическая структура индуцируется расслоением поверхности на прямые). По этой причине мы воспроизводим все сушественные детали.

ДокАЗАТЕльство.Кривая бистепени $(m, 2)$ на гиперболоиде задается уравнени$\mathrm{em}$

$$
y_{0}^{2} a\left(x_{0}, x_{1}\right)+2 y_{0} y_{1} b\left(x_{0}, x_{1}\right)+y_{1}^{2} c\left(x_{0}, x_{1}\right)=0,
$$

где $a, b$ и $c$ - однородные многочлены степени $m$. Такая кривая неособа тогда и только тогда, когда дискриминант $D=b^{2}-a c$ не имеет кратных корней.

Положим $V=\left\{\left(x_{0}: x_{1}\right) \in \mathbb{R P}^{1} \mid D\left(x_{0}, x_{1}\right) \geqslant 0\right\}$. Рассмотрим аффинную часть $x_{1} \neq 0$. Компоненты связности множества, состояшего из неособых кривых с фиксированным $V$ и фиксированными корнями $u_{1}, \ldots, u_{m}$ многочлена $a$ (которые могут быть кратными), определяются выбором значений $b\left(u_{i}\right)= \pm \sqrt{D\left(u_{i}\right)}, i=1, \ldots, m$, т.е. выбором знака многочлена $b$ в вешественных корнях многочлена $a$ (которые, очевидно, принадлежат $V$ ) и одной из двух ветвей квадратного корня в каждой паре комплексно сопряженных корней. Эти компоненты можно соединить, используя следующие движения:

(1) передвижение в мнимую область пары соседних вешественных корней многочлена $a$, в которых $b$ имеет один и тот же знак; и наоборот, передвижение пары комплексно сопряженных корней в произвольную компоненту множества $V$;

(2) изменение знака многочлена $b$ в вещественном корне многочлена $a$, не отделенном от $\partial V$ другими корнями, передвижением этого корня до $\partial V$ и обратно;

(3) замена ветви $\sqrt{D}$ на противоположную в любой паре комплексно сопряженных корней многочлена $a$ передвижением этой пары сквозь пару корней (возможно совпадающих) дискриминанта $D$.

Следовательно, если $V=\varnothing$ или $\partial V \neq \varnothing$, знак многочлена $b$ в каждом (вешественном или мнимом) корне многочлена $a$ можно обратить; поэтому кривая в этом случае определяется с точностью до жесткой изотопии компонентами множества $V$ и вычетом по модулю 2 числа вешественных корней многочлена $a$ в каждой такой компоненте: соответствующая компонента кривой является овалом, если это число четно, или компонентой класса $(1,0)$, если оно нечетно. Если множество $V$ совпадает с $\mathbb{R P}^{1}$, то оно содержит $|w|$ вешественных корней многочлена $a$, причем многочлен $b$ имеет противоположные знаки в любых двух соседних корнях; это дает вторую группу вешественных схем в 4.9.4.

Рассмотрим теперь кривые на эллипсоиде. Все пустые кривые жестко изотопны (на самом деле для любой бистепени), так как они образуют выпуклое множество. Если кривая непуста, раздуем на ней вешественную точку и стянем собственные прообразы двух мнимых образующих, проходящих через эту точку. В результате получим неособую кубику в $\mathbb{R} \mathbb{P}^{2}$. Обратное преобразование задается парой комплексно сопряженных точек этой кубики. Два жестких изотопических класса кубик соответствуют двум указанным в формулировке классам кривых на эллипсоиде. 
4.9.5. КРИВЫЕ БИСТЕПЕНИ $(3,3)$. Кривая бистепени $(3,3)$ на гиперболоиде или әллипсоиде с точностью до жесткой изотопии определяется своей вещественной схемой и, в случае гиперболоида, своим типом. Кривъе на гиперболоиде имеют следующие вещественнье схеми:

$$
\langle(1, \pm 1), \alpha\rangle \quad c \quad \alpha \leqslant 4, \quad\langle 3(1, \pm 1)\rangle, \quad\langle(3, \pm 1)\rangle, \quad\langle(1, \pm 3)\rangle .
$$

Схемь $\langle(1, \pm 1), 4\rangle,\langle 3(1, \pm 1)\rangle,\langle(3, \pm 1)\rangle$ u $\langle(1, \pm 3)\rangle$ принадлежсат типу $\mathrm{I} ;$ схема $\langle(1, \pm 1), 2\rangle$ - неопределенному типу; остальные схемы - типу II. Кривье на әллипсоиде имеют следующие вещественные схемы:

$$
\langle\alpha\rangle \quad c \quad \alpha \leqslant 5 \quad u \quad\langle 1\langle 1\langle 1\rangle\rangle\rangle \text {. }
$$

Схемь $\langle 5\rangle$ и $\langle 1\langle 1\langle 1\rangle\rangle\rangle$ принадлежсат типу I; остальные - типу II.

Как и в 3.5.2, доказательство утверждения 4.9.5 основано на перечислении стенок в пространстве кривых; последняя проблема, в основном, сводится к классификации плоских квартик с фиксированной касательной. Подробности - в [28].

В остальных пунктах этого раздела (предложения 4.9.6-4.9.8) даются только списки возможных схем для определенных бистепеней. Все схемы вне списков запрещаются п. 4.9.1 и 4.9.2. Указанные в списках схемы реализованы Д. А. Гудковым, А. К. Усачевым и Е.И. Шустиным (см. [42], [44] и [43]). У тверждения о типах схем следуют, в основном, из формулы Рохлина для комплексных ориентаций 4.2.2. Заметим также, что рассматриваемые кривые имеют те и только те ориентации, которые не противоречат формулам Рохлина и Оревкова. Подробности и реализация схем неопределенного типа имеются в работе Звонилова [130].

4.9.6. СХЕМЫ БИСТЕПЕНИ $(m, 3)$. Кривая бистепени $(m, 3)$ c $m>3$ на гиперболоиде может иметь только одну из следующих вещественных схем:

(1) $\langle 3(w, 1)\rangle$ c $3|w| \leqslant m u w=m \bmod 2$;

(2) $\langle(w, 3)\rangle c|w| \leqslant m, w=m \bmod 2 u w \neq 0 \bmod 3$;

(3) $\langle(m, \pm 1)\rangle$;

(4) $\langle(w, 1), \alpha\rangle c|w| \leqslant m-2, w=m \bmod 2 u 0 \leqslant \alpha \leqslant 2 m-2$.

Схемь (1)-(3) принадлежсат типу I; схемы (4) - типу I при $\alpha=2 m-2$ и типу II при а нечетном или равном 0 .

Все указанные схемы (вместе с их типами) реализованы А.Б. Корчагиным [69], которьй использовал метод склеивания Виро. Согласно В.И. Звонилову они могут быть реализованы также $T$-кривыми. Новый многообешающий подход к изучению тригональных кривых на линейчатых поверхностях, применяющий dessins d'enfant Гротендика к дискриминанту кривой, был недавно предложен С. Ю. Оревковым [93].

4.9.7. СХЕМЫ БИСТЕПЕНИ $(4,4)$ НА ГИПЕРБОЛОИДЕ. Следующий сnисок вещественных схем бистепени $(4,4)$ на гиперболоиде является полным:

(1) $\langle 4(1,0)\rangle,\langle 4(0,1)\rangle u\langle 4(1, \pm 1)\rangle$;

(2) $\langle 2(2, \pm 1)\rangle u\langle 2(1, \pm 2)\rangle$;

(3) $\langle(1, \pm 1), \alpha,(1, \pm 1), \beta\rangle c 0 \leqslant \alpha+\beta \leqslant 8 u \alpha \geqslant \beta$;

(4) $\langle(1,0), \alpha,(1,0), \beta\rangle u\langle(0,1), \alpha,(0,1), \beta\rangle$, где либо $0 \leqslant \alpha+\beta \leqslant 6 u \alpha \geqslant \beta$, либо $(\alpha, \beta)=(8,0),(4,4),(7,0)$ или $(4,3)$; 
(5) $\langle\alpha \sqcup 1\langle\beta\rangle\rangle$, əде либо $0 \leqslant \alpha+\beta \leqslant 7$, либо $(\alpha, \beta)=(0,9),(4,5),(8,1),(0,8)$, $(3,5),(4,4),(7,1)$ или $(8,0)$;

(6) $\langle 2\langle 1\rangle\rangle$

(7) $\langle 0\rangle$

Схемь (1), (2) и (6) принадлежат типу I. Схема (7) принадлежит типу II. Схемы (3) принадлежсат типу I, если $\alpha+\beta=8$, принадлежсат типу II, если $\alpha+\beta$ нечетно или $(\alpha, \beta)=(0,0),(1,1)$ или $(4,0)$, и принадлежат неопределенному типу в противном случае. Схемы (4) принадлежат типу I, если $(\alpha, \beta)=(8,0)$, $(4,4)$ или $(5,1)$, принадлежсат неопределенному типу, если $(\alpha, \beta)=(3,3),(4,0)$, $(2,2)$ или $(1,1)$, и принадлежат типу II в остальных случаях. Схемы (5) принадлежат типу $\mathrm{I}$, если $(\alpha, \beta)=(0,9),(4,5),(8,1),(1,6)$ или $(5,2)$, принадлежсат неопределенному типу, если $(\alpha, \beta)=(3,4),(7,0),(2,3)$ или $(4,1)$, и принадлежсат типу II в противном случае.

4.9.8. СХЕМЫ БИСТЕПЕНИ $(4,4)$ НА ЭЛЛИПСОИДЕ. Следующий список вещественных схем бистепени $(4,4)$ на әллипсоиде является полным:

(1) $\langle\alpha \sqcup 1\langle\beta\rangle\rangle c 0 \leqslant \alpha+\beta \leqslant 9 u \alpha \geqslant \beta$;

(2) $\langle 1\langle 1\langle 1\langle 1\rangle\rangle\rangle\rangle$;

(3) $\langle 0\rangle$.

Типу I принадлежсат схемь (2) и (1) при $\alpha+\beta=9$; типу II принадлежсат схемы (3) и (1), если $\alpha+\beta \leqslant 4$, или $\alpha+\beta$ четно, или $(\alpha, \beta)=(5,0)$; остальнье схемы принадлехсат неопределенному типу.

Двулистное накрывающее квадрики с ветвлением над кривой бистепени $(4,4)$ есть $K 3$-поверхность, группа Пикара которой содержит $U(2)$ (т.е. пару $a, b$ с $a^{2}=b^{2}=0$ и $a b=2)$, и обратно, любая $K 3$-поверхность $X$ с выделенной подгруппой $U(2) \subset$ Pic $X$ получается таким образом (см., например, [88]). Поэтому кривые бистепени $(4,4)$ можно изучать так же, как плоские секстики. В частности, с помощью этой техники можно получить их жесткую изотопическую классификацию. Первые шаги в этом направлении сделаны в [103]. В случае гиперболоида будет не удивительно, если список 4.9.7 совпадет с классификацией с точностью до жестких изотопий и автоморфизмов поверхности. (В случае эллипсоида возможно появление дополнительных инвариантов.) Заметим, что некоторые кривые на гиперболоиде, например, $\langle 1\langle 9\rangle\rangle$, $\langle 4 \sqcup 1\langle 5\rangle\rangle,\langle 1\langle 8\rangle\rangle,\langle 3 \sqcup 1\langle 5\rangle\rangle$ и $\langle 4 \sqcup 1\langle 4\rangle\rangle$, не являются киральными, см. [61].

О кривых на поверхностях большей степени известно мало. Можно лишш сослаться на [106] и [84], где на вешественных кубических поверхностях изучались соответственно вешественные прямые и сечения вещественными квадриками.

\section{Приложение А. Топология инволюций}

A.1. Теория Смита. Большинство результатов, приведенных в этом разделе, принадлежит П. А. Смиту; доказательства можно найти, например, в [13; гл. 3]. Всюду в этом разделе мы рассматриваем топологическое пространство $X$ с инволюцией $c: X \rightarrow X$ и полагаем $F=\operatorname{Fix} c$ и $X^{\prime}=X / c$.

A.1.1. Теорема. Следующие две последовательности, называемые (гомологической и когомологической) последовательностями Смита пространства $(X, c)$, 
естественны относительно әквивариантных отображений и точны:

$$
\begin{aligned}
& \longrightarrow H_{p+1}\left(X^{\prime}, F\right) \stackrel{\Delta}{\longrightarrow} H_{p}\left(X^{\prime}, F\right) \oplus H_{p}(F) \stackrel{\operatorname{tr}^{*}+\mathrm{in}_{*}}{\longrightarrow} H_{p}(X) \stackrel{\mathrm{pr}_{*}}{\longrightarrow} H_{p}\left(X^{\prime}, F\right) \longrightarrow \\
& \longrightarrow H^{p}\left(X^{\prime}, F\right) \stackrel{\mathrm{pr}^{*}}{\longrightarrow} H^{p}(X) \stackrel{\operatorname{tr}_{*} \oplus \mathrm{in}^{*}}{\longrightarrow} H^{p}\left(X^{\prime}, F\right) \oplus H^{p}(F) \stackrel{\Delta}{\longrightarrow} H^{p+1}\left(X^{\prime}, F\right) \longrightarrow
\end{aligned}
$$

Гомологический и когомологический связывающие гомоморфизмы $\Delta$ имеют соответственно вид

$$
x \mapsto x \cap \omega \oplus \partial x \quad u \quad x \oplus f \mapsto x \cup \omega+\delta f,
$$

где $\omega \in H^{1}\left(X^{\prime} \backslash F\right)$ - характеристический класс двулистного накрытия $X \backslash F \rightarrow$ $X^{\prime} \backslash F$. Образы гомоморфизмов $\mathrm{tr}^{*}+\mathrm{in}_{*}$ и $\mathrm{pr}^{*}$ состоят из инвариантных классов: $\operatorname{Im}_{\operatorname{tr}^{*}} \subset \operatorname{Ker}\left(1+c_{*}\right) u \operatorname{Im} \operatorname{pr}^{*} \subset \operatorname{Ker}\left(1+c^{*}\right)$.

A.1.2. Трансферь $\operatorname{tr}^{*}$ и $\operatorname{tr}_{*}$ в A.1.1 можно описать следующим образом. Представим пространство $X$ в виде $S^{0}$-расслоения, ассоциированного с относительным $D^{1}$-расслоением $T \rightarrow X^{\prime}$, характеристический класс которого равен $\omega$. А именно, возьмем цилиндр проекции $X \rightarrow X^{\prime}$ и для каждого $f \in F$ стянем слой над $f$. Тогда $\operatorname{tr}^{*}$ и $\operatorname{tr}_{*}$ являются композициями

$$
\begin{aligned}
& \operatorname{tr}^{*}: H_{p}\left(X^{\prime}, F\right) \stackrel{\mathrm{th}^{-1}}{\longrightarrow} H_{p+1}(T, X) \stackrel{\partial}{\longrightarrow} H_{p}(X), \\
& \operatorname{tr}_{*}: H^{p}(X) \stackrel{\delta}{\longrightarrow} H^{p+1}(T, X) \stackrel{\mathrm{th}^{-1}}{\longrightarrow} H^{p}\left(X^{\prime}, F\right),
\end{aligned}
$$

где th - изоморфизм Тома.

Следуюшее утверждение сразу получается из А.1.1. Мы формулируем его для гомологий, для когомологий формулировка аналогична.

А.1.3. СлЕДСТвИЕ (из А.1.1).

(1) $\operatorname{dim} H_{*}(F)+2 \sum_{p} \operatorname{dim} \operatorname{Coker}\left(\operatorname{tr}^{p}+\operatorname{in}_{p}\right)=\operatorname{dim} H_{*}(X)$ (формула Смита);

(2) $\operatorname{dim} H_{*}(F) \leqslant \operatorname{dim} H_{*}(X)$ (неравенство Смита);

(3) $\operatorname{dim} H_{*}(F) \leqslant \operatorname{dim} H^{1}\left(\mathbb{Z}_{2} ; H_{*}(X)\right)$ (неравенство Бореля-Свона);

(4) $\operatorname{dim} H_{*}(F)=\operatorname{dim} H_{*}(X) \bmod 2$;

(5) $\chi(X)=\chi(F)+2 \chi\left(X^{\prime}, F\right)$;

(6) $\chi(X)=2 \chi\left(X^{\prime}\right)-\chi(F)$.

(Напомним, что $H^{1}\left(\mathbb{Z}_{2} ; H_{*}(X)\right)=\operatorname{Ker}\left(1+c_{*}\right) / \operatorname{Im}\left(1+c_{*}\right)$.)

A.1.4. Если в A.1.3(2) достигается равенство: $\operatorname{dim} H_{*}(F)=\operatorname{dim} H_{*}(X)$, то говорят, что $c$ есть $M$-инволющия. Если в А.1.3(3) достигается равенство (что эквивалентно включению $\left.\operatorname{Im}\left(\operatorname{tr}^{*}+\operatorname{in}_{*}\right) \supset \operatorname{Ker}\left(1+c_{*}\right)\right)$, то $c$ называется $\left(\mathbb{Z}_{2^{-}}\right)$Галуа максимальной. (Эта терминология введена В. А. Красновым [70]. Р. Том [111] называет размерность $p \in \mathbb{N}$ регулярной для $(X, c)$, если $\operatorname{Im}\left(\operatorname{tr}^{p}+\operatorname{in}_{p}\right) \supset \operatorname{Ker}\left(1+c_{p}\right)$.)

В обшем случае из А.1.3(2) и (4) следует, что $\operatorname{dim} H_{*}(F)=\operatorname{dim} H_{*}(X)-2 d$ для некоторого целого $d \geqslant 0$; в этом случае $c$ назьвается $(M-d)$-инволюиией.

A.1.5. Геометрическая конструкция последовательности Смита. Введем цепные комплексы Смита

$$
\begin{aligned}
\operatorname{Sm}_{*}(X) & =\operatorname{Ker}\left[\left(1+c_{*}\right): S_{*}(X) \rightarrow S_{*}(X)\right] \\
\operatorname{Sm}_{*}(X, F) & =\operatorname{Ker}\left[\left(1+c_{*}\right): S_{*}(X, F) \rightarrow S_{*}(X, F)\right]
\end{aligned}
$$


и гомологии Смита $H_{r}\left(\mathrm{Sm}_{*}(X)\right), H_{r}\left(\mathrm{Sm}_{*}(X, F)\right)$. Имеется канонический изоморфизм $\operatorname{Sm}_{*}(X, F)=\operatorname{Im}\left[\left(1+c_{*}\right): S_{*}(X) \rightarrow S_{*}(X)\right]$ и каноническое расшепление $\operatorname{Sm}_{*}(X)=S_{*}(F) \oplus \operatorname{Im}\left(1+c_{*}\right)$. Более того, $\operatorname{tr}^{*}: S_{*}\left(X^{\prime}, F\right) \rightarrow \operatorname{Sm}_{*}(X, F)$ есть изоморфизм, и благодаря отождествлениям, которые задаются всеми этими изоморфизмами, последовательности Смита являются длинными точными гомологической и когомологической последовательностями, ассоциированными с короткой точной последовательностью комплексов

$$
0 \longrightarrow \operatorname{Sm}_{*}(X) \stackrel{\text { inclusion }}{\longrightarrow} S_{*}(X) \stackrel{1+c_{*}}{\longrightarrow} \operatorname{Sm}_{*}(X, F) \longrightarrow 0 .
$$

A.1.6. КВАДРАТЫ СТИНРОДА В ПОСЛЕДОВАТЕЛЬНОСТЯХ СМИТА. Д $x \in H_{p}\left(X^{\prime}, F\right)$ и $y \in H^{p}(X)$ справедливы равенства $\operatorname{Sq}^{*} x=\operatorname{tr}^{*} \operatorname{Sq}(x \cap(1+\omega))$ $u \operatorname{tr}_{*} \operatorname{Sq} y=\left(\operatorname{Sqtr}_{*} y\right) \cup(1+\omega)$.

ДокАЗАТЕльСтво. Это утверждение следует из А.1.2 и формулы Тома-Картана для сферических расслоений (см. [112]): поскольку тотальньй характеристический класс относительного $D^{1}$-расслоения $T \rightarrow X^{\prime}$ равен $1+\omega$, получаем, что $\operatorname{th}^{-1} \mathrm{Sq}(x \cap(1+\omega))=\mathrm{Sq}^{-1} x$ и $\mathrm{th}^{-1} \mathrm{Sq} y^{\prime}=\left(\mathrm{Sq} \mathrm{th}^{-1} y^{\prime}\right) \cup(1+\omega)$, где $y^{\prime}=\delta y$.

A.1.7. СлЕДСТВИЕ. ДЛя любих $x \in H_{p}\left(X^{\prime}, F\right)$ u $y \in H^{p}(X)$ справедливы равенства $\mathrm{Sq}_{1} \operatorname{tr}^{*} x=\operatorname{tr}^{*} \mathrm{Sq}_{1} x+\operatorname{in}_{*} \partial x u \operatorname{tr}_{*} \mathrm{Sq}^{1} y=\mathrm{Sq}_{1} \operatorname{tr}_{*} y+\delta$ in* $y$.

ДоКАЗАТЕЛЬСТво. В силу точности последовательностей Смита

$$
\operatorname{tr}^{*}(x \cap \omega)=\operatorname{in}_{*} \partial x \quad \text { и }\left(\operatorname{tr}_{*} y\right) \cup \omega=\delta \operatorname{in}^{*} y .
$$

Таким образом, доказываемые равенства следуют из А.1.6.

A.1.8. Относительный вариант последовательностей Смита. Если $A \subset X$ есть $G$-инвариантное подпространство, имеется естественная точная последовательность

$$
\begin{aligned}
& \longrightarrow H_{p+1}\left(X^{\prime}, F \cup A^{\prime}\right) \stackrel{\Delta}{\longrightarrow} H_{p}\left(X^{\prime}, F \cup A^{\prime}\right) \oplus H_{p}\left(F, F \cap A^{\prime}\right) \stackrel{\operatorname{tr}^{*}+\mathrm{in}_{*}}{\longrightarrow} \\
& \stackrel{\operatorname{tr}^{*}+\mathrm{in}_{*}}{\longrightarrow} H_{p}(X, A) \stackrel{\mathrm{pr}_{*}}{\longrightarrow} H_{p}\left(X^{\prime}, F \cup A^{\prime}\right) \longrightarrow
\end{aligned}
$$

(и такая же последовательность в когомологиях), где $A^{\prime}=A / c$. Ее свойства и их следствия аналогичны свойствам и следствиям абсолютной последовательности Смита. В частности, неравенство Смита А.1.3(2) преврашается в

$$
\operatorname{dim} H_{*}(F, F \cap A) \leqslant \operatorname{dim} H_{*}(X, A) .
$$

А.2. Спектральная последовательность Калинина. В этом разделе мы описываем относительно новое мошное средство - спектральную последовательность Калинина. Первоначально она была введена И. О. Калининым [54] как стабильный вариант спектральной последовательности Бореля-Серра расслоения $X \times_{\mathbb{Z}_{2}} S^{\infty} \rightarrow$ $\mathbb{R} \mathbb{P}^{\infty}$, где $X$ является топологическим пространством с инволюцией $c$, а $S^{\infty}$ рассматривается со стандартной антиподальной инволюцией. Эта последовательность начинается с гомологий $H_{*}(X)$ и сходится к тотальной группе гомологий $H_{*}($ Fix $c)$. Получающаяся фильтрация $\mathscr{F}^{*}$ на $H_{*}($ Fix $c)$ и изоморфизмы bv $p$ между предельным 
членом спектральной последовательности и $\mathrm{Gr}_{\mathscr{F}} H_{*}(\mathrm{Fix} c)$ были открыты O. Я. Виро геометрически до работы Калинина и первоначально были связаны с точной последовательностью Смита. Связь меж ду двумя этими конструкциями была установлена в [23]. Как показано в [26] (в предварительном варианте), а также в [29], спектральную последовательность Калинина также можно вывести из точной последовательности Смита (см. А.2.4).

Конструкция, подобная спектральной последовательности Калинина, изучалась В. А. Красновым в серии работ; см., например, [71].

Ниже мы даем другое, геометрическое, описание гомологического варианта спектральной последовательности Калинина и гомоморфизмов Виро bv * и формулируем их основные свойства. (Как обычно, когомологический вариант получается поднятием всех индексов и обращением всех стрелок; мы имеем в виду этот вариант, говоря об умножениях и двойственности Пуанкаре.) Доказательства этих результатов и/или дальнейшие подробности можно найти в [26] и [29]. Дифференциалы ${ }^{r} d_{p}$ этой спектральной последовательности и гомоморфизмы Виро bv $p$ часто рассматриваются как аддитивные отношения (т.е. как частичные многозначные гомоморфизмы) $H_{p}(X) \rightarrow H_{p+r-1}(X)$ и соответственно bv $p: H_{*}(\operatorname{Fix} c) \rightarrow H_{p}(X)$. Поскольку наш подход геометрический, нам приходится работать с цепями; в зависимости от природы пространства $X$ можно считать их сингулярными, симплициальными, гладкими и т. д. Для сходимости спектральной последовательности пространство $X$ должно удовлетворять определенным условиям, которые зависят от выбранной гомологической теории (например, пучковые (ко)гомологии и локально компактные конечномерные пространства). Конечно, эти условия всегда вьполнены для компактных гладких многообразий и конечных клеточных пространств.

Итак, зафиксируем хорошее (см. вьше) топологическое пространство $X$ с инволюцией $c$. Рассмотрим частичные гомоморфизмы bv $p: H_{*}(\operatorname{Fix} c) \rightarrow H_{p}(X)$ и $\mathbb{Z}$-градуированную спектральную последовательность $\left({ }^{r} E_{*}(X),{ }^{r} d_{*}\right)$, определенную следуюшим образом:

(1) $\mathrm{bv}_{0}$ равен нулю на $H_{\geqslant 1}(\mathrm{Fix} c)$, а его сужение на $H_{0}$ (Fix $\left.c\right)$ совпадает с гомоморфизмом включения;

(2) $\mathrm{bv}_{p}$ определен на (неоднородном) элементе $x \in H_{*}(\mathrm{Fix} c)$, представленном циклом $\sum x_{i}$ (где $x_{i}-i$-мерная компонента элемента $x$ ), тогда и только тогда, когда существуют такие цепи $y_{i}$ в $X, 1 \leqslant i \leqslant p$, что $\partial y_{1}=x_{0}$ и $\partial y_{i+1}=x_{i}+\left(1+c_{*}\right) y_{i}$ для $i \geqslant 1$; в этом случае bv $p$ классом цикла $x_{p}+\left(1+c_{*}\right) y_{p}$;

(3) ${ }^{1} E_{*}=H_{*}(X)$ и ${ }^{1} d_{*}=1+c_{*}$;

(4) ${ }^{r} d_{p}$ определен на цикле $x_{p}$ в $X$ тогда и только тогда, когда существуют такие цепи $y_{p}=x_{p}, y_{p+1}, \ldots, y_{p+r-1}$, что $\partial y_{i+1}=\left(1+c_{*}\right) y_{i}$; в этом случае ${ }^{r} d_{p} x_{p}=$ $\left(1+c_{*}\right) y_{p+r-1}$.

A.2.1. СПЕКТРАЛЬНАЯ ПОСЛЕДОВАТЕЛЬНОСТЬ ИНВОЛЮЦИИ. Гомоморфизмы $\mathrm{bv}_{*}$ и спектральная последовательность $\left({ }^{r} E_{*},{ }^{r} d_{*}\right)$ естественны относительно эквивариантных отображсений. Более того, ${ }^{r} E_{*} u^{r} d_{*}$ действительно образуют спектральную последовательность (m.е. ${ }^{r} d_{p}$ - корректно определенние гомоморфизмы $\left.{ }^{r} E_{p} \rightarrow{ }^{r} E_{p+r-1} u^{r+1} E_{p}=\operatorname{Ker}^{r} d_{p} / \operatorname{Im}^{r} d_{p-r+1}\right)$, причем эта последовательность сходится $\kappa H_{*}(\mathrm{Fix})$ посредством гомоморфизмов $\mathrm{bv}_{*}$, т.е. $\mathrm{bv}_{p}$ 
индуиирует (настоящий) изоморфизм $\mathscr{F}^{p} / \mathscr{F}^{p+1} \rightarrow{ }^{\infty} E_{p}$, где $\mathscr{F}^{p}=$ Domain $^{b v} p=$ $\operatorname{Ker}_{\mathrm{bv}} \mathrm{v}_{p-1}$.

ЗАмЕчАниЕ. Детальный анализ приведенной вьше конструкции показывает, что $\mathrm{bv}_{p}$ можно рассматривать как настояший гомоморфизм

$$
\mathrm{bv}_{p}: \operatorname{Ker}\left[\mathrm{bv}_{p-1}: H_{*}(\mathrm{Fix} c)-\rightarrow{ }^{r-1} E_{p-1}\right] \rightarrow{ }^{r} E_{p}, \quad r>1
$$

(где $\mathrm{bv}_{p}: H_{*}(\mathrm{Fix} c) \rightarrow{ }^{1} E_{p}=H_{p}(X)$ определяется на $H_{p}(\mathrm{Fix} c)$ как гомоморфизм включения и продолжается нулем на $\left.H_{>p}(\mathrm{Fix} c)\right)$. Эти гомоморфизмы, фактически, связаны с первоначальной, нестабилизированной, спектральной последовательностью Бореля-Серра (подробности см. в [23]).

Когомологический вариант ${ }^{r} E^{*} \Rightarrow H^{*}(\mathrm{Fix} c)$ строится аналогично и является двойственным гомологическому. Произведения $\cup$ и $\cap$ в $X$ после факторизации естественно переходят в структуру $\mathbb{Z}_{2}$-алгебры в ${ }^{r} E^{*}$ и соответственно в структуру ${ }^{r} E^{*}$-модуля ${ }^{r}{ }^{r} E_{*}$. Если $X-$ связное замкнутое $N$-мерное многообразие и Fix $c \neq \varnothing$, фундаментальньй класс многообразия $[X]$ доживает до ${ }^{\infty} E_{N}$ и умножение $\cap[X]:{ }^{r} E^{p} \rightarrow{ }^{r} E_{N-p}$ является изоморфизмом (двойственностью Пуанкаре). Поэтому, как обычно, определяется спаривание о: ${ }^{r} E_{p} \otimes{ }^{r} E_{q} \rightarrow{ }^{r} E_{p+q-N}$, называемое гомологическим спариванием Калинина.

A.2.2. ГОМОЛОГИЧЕСКОЕ СПАРИВАНИЕ КАЛИНИНА. Пусть $X-$ гладкое замкнутое связное $N$-мерное многообразие с гладкой инволючией $c: X \rightarrow X$. Обозначим через $w(\nu)$ тотальный класс Штифеля-Уитни нормального расслоения $\nu$ на Fix с в $X$. Тогда $w(\nu) \cap(a \circ b) \in \mathscr{F} p+q-N u$

$$
\operatorname{bv}_{p} a \circ \mathrm{bv}_{q} b=\operatorname{bv}_{p+q-N}[w(\nu) \cap(a \circ b)]
$$

$\partial \Omega_{я} a \in \mathscr{F}^{p} u b \in \mathscr{F}^{q}$.

ЗАмЕчАниЕ. Предыдущее равенство содержит в качестве специального случая вариант Бореля-Хефлигера [12] теоремы Безу над полем вешественных чисел (с $\mathbb{Z}_{2^{-}}$ значным пересечением): индекс пересечения двух вешественных алгебраических циклов $A, B$ дополнительных размерностей в вещественном алгебраическом многообразии $X$ сравним по модулю 2 с индексом пересечения циклов $\mathbb{R} A$ и $\mathbb{R} B$ в $\mathbb{R} X$. Это следует из того, что $[\mathbb{R} A]$ есть член наибольшей степени в $\mathrm{bv}^{-1}[A]$.

Гомологические операции Стинрода $\mathrm{Sq}_{t}: H_{*}(X) \rightarrow H_{*-t}(X)$ также спускаются в ${ }^{r} E_{*}$. Чтобы описать их связь с обычньми операциями Стинрода в $H_{*}(\mathrm{Fix} c)$, введем взвешенные операчии Стинрода

$$
\widehat{\mathrm{Sq}}_{t}: H_{p}(X) \rightarrow H_{\leqslant p}(X), \quad x \mapsto \sum_{0 \leqslant j \leqslant t}\left(\begin{array}{c}
P-p \\
t-j
\end{array}\right) \mathrm{Sq}_{j} x
$$

где $P>p+t$ есть степень числа 2. (Участвуюшие в определении биномиальные коэффициенты не зависят от $P$, см., например, [109; лемма I.2.6].) В результате получим: 
A.2.3. КВАДРАТЫ СТИНРОДА В СПЕКТРАЛЬНОЙ ПОСЛЕДОВАТЕЛЬНОСТИ КАлининА. Если $x \in \mathscr{F}^{p} u t \geqslant 0, m o \widehat{\mathrm{Sq}}_{t} x \in \mathscr{F} p-t u$

$$
\mathrm{Sq}_{t} \mathrm{bv}_{p} x=\mathrm{bv}_{p-t} \widehat{\mathrm{Sq}}_{t} x \text {. }
$$

Спектральную последовательность Калинина можно, в свою очередь, вывести из точной последовательности Смита А.1.1. Ниже мы даем соответствующее описание дифференциалов ${ }^{r} d_{*}$ и гомоморфизмов Виро $\mathrm{bv}_{*}$; построение спектральной последовательности и фильтрации $\mathscr{F}^{*}$ можно найти в [29].

A.2.4. СПЕКТРАЛЬНАЯ ПОСЛЕДОВАТЕЛЬНОСТЬ КАЛИНИНА И ТОЧНАЯ ПОСЛЕДОВАТЕЛЬНОСТЬ СмИТА. Дифференииаль ${ }^{r} d_{p}$ и гомоморфизмы Виро bv $р$ задаются равенствами

$$
\begin{gathered}
{ }^{r} d_{p}=\operatorname{tr}^{p+r-1} \circ \Delta_{p+r-1}^{-1} \circ \cdots \circ \Delta_{p+1}^{-1} \circ \operatorname{pr}_{p}: H_{p}(X) \rightarrow H_{p+r-1}(X), \\
\operatorname{bv}_{p}\left(\sum_{r \leqslant p} x_{r}\right)=\operatorname{in}_{p} x_{p}+\operatorname{tr}^{p} y_{p}^{\prime}: H_{*}(\operatorname{Fix} c) \rightarrow H_{p}(X),
\end{gathered}
$$

где $\operatorname{in}_{*}: H_{*}(\operatorname{Fix} c) \rightarrow H_{*}(X)-$ гомоморфизм включения, $\operatorname{tr}^{*}, \operatorname{pr}_{*} u \Delta_{*}-$ такие же, как в точной последовательности Смита А.1.1, а в последнем равенстве $x_{r} \in H_{r}(\operatorname{Fix} c)$ и $y_{p}^{\prime} \in H_{p}\left(X^{\prime}, \operatorname{Fix} c\right)$ определяются индуктивно: $y_{0}^{\prime}=0, y_{r+1}^{\prime}=$ $\Delta_{r+1}^{-1}\left(x_{r} \oplus y_{r}^{\prime}\right) \in H_{r+1}\left(X^{\prime}, \operatorname{Fix} c\right)$.

A.2.5. Поднятие инволюций. Пусть $(X, c)$ - топологическое пространство с инволюцией, $Y \rightarrow X-$ двулистное накрытие и $\omega \in H^{1}(X)$ - его характеристический класс. Тогда $c$ поднимается до инволюции на $Y$ тогда и только тогда, когда $\omega$ доживает до ${ }^{\infty} E^{1}(X)$. Если это условие выполнено, существуют, очевидно, две инволюции $c_{1}, c_{2}: Y \rightarrow Y$, накрывающие $c$, которые коммутируют друг с другом и с автоморфизмом накрытия $\tau$ и удовлетворяют равенству $c_{1} \circ c_{2}=\tau$.

Очевидное необходимое условие доживания класса $\omega$ до ${ }^{\infty} E^{1}$ состоит в том, что он должен быть $c^{*}$-инвариантным (т.е. доживать до ${ }^{2} E^{1}$ ). Заметим, что если $X$ связно и Fix $c \neq \varnothing$, это условие также и достаточно, поскольку все дифференциалы, определенные на ${ }^{r} E^{0}$, тривиальны.

Все сказанное выше применимо и к разветвленным накрытиям с заменой пространства $X$ на $X \backslash A$, где $A$ - множество ветвления.

А.2.6. Комплексные поверхности. Спектральная последовательность Калинина, а также связанные с ней гомологическое спаривание и операции Стинрода имеют ясное геометрическое истолкование в случае, когда $X$ есть комплексная поверхность с вещественной структурой $c=$ conj. Пусть $C_{i}, i=1, \ldots, k,-$ компоненты множества $\mathbb{R} X,\left\langle C_{i}\right\rangle \in H_{0}(\mathbb{R} X)$ - соответствуюшие классы и $C_{I}=\sum_{i \in I}\left\langle C_{i}\right\rangle$ для

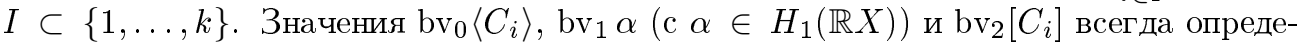
лены и совпадают с образами этих классов при гомоморфизмах включения. Значение $\mathrm{bv}_{1}\left\langle C_{I}\right\rangle$ определено, если число $|I|$ слагаемых четно; оно представляется набором $\left(1+\operatorname{conj}_{*}\right) y_{1}$ инвариантных окружностей, где $y_{1}-$ набор дуг в $X$, множество концов которых распределено между слагаемыми $C_{i}, i \in I$, по одному в каждом слагаемом. Если для такого набора $y_{1}$ кривая $\left(1+\right.$ conj $\left._{*}\right) y_{1}$ гомологична нулю: $\left(1+\operatorname{conj}_{*}\right) y_{1}=\partial y_{2}$, то $\mathrm{bv}_{1}\left\langle C_{I}\right\rangle=0$ и цикл $\left(1+\operatorname{conj}_{*}\right) y_{2}$ представляет класc $\mathrm{bv}_{2}\left\langle C_{I}\right\rangle$. Аналогично, если 
bv $_{1} \alpha=0$, т.е. цикл $\alpha$ гомологичен $\left(1+\operatorname{conj}_{*}\right) y_{1}$ для некоторой цепи $y_{1}$ в $X$, существует такая цепь $y_{2}$ в $X$, что $\partial y_{2}=\alpha+\left(1+\operatorname{conj}_{*}\right) y_{1}$, и цикл $\left(1+\right.$ conj $\left._{*}\right) y_{2}$ представляет класc $\mathrm{bv}_{2} \alpha$. Наконец, если $\mathrm{bv}_{1} \alpha=\mathrm{bv}_{1}\left\langle C_{I}\right\rangle$ для некоторого 0-класса $C_{I}$ и 1-класса $\alpha \in H_{1}(\mathbb{R} X)$, то класc $\operatorname{bv}_{2}\left(\alpha+\left\langle C_{I}\right\rangle\right)$ определен и представлен циклом $\left(1+\operatorname{conj}_{*}\right) y_{2}$, где $\partial y_{2}=\alpha+\left(1+\right.$ conj $\left._{*}\right) y_{1}$ и $y_{1}-$ набор дуг с таким же, как и выше, условием на их концы.

Элементы вида $\mathrm{bv}_{2}\left[C_{i}\right], \mathrm{bv}_{2}\left\langle C_{I}\right\rangle, \mathrm{bv}_{2} \alpha$ и $\mathrm{bv}_{2}\left(\alpha+\left\langle C_{I}\right\rangle\right)$ порождают ${ }^{\infty} E_{2}(X) ;$ злоупотребляя обозначениями, мы полагаем $\sum \mathrm{bv}_{2} x_{j}=\mathrm{bv}_{2} \sum x_{j}$ при условии, что правая часть определена, хотя каждое слагаемое слева не обязательно определено. Согласно А.2.2 гомологическая форма Калинина на ${ }^{\infty} E_{2}(X)$ задается табл. 4 , где пересечение $\alpha \circ \beta$ рассматривается как элемент групшы $H_{0}(\mathbb{R} X), \operatorname{aug}(\alpha \circ \beta)$ и $\operatorname{aug}_{i}(\alpha \circ \beta)$ являются соответственно обшим индексом пересечения и его частью, попадающей в $C_{i}$. Знаком $\delta_{i j}$ обозначается символ Кронекера: $\delta_{i i}=1$ и $\delta_{i j}=0$, если $i \neq j$. Эта форма продолжается линейно на классы вида $\mathrm{bv}_{2}\left(\alpha+\left\langle C_{I}\right\rangle\right)$, как если бы bv $2 \alpha$ и $\mathrm{bv}_{2}\left\langle C_{I}\right\rangle$ были определены.

\begin{tabular}{|c|c|c|c|}
\hline & $\mathrm{bv}_{2}\left\langle C_{I}\right\rangle$ & $\mathrm{bv}_{2} \alpha$ & $\mathrm{bv}_{2}\left[C_{i}\right]$ \\
\hline $\mathrm{bv}_{2}\left\langle C_{K}\right\rangle$ & 0 & 0 & $\sum_{k \in K} \delta_{i k}$ \\
\hline $\mathrm{bv}_{2} \beta$ & 0 & $\operatorname{aug}(\alpha \circ \beta)$ & $\operatorname{aug}_{i}(\beta \circ \beta)$ \\
\hline $\mathrm{bv}_{2}\left[C_{k}\right]$ & $\sum_{i \in I} \delta_{i k}$ & $\operatorname{aug}_{k}(\alpha \circ \alpha)$ & $\delta_{i k} \chi\left(C_{i}\right)$ \\
\hline
\end{tabular}

ТАБлицА 4. Гомологическая форма Калинина

Гомоморфизм Бокштейна $\mathrm{Sq}_{1}:{ }^{\infty} E_{2}(X) \rightarrow{ }^{\infty} E_{1}(X)$ определяется с помощью А.2.3: $\mathrm{Sq}_{1} \mathrm{bv}_{2}\left[C_{i}\right]=\mathrm{bv}_{1} w_{1}\left(C_{i}\right)$ и $\mathrm{Sq}_{1} \mathrm{bv}_{2}\left(\alpha+\left\langle C_{I}\right\rangle\right)=\mathrm{bv}_{1} \alpha$.

В заключение этого раздела мы даем несколько примеров выгисления фильтрации $\mathscr{F}^{*}$ и гомоморфизмов Виро $\mathrm{bv}_{*}$. Первый пример может служить моделью регулярных полных пересечений в $\mathbb{P}^{n}$ (см. [54]), второй - локальной моделью инволюций на гладких многообразиях.

A.2.7. Фильтрации в $\mathbb{R}^{n}$ (см. [54]). Пусть $X=\mathbb{P}^{n} u c=$ conj. Тогда фильтрации $\mathscr{F}^{*}$ на $H_{*}\left(\mathbb{R P}^{n}\right)$ и $\mathscr{F}_{*}$ на $H^{*}\left(\mathbb{R P}^{n}\right)$ имеют вид

$$
\mathscr{F}^{r}=\mathrm{Sq} H_{\geqslant r / 2}\left(\mathbb{R P}^{n}\right), \quad \mathscr{F}_{r}=\mathrm{Sq} H^{\leqslant r / 2}\left(\mathbb{R P}^{n}\right),
$$

где через Sq обозначаются как гомологический, так и когомологический тотальные квадраты Стинрода.

A.2.8. ФИЛЬТРАцИЯ В ПРОСТРАНСТВАХ ТОМА (см. [26]). Пусть $\nu$ ecmb m-мерное векторное расслоение над конечным клеточным пространством $F$, и пусть T и $Т$ - ассоциированные расслоения соответственно на шары и сферьи, наделеннье антиподальной инволючией. Тогда фильтрации $\mathscr{F}^{*}$ на $H_{*}(F)$ и $\mathscr{F}_{*}$ на $H^{*}(F)$, ассочиированные со спектральной последовательностью Калинина пары $(T, \partial T)$, задаются равенствами

$$
\mathscr{F}^{p}=w(\nu)^{-1} \cap H_{\geqslant p-m}(F), \quad \mathscr{F}^{p}=w(\nu) \cup H^{\leqslant p-m}(F),
$$


əде $w(\nu)=1+w_{1}(\nu)+w_{2}(\nu)+\cdots-$ тотальный класс Штифеля-Уитни расслоения $\nu$. Гомоморфизмами Виро являются

$$
\begin{gathered}
\mathrm{bv}_{p}: \mathscr{F}^{p} \rightarrow H_{p}(T, \partial T), \quad x \mapsto \mathrm{th}^{-1}[w(\nu) \cap x]_{p-m}, \\
\mathrm{bv}^{p}: H^{p}(T, \partial T) \rightarrow H^{*}(F) / \mathscr{F}_{p-1}, \quad x \mapsto w(\nu) \cup \mathrm{th}^{-1} x,
\end{gathered}
$$

где th - изоморфизм Тома, $a[x]_{r}-r$-мерная компонента неоднородного гомологического класса $x$.

Следующее утверждение является обобшением утверждения А.2.7; его можно вывести из А.2.7, используя принцип расщепления.

A.2.9. ФИЛЬТРАЦИИ НА МНОГООБРАЗИЯх ГРАССмана. Пусть $X=G_{k}\left(\mathbb{C}^{n}\right)-$ многообразие Грассмана k-мерных плоскостей в $\mathbb{C}^{n}$ и с $=$ conj - стандартное сопряжсение. Тогда фильтрации $\mathscr{F}^{*}$ на $H_{*}\left(G_{k}\left(\mathbb{R}^{n}\right)\right)$ и $\mathscr{F}_{*}$ на $H^{*}\left(G_{k}\left(\mathbb{R}^{n}\right)\right)$ имеют вид

$$
\mathscr{F}^{r}=\operatorname{Sq} H_{\geqslant r / 2}\left(G_{k}\left(\mathbb{R}^{n}\right)\right), \quad \mathscr{F}_{r}=\operatorname{Sq} H^{\leqslant r / 2}\left(G_{k}\left(\mathbb{R}^{n}\right)\right),
$$

где через $\mathrm{Sq}$ обозначаются как гомологический, так и когомологический тотальнье квадрать Стинрода.

Следующее следствие утверждения А.2.9, по существу, равносильно ему. Другое связанное с этим тождество имеется в [71].

A.2.10. СЛЕДСТВИЕ. Пусть $X$ - топологическое пространство с инволюиией $c: X \rightarrow X$ и $E$ - пространство вещественного векторного расслоения над $X$ в смысле Атийи [7] (т.е. E - пространство комплексного векторного расслоения, наделенное антилинейной инволючией $c_{E}$, накрывающей инволючию $\left.c\right)$. Тогда $\mathrm{bv}^{2 i} c_{i}(E)=\mathrm{Sq}^{i}(\mathbb{R} E)$ для всех $i$, где $\mathbb{R} E=\operatorname{Fix}_{E}-$ пространство вещественного векторного расслоения над Fix $c$.

A.3. Инволюции на многообразиях. Пусть $X$ - гладкое замкнутое ориентированное $2 k$-мерное многообразие и $c$ - гладкая инволюция, сохраняющая ориентацию, если $k$ четно, и обрашающая ее в противном случае. Определим скрученную форму пересечений

$$
B^{c}:\left(H_{k}(X ; \mathbb{Z}) / \text { Tors }\right) \otimes\left(H_{k}(X ; \mathbb{Z}) / \text { Tors }\right) \rightarrow \mathbb{Z}, \quad B^{c}(x, y)=x \circ c_{*} y
$$

Эта форма симметрична; ее сигнатура $\sigma(c)$ называется сигнатурой инволюиии. Если $k$ нечетно, сигнатура равна 0 , в противном случае $\sigma(c)=\sigma_{+}-\sigma_{-}$, где $\sigma_{ \pm}-$сигнатуры сужений формы пересечений на собственные подпространства $H_{k}^{ \pm 1}$ инволюции $c_{*}$.

A.3.1. ТеОрема. Если все компоненты множсества $F=$ Fix с имеют размерность $k$, то $\sigma(c)=F \circ F$, əде $F \circ F$ - нормальное число Эйлера подмногообразия $F$ в $X$.

Поскольку вещественная часть $\mathbb{R} X$ неособого комплексного многообразия $X$ с вещественной структурой вполне вешественна, все компоненты множества $\mathbb{R} X$ имеют размерность $n=\operatorname{dim}_{\mathbb{C}} X$ и потому $\mathbb{R} X \circ \mathbb{R} X=(-1)^{n / 2} \chi(\mathbb{R} X)$. Если $X$ наделено инвариантной кэлеровой метрикой, вешественная часть является лагранжевой. 
A.3.2. СлЕДСтвиЕ. Если $X-2 k$-мерное неособое компактное комплексное многообразие с вещественной структурой conj, то $\sigma(\mathrm{conj})=(-1)^{k} \chi(\mathbb{R} X)$.

Если $X$ - замкнутое компактное (не обязательно ориентируемое) многообразие, размерность которого $\operatorname{dim} X=2 k$ четна, определен $\mathbb{Z}_{2}$-значный вариант $B^{c}: H_{k}(X) \otimes$ $H_{k}(X) \rightarrow \mathbb{Z}_{2}$ скрученной формы пересечений. Эта форма также симметрична.

A.3.3. ЛЕмма АрнольДа. Пусть $X$ - замкнутое гладкое $2 k$-мерное многообразие с инволюиией $c$. Если $\operatorname{dim} \operatorname{Fix} c \leqslant k$, то фундаментальный класс $[\mathrm{Fix} c]_{k}$ объединения $k$-мерных компонент множества Fix с реализует в $H_{k}(X)$ характеристический класс форми $B^{c}: H_{k}(X) \otimes H_{k}(X) \rightarrow \mathbb{Z}_{2}$.

ЗАмЕчАниЕ. В общем случае характеристический класс формы $B^{c}$ есть $k$-мерная компонента класса in! $\left(v(\tau) v^{-1}(\nu)\right)$, где in: Fix $c \rightarrow X$ - включение, $\tau$ и $\nu$-касательное и соответственно номальное расслоения на Fix $c$, a $v$ обозначает тотальньй класс Ву. Подробности см. в [21].

\section{Приложение В. Целочисленные решетки и квадратичные формы}

В этом приложении мы даем краткое введение в арифметику целочисленных решеток и конечных квадратичных форм, которая часто используется в топологии вешественных алгебраических многообразий. Доказательства, в большинстве несложные, можно найти в любом учебнике, например, в [85] и [104].

B.1. Конечные квадратичные формы. Конечной симметричной билинейной формой называется пара $(\mathscr{L}, b)$, где $\mathscr{L}$ - конечная абелева группа и $b: \mathscr{L} \otimes \mathscr{L} \rightarrow$ $\mathbb{Q} / \mathbb{Z}$ - симметричная билинейная форма. Конечной квадратичной формой на $\mathscr{L}$ называется отображение $q: \mathscr{L} \rightarrow \mathbb{Q} / 2 \mathbb{Z}$, удовлетворяющее условиям $q(n x)=n^{2} q(x)$, $x \in \mathscr{L}, n \in \mathbb{Z}$, и $q(x+y)-q(x)-q(y)=2 b(x, y), x, y \in \mathscr{L}$, где $b$ - симметричная билинейная форма; форма $b$ определяется формой $q$ и называется билинейной формой, ассоииированной $c$, в свою очередь $q$ назьвается квадратичныц расиирением формы $b$.

В случаях, не вызывающих недоразумений, для квадратичной формы $q$ (билинейной формы $b$ ) мы часто используем сокрашенное обозначение $q(x)=x^{2}$ (соответственно $b(x, y)=x y)$.

Если $q_{1}, q_{2}$ - два квадратичных расширения одной и той же билинейной формы $b$, разность $q_{1}-q_{2}$ есть линейная форма. Более того, $q_{i}(x)=b(x, x) \bmod \mathbb{Z}, i=1,2$, для любого $x \in \mathscr{L} ;$ поэтому $q_{1}-q_{2}$ принимает значения в $\mathbb{Z}_{2}=\mathbb{Z} / 2 \mathbb{Z} \subset \mathbb{Q} / 2 \mathbb{Z}$. Следовательно, множество $\mathrm{Qu}(\mathscr{L}, b)$ квадратичных расширений данной билинейной формы $b$ на $\mathscr{L}$ является афффинным пространством над $\operatorname{Hom}\left(\mathscr{L}, \mathbb{Z}_{2}\right)$. В частности, если $\mathscr{L}$ не имеет 2 -кручения, любая билинейная форма $b$ на $\mathscr{L}$ имеет единственное квадратичное расширение.

Симметричная билинейная форма $b$ называется невырожденной, если гомоморфизм корреляции $\mathscr{L} \rightarrow \operatorname{Hom}(\mathscr{L}, \mathbb{Q} / \mathbb{Z}), x \mapsto b(x, \cdot)$, биективен. Квадратичная форма назьвается невырожденной, если невырождена ассоциированная с ней билинейная форма. В обшем случае ядро гомоморфизма корреляции обозначается через $\operatorname{Ker} \mathscr{L}$ или $\mathscr{L}^{\perp}$.

Невырожденные конечные квадратичные (билинейные) формы образуют аддитивный моноид Bil (соответственно Qu) с операцией ортогонального суммирования. Сис- 
тема образуюших моноида Bil указана, например, в [121]; для каждой образуюшей группа $\mathscr{L}$ либо циклическая, либо является (не ортогональной) суммой двух циклических групп. Образуюшие моноида $\mathrm{Qu}$ можно получить поднятием образуюших моноида Bil. В этом обзоре мы имеем дело исключительно с формами на группах периода 2 (однако доказательства результатов о вешественных поверхностях Энриквеса сушественно используют формы на группах периода 4 , теорию которых можно найти в [29]); они образуют прямые слагаемые $\mathrm{Bil}_{2}$ и $\mathrm{Qu}_{2}$ моноидов $\mathrm{Bil}$ и $\mathrm{Qu}$ соответственно. Для $\mathrm{Bil}_{2}$ образуюшими Уолла являются

$$
\mathscr{L}=\mathbb{Z}_{2}: \quad a_{2}=\left[\frac{1}{2}\right] ; \quad \mathscr{L}=\mathbb{Z}_{2} \times \mathbb{Z}_{2}: \quad u_{2}=\left[\begin{array}{cc}
0 & \frac{1}{2} \\
\frac{1}{2} & 0
\end{array}\right] .
$$

Каждая из форм $a_{2}$ и $u_{2}$ имеет с точностью до изоморфизма два квадратичных расширения:

$$
\text { для } a_{2}:\left[ \pm \frac{1}{2}\right], \quad \text { для } u_{2}: \mathscr{U}_{2}=\left[\begin{array}{cc}
0 & \frac{1}{2} \\
\frac{1}{2} & 0
\end{array}\right] \text { и } \mathscr{V}_{2}=\left[\begin{array}{cc}
1 & \frac{1}{2} \\
\frac{1}{2} & 1
\end{array}\right] .
$$

Инвариантом Брауна $\operatorname{Br} q$ невырожденной конечной квадратичной формы $q$ на $\mathscr{L}$ назьвается класс вычетов по модулю 8 , определяемый равенством

$$
\exp \left(\frac{1}{4} i \pi \operatorname{Br} q\right)=|\mathscr{L}|^{-\frac{1}{2}} \sum_{x \in \mathscr{L}} \exp (i \pi q(x)) .
$$

В.1.1. Справедливы равенства $\operatorname{Br}\left[ \pm \frac{1}{2}\right]= \pm 1, \operatorname{Br} \mathscr{U}_{2}=0$ и $\mathrm{Br}^{\mathscr{V}} / 2=4$.

Конечная группа $\mathscr{L}$ периода 2 является $\mathbb{Z}_{2}$-векторным пространством. Пространство $\mathscr{L}$ с конечной квадратичной формой на нем называется квадратичным $\mathrm{npocm-}$ ранством. Квадратичная (билинейная) форма на $\mathbb{Z}_{2}$-пространстве принимает значения в $\left(\frac{1}{2} \mathbb{Z}\right) / 2 \mathbb{Z} \subset \mathbb{Q} / 2 \mathbb{Z}$ (соответственно в $\left(\frac{1}{2} \mathbb{Z}\right) / \mathbb{Z} \subset \mathbb{Q} / \mathbb{Z}$ ). Используя канонические отождествления $\left(\frac{1}{2} \mathbb{Z}\right) / 2 \mathbb{Z}=\mathbb{Z}_{4}$ и $\left(\frac{1}{2} \mathbb{Z}\right) / \mathbb{Z}=\mathbb{Z}_{2}, \frac{1}{2} \mapsto 1$, можно рассматривать квадратичные (билинейные) формы на $\mathbb{Z}_{2}$-пространствах как отображения $\mathscr{L} \rightarrow \mathbb{Z}_{4}$ (соответственно $\mathscr{L} \otimes \mathscr{L} \rightarrow \mathbb{Z}_{2}$ ).

Вектор $w \in \mathscr{L}$ назьвается характеристическим әлементом пространства $\mathscr{L}$, если $x w=x^{2} \bmod 2$ для любого $x \in \mathscr{L}$. Поскольку на группах периода 2 отображение $x \mapsto x^{2} \bmod 2$ линейно, характеристические элементы сушествуют и образуют класс смежности $\bmod (\operatorname{Ker} \mathscr{L})$; он называется характеристическим классом смежности пространства $\mathscr{L}$. Если $\mathscr{L}$ невырождено, характеристический элемент единственен. Подгруппа в $\mathscr{L}$, натянутая на объединение ядра $\operatorname{Ker} \mathscr{L}$ и характеристического элемента, будет обозначаться через $\Omega=\Omega(\mathscr{L})$.

Квадратичное пространство называется четным, если 0 является его характеристическим элементом, т.е. если $x^{2}=0 \bmod 2$ для любого $x \in \mathscr{L}$. В противном случае оно называется нечетныц.м.

B.1.2. Моноид невырожденных конечных квадратичных форм на группах периода 2 порожден формами $\mathscr{A}^{ \pm}=\left[ \pm \frac{1}{2}\right], \mathscr{U}_{2}$ и $\mathscr{V}_{2}$, которье подчинены соотношениям $2 \mathscr{U}_{2}=2 \mathscr{V}_{2}, 3 \mathscr{A}^{ \pm}=\mathscr{A}^{\mp} \oplus \mathscr{V}_{2}$ и $\mathscr{A}^{ \pm} \oplus \mathscr{A}^{+} \oplus \mathscr{A}^{-}=\mathscr{A}^{ \pm} \oplus \mathscr{U}_{2}$. 
Два невырожсденных квадратичных пространства изоморфны тогда и только тогда, когда они имеют одинаковые ранг, инвариант Брауна и оба либо четнъ, либо нечетны.

Квадратичная форма $q$ на $\mathscr{L}$ назьвается информативной, если $\left.q\right|_{\mathscr{L} \perp}=0$. Понятие инварианта Брауна распространяется на информативные формы: поскольку $q$ равна нулю на $\mathscr{L}^{\perp}$, она факторизуется до квадратичной формы $q^{\prime}: \mathscr{L} / \mathscr{L}^{\perp} \rightarrow \mathbb{Q} / 2 \mathbb{Z}$, и можно положить $\operatorname{Br} q=\operatorname{Br} q^{\prime}$. Инвариант Брауна аддитивен: для любой пары $\left(\mathscr{L}_{i}, q_{i}\right)$, $i=1,2$, информативных квадратичных форм $\operatorname{Br}\left(\mathscr{L}_{1} \oplus \mathscr{L}_{2}, q_{1} \oplus q_{2}\right)=\operatorname{Br}\left(\mathscr{L}_{1}, q_{1}\right)+$ $\operatorname{Br}\left(\mathscr{L}_{2}, q_{2}\right)$.

Подформа $\mathscr{N}$ информативной квадратичной формы $(\mathscr{L}, q)$ называется информативной, если $\left.\mathscr{N}^{\perp} \subset \mathscr{N}_{\text {и }} q\right|_{\mathscr{N} \perp}=0$. (Информативная подформа является, очевидно, информативной формой; поэтому для нее определен инвариант Брауна.) Подформа $\mathscr{S} \subset \mathscr{L}$ называется изотропной (точнее, $q$-изотропной), если $\left.q\right|_{\mathscr{S}}=0$. Ясно, что подформа $\mathscr{N}$ невырожденной формы $(\mathscr{L}, q)$ информативна тогда и только тогда, когда $\mathscr{N}^{\perp}$ изотропна.

Следуюшее утверждение сразу получается из свойств суммы Гаусса.

B.1.3. Если $\mathscr{S}$ - q-изотропная подформа невырожденной квадратичной формы $(\mathscr{L}, q)$, то q определяет невырожсденную квадратичную форму на $\mathscr{S}^{\perp} / \mathscr{S}$, причем $\operatorname{Br}\left(S^{\perp} / S\right)=\operatorname{Br} \mathscr{L}$.

B.1.4. СлЕДСТВИЕ. Если $\mathscr{N}$ - информативная подформа информативной квадратичной формы $(\mathscr{L}, q)$, то $\operatorname{Br}\left(\mathscr{N},\left.q\right|_{\mathcal{N}}\right)=\operatorname{Br}(\mathscr{L}, q)$.

ЗАмЕЧАНИЕ. Понятие информативного подпространства имеет смысл и в случае, когда квадратичная форма $q$ определена лишш на $\mathscr{N}$ (но при этом билинейная форма определена на всем пространстве $\mathscr{L}$ ). В этом случае следствие В.1.4 утверждает, что $\operatorname{Br} q^{\prime}=\operatorname{Br} q$ для любого расшшрения $q^{\prime}$ формы $q$ на $L$.

B.1.5. Для любого информативного квадратичного пространства $(\mathscr{L}, q)$ :

(1) $\operatorname{Br} q=\operatorname{dim}\left(\mathscr{L} / \mathscr{L}^{\perp}\right) \bmod 2$;

(2) $\operatorname{Br} q=q(w) \bmod 4$ для любого характеристического әлемента $w \in \mathscr{L}$;

(3) $\operatorname{Br}(q+v)=\operatorname{Br} q-2 q(v)$ для любого $v \in \mathscr{L}$ (где $q+v$ обозначает квадратичную форму $x \mapsto q(x)+2(v x))$;

(4) $\operatorname{Br} q=0$ тогда и только тогда, когда форма $(\mathscr{L}, q)$ кобордантнанулю, т.е. существует такое подпространство $\mathscr{H} \subset \mathscr{L}$, что $\mathscr{H}^{\perp}=\mathscr{H}$ u $\left.q\right|_{\mathscr{H}}=0$.

В.2. Целочисленные решетки. (Целочисленной) решеткой называется свободная абелева группа $L$ конечного ранга вместе с симметричной билинейной формой $b: L \otimes L \rightarrow \mathbb{Z}$. Как и в случае конечных форм, мы часто используем сокрашенные обозначения $b(x, y)=x y$ и $b(x, x)=x^{2}$. Решетка $L$ назьвается четной, если $x^{2}=0 \bmod 2$ для любого $x \in L$; в противном случае $L$ называется нечетной. Пусть $L^{\vee}=\operatorname{Hom}(L, \mathbb{Z})$ - двойственная абелева группа. Решетка $L$ назьвается невырожденной (унимодулярной), если гомоморфизм корреляиии $L \rightarrow L^{\vee}, x \mapsto b(x, \cdot)$, инъективен (соответственно биективен). Коядро гомоморфизма коррелящии назьвается дискриминантной группой решетки $L$ и обозначается через $\operatorname{discr} L$ или $\mathscr{L}$.

Билинейная форма на $L$ продолжается до $\mathbb{Q}$-значной билинейной формы на $L \otimes \mathbb{Q}$. Если $L$ невырождена, имеется естественное отождествление $L^{\vee}=\{x \in L \otimes \mathbb{Q} \mid x y \in \mathbb{Z}$ 
для $y \in L\}$ и гомоморфизм коррелящии является вложением $L \hookrightarrow L^{\vee}$. Поэтому група $\mathscr{L}$ конечна, а форма на $L$ продолжается до $\mathbb{Q}$-значной формы на $L^{\vee}$, которая, в свою очередь, индуцирует конечную симметричную билинейную форму $b: \mathscr{L} \otimes \mathscr{L} \rightarrow \mathbb{Q} / \mathbb{Z}$. Порядок групшы $\mathscr{L}$ равен модулю определителя $\operatorname{det} L$ матрицы Грама решетки $L$. Если к тому же $L$ четна, то форма $b$ является ассоциированной с конечной квадратичной формой $q: \mathscr{L} \rightarrow \mathbb{Q} / 2 \mathbb{Z}$, определенной равенством $q(x+L)=x^{2} \bmod 2 \mathbb{Z}, x \in L^{\vee}$.

B.2.1. ФОРМУЛА ВАН ДЕР БЛИЯ. Если $L$ - невырожденная четная целочисленная решетка, то $\operatorname{Br} \mathscr{L}=\sigma(L) \bmod 8$.

Следующее предложение сразу получается из В.2.1.

B.2.2. СлЕДСТВИЕ. Пусть $L$ - четная невырожсденая целочисленная решетка, дискриминантная группа которой $\mathscr{L}=\operatorname{discr} L$ имеет период 2. Тогда:

(1) если L унимодулярна (m.е. $\mathscr{L}=0), \operatorname{mo} \sigma(L)=0 \bmod 8$;

(2) ecлu det $L= \pm 2$ (m.e. $\mathscr{L}=\mathbb{Z}_{2}$ ), mo $\sigma(L)= \pm 2 \bmod 8$;

(3) если $\operatorname{det} L= \pm 4$ (т.е. $\mathscr{L}=\mathbb{Z}_{2} \oplus \mathbb{Z}_{2}$ ) и дискриминантная форма на $\mathscr{L}$ нечетна, то $\sigma(L)=0, \pm 2 \bmod 8$;

(4) если дискриминантная форма на $\mathscr{L}$ четна, то $\sigma(L)=0 \bmod 4$.

B.3. Инволюции на унимодулярных решетках. Пусть $L$ - унимодулярная решетка и $c: L \rightarrow L$ - инволютивная автоизометрия решетки $L$. Для подрешеток $L^{ \pm} \subset L$, инвариантных и косоинвариантных относительно $c$ элементов, положим $q^{ \pm}=\operatorname{discr} L^{ \pm}$и $J=L /\left(L^{+} \oplus L^{-}\right)$. Как $\mathbb{Z}_{2}$-векторные пространства $J$ и $q^{ \pm}$изоморфны. Подрешетки $L^{ \pm}$являются ортогональньми дополнениями друг друга, причем $\operatorname{rk} L^{+}+\operatorname{rk} L^{-}=\operatorname{rk} L$ и

$$
\log _{2}\left|\operatorname{discr} L^{ \pm}\right|=\operatorname{dim}_{\mathbb{Z}_{2}} J=\operatorname{dim}_{\mathbb{Z}_{2}} L \otimes \mathbb{Z}_{2}-\operatorname{dim}_{\mathbb{Z}_{2}}\left\{L \otimes \mathbb{Z}_{2}\right\}^{c},
$$

где $\left\{L \otimes \mathbb{Z}_{2}\right\}^{c}=\left\{x \in L \otimes \mathbb{Z}_{2} \mid c x=l\right\}$.

Легко проверить, что справедливо следуюшее утверждение.

B.3.1. Форма $q^{+}$четна тогда и только тогда, когда характеристические әлементы решетки L совпадают с характеристическими әлементами скрученной формы $b^{c}: L \otimes L \rightarrow \mathbb{Z}_{2}, x \otimes y \mapsto b(x, c y)$.

\section{Приложение С. Форма Рохлина-Гийю-Марена}

В этом приложении мы кратко напоминаем конструкцию формы Рохлина-ГийюМарена (см. [46]) характеристической поверхности в четырехмерном многообразии.

Пусть $Y$ - ориентированное замкнутое гладкое четырехмерное многообразие и $U$ характеристическая поверхность в $Y$, т.е. гладкое замкнутое двумерное подмногообразие с $[U]=\mathrm{D} u_{2}(Y)$ в $H_{2}(Y)$. Обозначим через $i: U \hookrightarrow Y$ включение и положим $K=\operatorname{Ker}\left[i_{*}: H_{1}(U) \rightarrow H_{1}(Y)\right]$. Тогда сушествует естественное квадратичное расширение $\mathfrak{g m}: K \rightarrow \mathbb{Z}_{4}$ гомологической формы пересечений, которое называется формой Рохлина-Гийю-Марена пары $(Y, U)$. Геометрически ее можно определить следующим образом. Возьмем класс $x \in K$ и реализуем его объединением l попарно не пересекаюшихся простых замкнутых гладких кривых в $U$. Оно является краем погруженной в $Y$ поверхности $\mathfrak{M}$, которую можно выбрать нормальной к $U$ вдоль $\mathfrak{l}=\partial \mathfrak{M}$ и трансверсальной к $U$ в ее внутренних точках. (Такая поверхность назьвается перепонкой.) 
Рассмотрим поле нормальных прямых $\xi$ на $\mathfrak{l}$, касательных к $U$, и определим индекс ind $\mathfrak{M} \in \mathbb{Z}$ как препятствие к продолжению поля $\xi$ до поля нормальных прямых на $\mathfrak{M}$. (Поскольку $\tau \mathfrak{M} \oplus \nu \mathfrak{M}$ - ориентированное векторное расслоение, это препятствие определено и является целым числом. Если кривая $\mathfrak{l}$ расположена двусторонне в $U$, этот индекс равен удвоенному индексу, определенному с помошью векторных полей вместо полей прямых.) Тогда $\mathfrak{g m}(x)=$ ind $\mathfrak{M}+2 \operatorname{Card}($ int $\mathfrak{M} \cap F) \bmod 4$.

C.1. ТеОРемА. Для указанных $Y$ и U пара $(K, \mathfrak{g m})$ является информативным подпространством в $H_{1}(U)$ и

$$
2 \operatorname{Br} \mathfrak{g m}=\sigma(Y)-U \circ U \bmod 16,
$$

əде $U \circ U-$ нормальное число Эйлера поверхности $U$ в $Y$.

Доказательство в случае, когда $Y$ односвязно (или в более обшем случае, когда $\left.K=H_{1}(U)\right)$, см. в [46], самый обший случай см., например, в [29]. Рохлин рассматривал случай, когда характеристическая поверхность ориентируема и использовал Arf-инвариант: в этом случае 4 Arf $=\mathrm{Br}$.

ЗАмечАнИЕ. Имеется другое построение формы Рохлина-Гийю-Марена. Так как поверхность $U$ характеристическая, $Y \backslash U$ допускает спинорную структуру, которая не продолжается ни через одну компоненту поверхности $U$. Сужение этой структуры на край трубчатой окрестности поверхности $U$ индуцирует естественным образом $\mathrm{Pin}^{-}{ }^{-}$-структуру на $U$ (cp. [38]), которая определяет квадратичную форму $q$ на $H_{1}(U)$. Нетрудно проверить, что $q$ определена с точностью до слагаемых из $\operatorname{Im}\left[i^{*}: H^{1}(Y) \rightarrow\right.$ $\left.H^{1}(U)\right]$ и, следовательно, ее сужение на $K$ не зависит от выбора спинорной структуры; это сужение совпадает с $\mathfrak{g m}$. (Таким образом получается другое доказательство теоремы C.0.1, о котором сообшил нам С. Бейли: квадратичные формы, возникающие из $\mathrm{Pin}^{-}$-структур, изоморфны друг другу, так как имеют один и тот же инвариант Брауна (см. В.1.2); эти формы дают все квадратичные продолжения формы $\mathfrak{g m}$ с $K$ на $H_{1}(U)$ и потому, как показано в [9], $K$ информативно.)

\section{СПИСОК ЛИТЕРАТУРЫ}

[1] Akbulut S. On quotients of complex surfaces under complex conjugation // J. Reine Angew. Math. 1994. V. 447. P. 83-90.

[2] Арнольд В.И. О расположении овалов вешественных плоских алгебраических кривых, инволюциях четырехмерных гладких многообразий и арифметике целочисленных квадратичных форм // Функц. анализ и его прил. 1971. Т. 5. № 3. С. 1-9.

[3] Арнольд В.И.Индекс особой точки векторного поля, неравенства Петровского-Олейник и смешанные структуры Ходжа // Функц. анализ и его прил. 1978. Т. 12. №1. С. 1-14.

[4] Арнольд В.И. Разветвленное накрытие $\mathbb{C} P^{2} \rightarrow S^{4}$, гиперболичность и проективная топология // Сиб. матем. журн. 1988. Т. 29. № 5. С. 36-47.

[5] Arnold V.I. Topological content of the Maxwell theorem on multipole representation of spherical functions // Topol. Methods Nonlinear Anal. 1996. V. 7. № 2. P. 205-217.

[6] Арнольд В.И., Олейник О.А. Топология вещественных алгебраических многообразий // Вестник МГУ. Сер. 1, матем. мех. 1979. №6. С. 7-17.

[7] Atiyah M.F. K-theory and reality // Quart. J. Math. Oxford Ser. (2). 1966. V. 17. P. $367-386$.

[8] Atiyah M.F., Dupont J. L. Vector fields with finite singularities // Acta Math. 1972. V. 128. P. $1-40$. 
[9] Bailly C., Vdovina A. Sous-espaces déterminant l'invariant d'Arf et un Théorème de Rokhlin sur la signature // C. R. Acad. Sci. Paris Sér. I Math. 2000. V. 330. № 3. P. 221-223.

[10] Barth W., Peters C., van de Ven A. Compact complex surfaces. Ergeb. Math. Grenzgeb. (3). Berlin: Springer-Verlag, 1984.

[11] Bihan F. Constructions combinatoires de surfaces algébriques réelles. Thèse. Rennes: Univ. Rennes I, 1998.

[12] Borel A., Haefliger A. La classe d'homologie fondamentale d'un espace analytique // Bull. Soc. Math. France. 1961. V. 89. P. 461-513.

[13] Бредон Г. Е. Введение в теорию компактных групп преобразований. М.: Наука, 1980.

[14] Cerf J. Sur les difféomorphismes de la sphére de dimension trois, $\Gamma_{4}=0 / /$ Lecture Notes in Math. 1968. V. 53.

[15] Ciliberto C., Pedrini C. Annibale Comessatti and real algebraic geometry // Rend. Circ. Mat. Palermo (2) Suppl. 1994. № 36. P. 71-102.

[16] Чжень Шэн-шень. Комплексные многообразия. М.: ИЛ, 1961.

[17] Comessatti A. Sulla connessione delle superficie algebriche reale // Verh. Internat. Math. Kongr. Zürich. 1932. V. 2. P. 169.

[18] Comessatti A. Fondamenti per la geometria sopra le superficie razionali dal punto di vista reale // Math. Ann. 1912. V. 43. P. 1-72.

[19] Comessatti A. Sur la connessione delle superficie razionali reale // Annali di Matematica. 1914. V. 23. P. 215-285

[20] Comessatti A. Reele Fragen in der algebraischen Geometrie // Jahresber. Deutsch. Math.-Verein. 1932. V. 41. P. 107-134.

[21] Conner P. E., Miller E. Y. Equivariant self-intersection // Preprint, 1979.

[22] Данилов В.И., Хованский А. Г. Многогранники Ньютона и алгоритм вычисления чисел Ходжа-Делиня // Изв. АН СССР. Сер. матем. 1986. Т. 50. № 5. С. 925-945.

[23] Degtyarev A. Stiefel orientations on a real algebraic variety // Lecture Notes in Math. 1992. V. 1524. P. 205-220.

[24] Degtyarev A. Cohomology approach to killing structures on Steenrod bundles // Adv. Soviet Math. 1994. V. 18. P. 1-22.

[25] Degtyarev A. On the Pontrjagin-Viro form // Adv. Math. Sci. Appl. Rokhlin's Memorial volume (to appear).

[26] Degtyarev A., Kharlamov V. Halves of a real Enriques surface // Comment. Math. Helv. 1996. V. 71. P. 628-663.

[27] Degtyarev A., Kharlamov V. Topological classification of real Enriques surfaces // Topology. 1996. V. 35. № 3. P. 711-729.

[28] Дегтярев А. И., Звонилов В. И. Жесткая изотопическая классификация вешественных алгебраических кривых бистепени $(3,3)$ на квадриках // Матем. заметки. 1999. Т. 66. №6. C. $810-815$.

[29] Degtyarev A., Itenberg I., Kharlamov V. Real Enriques Surfaces // Lecture Notes in Math. (to appear).

[30] Donaldson S. Yang-Mills invariants of smooth four-manifolds // Geometry of Low-Dimensional Manifolds. V. 1. Gauge Theory and Algebraic Surfaces. Cambridge: Cambridge Univ. Press, 1990. P. 5-40.

[31] Edmonds A. L. Orientability of fixed point sets // Proc. Amer. Math. Soc. 1981. V. 82. № 1. P. 120-124.

[32] Элиашберг Я. М., Харламов В. М. О числе комплексных точек вещественной поверхности в комплексной // Труды Ленинградской международной топологической конференциил. Л.: Наука, 1983. С. 143-148.

[33] Фидлер Т. Пучки прямых и топология вещественных алгебраических кривых // Изв. АН СССР. Сер. матем. 1982. Т. 46. №4. С. 853-863.

[34] Фидлер Т. Новые сравнения в топологии вещественных плоских алгебраических кривых // Докл. АН СССР. 1983. Т. 270. №1. С. 56-58.

[35] Фидлер Т. Дополнительные неравенства в топологии вещественных плоских алгебраических кривых // Изв. АН СССР. Сер. матем. 1985. Т. 49. № 4. С. 874-883. 
[36] Finashin S. Rokhlin conjecture and quotients of complex surfaces by complex conjugation // J. Reine Angew. Math. 1996. V. 481. P. 55-71.

[37] Finashin S. Decomposability of quotients by complex conjugation for rational and Enriques surfaces // Topology Appl. 1997. V. 79. № 2. P. 121-128.

[38] Финашин С. М. Инвариант относительно $\mathrm{Pin}^{-}$кобордизма и обобщение сигнатурного сравнения Рохлина // Алгебра и анализ. 1990. Т. 2. №4. С. 242-250.

[39] Finashin S., Shustin E. On imaginary plane curves and Spin-quotients of complex surfaces by complex conjugation // Trans. Amer. Math. Soc. 1997. V. 180. P. 93-101.

[40] Гудков Д. А. Построение новой серии $M$-кривых // Докл. АН СССР. 1971. Т. 200. №6. С. $1269-1272$.

[41] Гудков Д. А. Топология вещественных проективных алгебраических многообразий // УМН. 1974. Т. 29. № 4. С. 3-79.

[42] Гудков Д. А. О топологии алгебраических кривых на гиперболоиде // УМН. 1979. T. 34. № 6. C. 26-32.

[43] Гудков Д.А., Шустин Е.И. Классификация неособых кривых восьмого порядка на эллипсоиде // Методы качественной теории дифференциальных уравнений. Горький: ГГУ, 1980. С. 104-107.

[44] Гудков Д. А., Усачев А. К. Неособые кривые младших порядков на гиперболоиде // Методы качественной теории дифференциальных уравнений. Горький: ГГУ, 1980. C. $96-103$.

[45] Гудков Д. А., Уткин Г. А. Топология кривых 6-го порядка и поверхностей 4-го порядка // Уч. зап. Горьк. ун-та. 1969. Т. 87. С. 3-213.

[46] Guillou L., Marin A. Une extension d'un théorème de Rohlin sur la signature // C. R. Acad. Sci. Paris. 1977. V. 285. P. А95-A98; // рус. перев. в кн.: В поисках утраченной топологии. Под ред. Л. Гийу, А. Марена. М.: Мир, 1989. С. 110-134.

[47] Хартсхорн Р. Алгебраическая геометрия. М.: Мир, 1981.

[48] Hirzebruch F. Über vierdimensionale Riemannsche Flächen mehrdeufiger analitischer Functionen von zwei komplexen Veränderlichen // Math. Ann. 1953. V. 126. № 1. P. 1-22.

[49] Хирцебрух Ф. Топологические методы в алгебраической геометрии. М.: Мир, 1973.

[50] Horikawa E. On deformations of quintic surfaces // Invent. Math. 1975. V. 31. P. 43-85.

[51] Itenberg I., Kharlamov V. Towards the maximal number of components of a nonsingular surface of degree 5 in $\mathbb{R P}^{3} / /$ Amer. Math. Soc. Transl. Ser. 2. 1996. V. 173. P. 111-118.

[52] Itenberg I., Viro O. Ya. Patchworking algebraic curves disproves the Ragsdale conjecture // Math. Intelligencer. 1996. V. 18. № 4. P. 19-28.

[53] Itenberg I., Viro O. Ya. Maximal real algebraic hypersurfaces of projective space // Preprint.

[54] Kalinin I. Когомологические характеристики вещественных проективных гиперповерхностей // Алгебра и анализ. 1991. Т. 3. № 2. С. 91-110.

[55] Харламов В. М. Максимальное число компонент поверхности 4-й степени в $\mathbb{R P}^{3} / /$ Функц. анализ и его прил. 1972. Т. 6. № 4. С. 101.

[56] Харламов В. М. Обобщенное неравенство Петровского // Функц. анализ и его прил. 1974. Т. 8. №2. С. 50-56.

[57] Харламов В.М. Топологические типы неособых поверхностей степени 4 в $\mathbb{R} \mathbb{P}^{3} / /$ Функц. анализ и его прил. 1976. Т. 10. № 4. С. 55-68.

[58] Харламов В. М. Обобщенное неравенство Петровского. II // Функц. анализ и его прил. 1975. Т. 9. № 3. С. 93-94.

[59] Харламов В. М. Дополнительные сравнения для эйлеровой характеристики четномерных вещественных алгебраических многообразий // Функц. анализ и его прил. 1975. Т. 9. № 2. C. $51-60$.

[60] Харламов В. М. Изотопические типы неособых поверхностей степени 4 в $\mathbb{R P}^{3} / /$ Функц. анализ и его прил. 1978. Т. 12. № 1. С. 86-87.

[61] Харламов В. М. К класификации неособых поверхностей степени 4 в $\mathbb{R P}^{3}$ относительн жестких изотопий // Функц. анализ и его прил. 1984. Т. 18. № 1. С. 49-56. 
[62] Kharlamov V. Nonamphicheiral surfaces of degree 4 in $\mathbb{R P}^{3} / /$ Lecture Notes in Math. 1988. V. 1346. P. $349-356$.

[63] Харламов В.M. О числе компонент $M$-поверхности степени 5 в $\mathbb{R P}^{3} / /$ Труды XVI советской алгебраической конференции. Л., 1981. С. 353-354.

[64] Kharlamov V. Estimates of Betti numbers in topology of real algebraic surfaces // Proc. Internat. Topological Conference. Oberwolfach, 1987. P. 12-13.

[65] Хованский А.Г. Индекс полиномиального векторного поля // Функц. анализ и его прил. 1979. Т. 13. № 1. С. 49-58.

[66] Хованский А.Г. Краевые индексы полиномиальных 1-форорм с однородными компонентами // Алгебра и анализ. 1998. Т. 10. № 3. С. 193-222.

[67] Klein F. Über Flächen dritter Ordnung // Math. Ann. 1873. V. 6. P. 551-581.

[68] Kodaira K., Spencer D. C. Groups of complex line bundles over compact Kähler varieties. Divisor class groups on algebraic varieties // Proc. Nat. Acad. Sci. U.S.A. 1953. V. 39. P. 868-877.

[69] Korchagin A. Classification of real algebraic curves on toric varieties, Preliminary report // 911-th AMS Meeting, Program. Louisiana State Univ., Baton Rouge, Apr. 19-21, 1996.

[70] Краснов В.А. Неравенства Харнака-Тома для отображений вещественных алгебраических многообразий // Изв. АН СССР. Сер. матем. 1983. Т. 47. № 2. С. 268-297.

[71] Краснов В.А. Об эквивариантных когомологиях Гротендика вещественного алгебраического многообразия и их приложениях // Изв. РАН. Сер. матем. 1994. Т. 58. № 3. C. $36-52$.

[72] Kuiper N. The quotient space of $\mathbb{C} P^{2}$ by complex conjugation is the 4-sphere // Math. Ann. 1974. V. 208. P. 175-177.

[73] Lai Hon-Fei. Characteristic classes of real manifolds immersed in complex manifolds // Trans. Amer. Math. Soc. 1972. V. 172. P. 1-33.

[74] Laudenbach F., Poenaru V. A note on four-dimensional handlebodies // Bull. Soc. Math. France. 1972. V. 100. P. 337-344.

[75] Letizia M. Quotients by complex conjugation of nonsingular quadrics and cubics in $\mathbb{C} P^{3}$ defined over $\mathbb{R}$ // Pacific J. Math. 1984. V. 110. № 2. P. 307-314.

[76] Марен А. $\mathbb{C} P^{2} / \sigma$ или Кейпер и Масси в стране коник // В поисках утраченной топологии. Под ред. А. Гийу, А. Марена. М.: Мир, 1989. С. 149-161.

[77] Марен А. Несколько замечаний о вещественных плоских алгебраических кривых // В поисках утраченной топологии. Под ред. А. Гийу, А. Марена. М.: Мир, 1989. С. 162-172.

[78] Massey W. The quotient space of the complex projective plane under conjugation is a 4-sphere // Geom. Dedicata. 1973. V. 2. P. 371-374.

[79] Matsuoka S. Nonsingular algebraic curves in $\mathbb{R P}^{1} \times \mathbb{R P}^{1} / /$ Trans. Amer. Math. Soc. 1991. V. 324. № 1. P. 87-107.

[80] Matsuoka S. Congruences for $M$ - and $(M-1)$-curves with odd branches on a hyperboloid // Bull. London Math. Soc. 1992. V. 24. P. 61-67.

[81] Mikhalkin G. The complex separation of real surfaces and extensions of Rokhlin congruence // Invent. Math. 1994. V. 118. P. 197-222.

[82] Mikhalkin G. Adjunction inequality for real algebraic curves // Math. Res. Lett. 1997. V. 4. №1. P. 45-52.

[83] Mikhalkin G. Congruences for real algebraic curves on an ellipsoid // Adv. Soviet Math. 1994. V. 18. P. 223-233.

[84] Mikhalkin G. Topology of curves of degree 6 on cubic surfaces in $\mathbb{R P}^{3} / / \mathrm{J}$. Algebraic Geom. 1998. V. 7. № 2. P. 219-237.

[85] Милнор Дж., Хьюзмоллер Д. Симметрические билинейные формы. М.: Наука, 1979.

[86] Милнор Дж., Сташеф Дж. Характеристические классы. М.: Мир, 1979.

[87] Натанзон С. М. Клейновы поверхности // УМН. 1990. Т. 45. №6. С. 47-90.

[88] Никулин В.В.Целочисленные симметрические билинейные формы и некоторые их геометрические приложения // Изв. АН СССР. Сер. матем. 1979. Т. 43. № 1. С. 111-177. 
[89] Никулин В.В.Инволюции целочисленных квадратичных форм и их приложения к вещественной алгебраической геометрии // Изв. АН СССР. Сер. матем. 1983. Т. 47. № 1. C. $109-188$.

[90] Ochanine S. Signature modulo 16, invariants de Kervaire generalisés et nombres charactéristiques dans la $K$-théorie réelle // Mém. Soc. Math. France (N.S.). 1981. № 5. P. 1-142.

[91] Олейник О.А. О топологии действительных алгебраических кривых на алгебраической поверхности // Матем. сб. 1951. Т. 29. № 1. С. 133-156.

[92] Orevkov S. Link theory and oval arrangements of real algebraic curves // Topology. 1999. V. 38. №4. P. 779-810.

[93] Orevkov S. Riemann existence theorem and construction of real algebraic curves (to appear).

[94] Petrovskiy I. G. On the topology of real plane algebraic curves // Ann. Math. 1938. V. 39. № 1. P. 189-209.

[95] Петровский И.Г., Олейник О.А. О топологии действительных алгебраических поверхностей // Изв. АН СССР. Сер. матем. 1949. Т. 67. № 13. С. 389-402.

[96] Risler J.-J. Construction d'hypersurfaces réelles (d'après Viro) // Astérisque. 1993. V. 216. P. $69-86$.

[97] Рохлин В.А.Доказательтсво гипотезы Гудкова // Функц. анализ и его прил. 1972. Т. 6. № 2. C. $62-64$.

[98] Рохлин В. А. Комплексные ориентации вещественных алгебраических кривых // Функц. анализ и его прил. 1974. Т. 8. № 4. С. 71-75.

[99] Рохлин В.А. Комплексные топологические характеристики вещественных алгебраических кривых // УМН. 1978. Т. 33. №5. С. 77-89.

[100] Рохлин В. А. Сравнения по модулю 16 в шестнадцатой проблеме Гильберта // Функц. анализ и его прил. 1972. Т. 6. № 4. С. 58-64.

[101] Рохлин В.А.Сравнения по модулю 16 в шестнадцатой проблеме Гильберта. II // Функц. анализ и его прил. 1973. Т. 7. № 2. С. 91-92.

[102] Рохлин В.А. Новые неравенства в топологии вещественных плоских алгебраических кривых // Функц. анализ и его прил. 1980. Т. 14. № 1. С. 37-43.

[103] Saito S. (formerly S. Matsuoka). Classification of involutions of lattices with conditions and real algebraic curves on a hyperboloid // Sūrikaisekikenkyūsho Kōkyūroku. 1977. V. 1006. P. 1-21.

[104] Scharlau W. Quadratic and Hermitian Forms. Berlin: Springer-Verlag, 1985. (Grundlehren Math. Wiss. V. 270.)

[105] Schläfli L. On the distribution of surfaces of the third order into species, in reference to the absence or presence of singular points, and the reality of their lines // Philos. Trans. Roy. Soc. London. 1863. V. 153. P. 195-241.

[106] Segre B. The Non-Singular Cubic Surfaces. Oxford: Oxford Univ. Press, 1942.

[107] Silhol R. Real Algebraic Surfaces. Berlin: Springer-Verlag, 1989. (Lecture Notes in Math. V. 1392.)

[108] Steenbrink J. Intersection form for quasi-homogeneous singularities // Compositio Math. 1977. V. 34. № 2. P. 211-223.

[109] Steenrod N.E., Epstein D. B. A. Cohomology Operations. Princeton: Princeton Univ. Press, 1962.

[110] Szabó Z. Simply-connected irreducible 4-manifolds with no symplectic structure // Invent. Math. 1998. V. 132. P. 457-466.

[111] Thom R. Une théorie intrinsèque des puissances de Steenrod // Colloque de Topologie de Strasbourg (1951) 1952. Exposé N. 6.

[112] Thom R. Espaces fibrés en sphères et carrés de Steenrod // Ann. Sci. École Norm. Sup. 1952. V. 3. № 2. P. 109-182.

[113] Thom R. Sur l'homologie des variétés algébriques réelles // Differ. Combinat. Topology: A symposium in honour of Marston Morse. Princeton: Princeton Univ. Press, 1965. P. 255-265.

[114] Варченко А. Н. Теоремы топологической эквисингулярности семейств алгебраических многообразий и полиномиальных отображений // Изв. АН СССР. Сер. матем. 1972. Т. 36. № 5. C. 957-1019. 
[115] Варченко А. Н. Асимптотическая смешанная структура Ходжа в исчезаюших когомологиях // Изв. АН СССР. Сер. матем. 1981. Т. 45. № 3. С. 540-591.

[116] Варченко А. Н. О локальном вычете и форме пересечений в исчезающих когомологиях // Изв. АН СССР. Сер. матем. 1985. Т. 49. № 1. С. 32-54.

[117] Виро О.Я. Плоские вешественные алгебраические кривые: построения с контролируемой топологией // Алгебра и анализ. 1989. Т. 1. № 5. С. 1-73.

[118] Виро О.Я. Построение $M$-поверхностей // Функц. анализ и его прил. 1979. Т. 13. № 3. C. $71-72$.

[119] Виро О.Я. Успехи в топологии вещественных алгебраических многообразий за последние шесть лет // УМН. 1986. Т. 41. №3. С. 45-67.

[120] Viro O. Ya. Complex orientations of real algebraic surfaces // Adv. Soviet Math. 1994. V. 18. P. 261-284.

[121] Wall C. T. C. Quadratic form in finite groups and related topics // Topology. 1964. V. 2. P. 281-298.

[122] Wallace A. Linear sections of algebraic varieties // Indiana Univ. Math. J. 1971. V. 20. P. 1153-1162.

[123] Wang Sh. A vanishing theorem for Seiberg-Witten invariants // Math. Res. Lett. 1995. V. 2. №3. P. 305-310.

[124] Welschinger J.-Y. Orientations complexes des $J$-courbes réelles // Prépublications de l'Univ. Louis Pasteur, Strasbourg, 1999.

[125] Welschinger J.-Y. J-courbes réelles à nids profonds sur les surfaces réglées // Prépublications de l'Univ. Louis Pasteur, Strasbourg, 1999.

[126] Wilson G. Hilbert's sixteenth problem // Topology. 1978. V. 17. P. 53-73.

[127] Zeuthen H. G. Etudes des propriétés de situation des surfaces cubiques // Math. Ann. 1875. V. 8. P. $1-30$.

[128] Звонилов В.И. Неравенства Харламова и неравенства Петровского-Олейник // Функц. анализ и его прил. 1975. Т. 9. № 2. С. 69-70.

[129] Звонилов В.И.Комплексные топологические характеристики вещественных алгебраических кривых на поверхности // Функц. анализ и его прил. 1982. Т. 16. № 3. С. 56-57.

[130] Звонилов В.И.Комплексные топологические инварианты вещественных алгебраических кривых на гиперболоиде и эллипсоиде // Алгебра и анализ. 1991. Т. 3. №5. С. 88-108.

[131] Zvonilov V. I. Stratified spaces of real algebraic curves of bidegree $(m, 1)$ and $(m, 2)$ on a hyperboloid // Amer. Math. Soc. Transl. Ser. 2. 1996. V. 173. P. 253-264.

Bilkent University, Ankara, Turkey;

Поступила в редакцию

Institut de Recherche Mathématique Avancée

09.12.1999

Université Louis Pasteur et CNRS

7 rue René-Descartes

67084, Strasbourg, France

E-mail: degt@fen.bilkent.edu.edu, kharlam@math.u-strasbg.fr 